\title{
Resistance to microtubule-stabilising agents following point mutation of human $\beta I$-tubulin
}

by

Matthew R. Rowe

2015

A thesis submitted to Victoria University of Wellington in fulfilment of the requirements for the degree of Master of Biotechnology

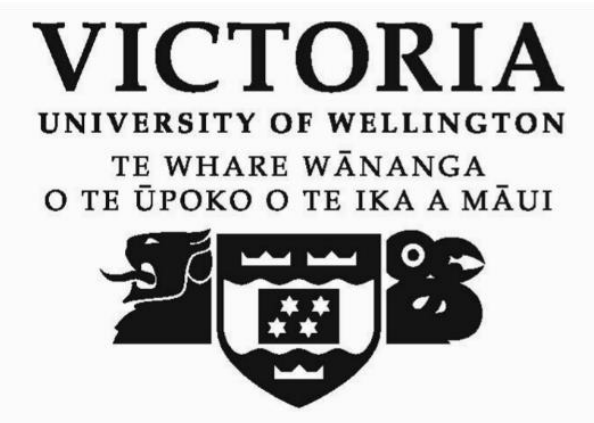





\begin{abstract}
Marine environments represent a rich source of bioactive secondary metabolites that may be harnessed for use in a therapeutic context. Two novel compounds, peloruside A and laulimalide, isolated from the marine sponges Mycale hentsheli and Cacospongia mycofijiensis, respectively, both demonstrate useful pharmacological properties in mammalian cells. These compounds share major similarities with microtubule-stabilising agents. Like other agents in this class, peloruside $\mathrm{A}$ and laulimalide bind to the $\beta$-tubulin subunit of microtubules, the primary cytoskeletal element of eukaryotic cells. These compounds enhance polymerisation dynamics between ternary microtubule structures and severely hinder necessary cytoskeletal rearrangements within the cell.
\end{abstract}

Over the course of a patient's treatment, cancerous cells may develop multi-drug resistance phenotypes. P-glycoprotein drug efflux pumps play a major role in the development of therapy resistance in many cancers, as the current generation microtubule-stabilising agents are easily removed from diseased cells by upregulated efflux mechanisms. Unlike agents already in clinical application, both peloruside A and laulimalide are poor substrates for removal by these mechanisms, making them and their synthetic derivatives interesting as potential treatments for drug-resistant tumours.

Peloruside A and laulimalide exhibit potent nanomolar anti-mitotic activities in vitro and arrest cell cycle progression in $\mathrm{G}_{2} / \mathrm{M}$ phase, leading to cell death - a characteristic mode of action among microtubule-stabilising agents. Unlike all known agents in this class, peloruside $\mathrm{A}$ and laulimalide share a secondary, unique binding region in $\beta$-tubulin. In the past decade our understanding of this region has developed, revealing a second, unique mechanism for stabilisation of microtubules. 
Using mammalian cells to model physiological tubulin, the present study investigates the predicted role of aspartic acid 297 of human $\beta \mathrm{I}$-tubulin in the binding association of both peloruside A and laulimalide. This particular amino acid is predicted to hydrogen bond with both compounds, contributing to their activity as stabilisers.

It was revealed that the introduction of a point mutation in D297 resulted in a small but highly consistent resistance phenotype to both compounds, but not to microtubulestabilising agents that bind to the traditional, taxoid site on $\beta$-tubulin. It was concluded that aspartic acid 297 is likely to be one of the amino acids directly involved in the binding association of peloruside $\mathrm{A}$ and laulimalide to $\beta$-tubulin, contributing partial compound stabilisation. The rational synthesis of future analogues may benefit from these findings in the design of molecules with enhanced interactions at this particular amino acid residue. 


\section{Acknowledgements}

It brings me great joy to begin this thesis with the foremost acknowledgement of my outstanding supervisor, Prof John H. Miller, to whom I owe countless thanks for his constant guidance, reassurance, experience and most of all patience as I have progressed through these studies. Without your endless support through some difficult patches I would not be here, writing this page and for this I am truly grateful.

I am pleased to acknowledge Assoc. Prof Paul Teesdale-Spittle, for valued input into many facets of this research design, in addition to his respected advice and discussion across all aspects of this investigation.

To Dr. Jessica J. Field, to whom I owe great recognition for sharing her knowledge in many of the practical aspects of this research. Thank you for showing me the ropes and keeping everything running in the JHM lab while I was getting up to speed.

It is with warm thoughts and appreciation that I recognize Dr. Melanie J. McConnell, for whom I hold great respect as both a mentor and friend. Your depth of knowledge and youthful enthusiasm for all things biology has influenced me greatly during the course of these investigations. Please continue to inspire many more students similarly in future.

To my many friends and colleagues within the SBS, particularly those within the Centre for Biodiscovery. I thank the excellent team of people that make up this department. Students, some more than others; thank you for putting up with my never ending curiosity towards each of your projects. You all know how much I love to discuss science, but hopefully in return you also gained from my banter. There are too many to name here individually but you each know what your friendships mean to me.

Thanks to Assoc. Prof Peter Northcote and Jonathan Singh for making natural products available for use in these studies. I hope this investigation represents a small yet valuable contribution to the work of the natural products group here at Victoria University.

Finally, I would like to put forward my deepest gratitude's to my family. Paula, Richard and Anna - Without your support and encouragement emotionally, financially and in many more ways than I have words, I wouldn't have been able to do some of the pretty cool stuff that's here inside this thesis. 



\section{TABLE OF CONTENTS}

ABSTRACT

ACKNOWLEDGEMENTS III

TABLE OF FIGURES VIII

LIST OF TABLES IX

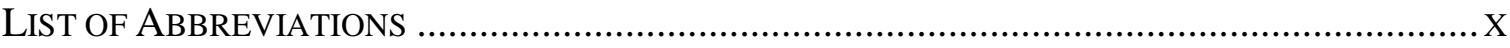

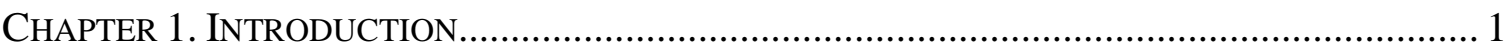

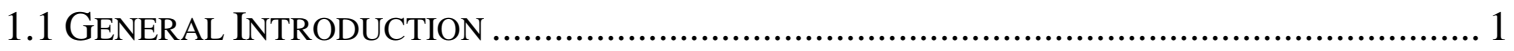

1.1.1 Bioactive compounds sourced from nature ................................................................... 1

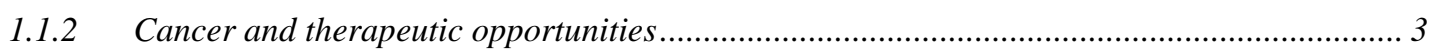

1.1.3 Microtubules as targets in chemotherapeutic approaches to cancer treatment ....................... 5

1.1.4 Introduction to human tubulin genes............................................................................. 9

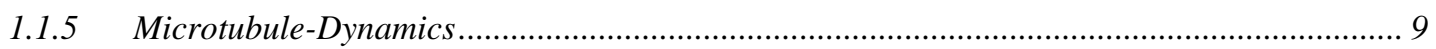

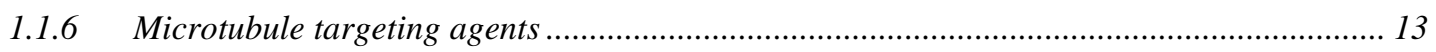

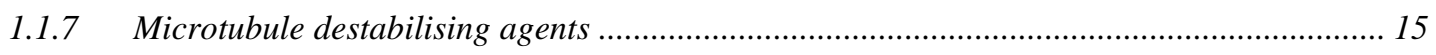

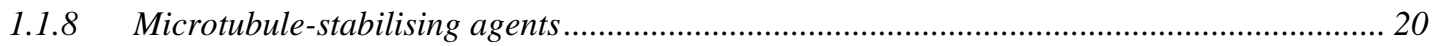

1.1.9 Microtubule-stabilising agents of marine origin .............................................................. 24

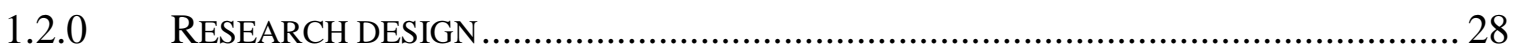

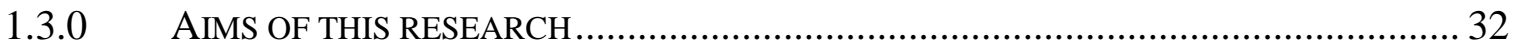

1.3.1 Examine the functional role of the D297 amino acid residue of human BI-tubulin in the predicted binding association of Pel A and Lau.

1.3.2 Determine optimum transfection parameters for mutant $\beta I$-tubulin expression in a mammalian cell line.

1.3.3 Examine the integration of exogenous mutant tubulin subunits into polymerised microtubules

1.3.4 Examine the concentration-response of D297I mutant transfected cells in response to drugs targeting the taxane region of $\beta$-tubulin

CHAPTER 2. EXOGENOUS HUMAN B1-TUBULINS AS TOOLS FOR INVESTIGATIONS OF

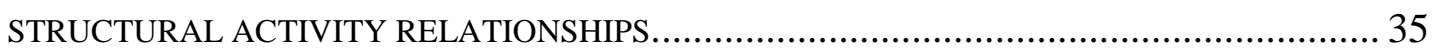

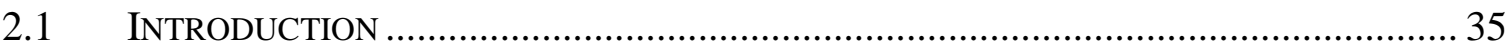

2.1.1 MAMMALIAN CELL MODELS FOR THE STUDY OF DRUG-PROTEIN INTERACTIONS . 35

2.1.2 INTRODUCTION TO TRANSFECTION AND EXOGENOUS GENE EXPRESSION.............. 36

2.1.3 PREDiCTED INTERACTIONS OF PEL A/LAU WITH BI-TUbULIN.............................. 37

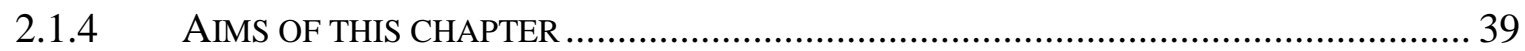

A) Optimise transfection parameters to maximise tGFP-D297I- $\beta I$-tubulin and $t G F P$ - $\beta I$-tubulin expression throughout a mammalian cell population 
B) Quantify exogenous expression of mutant $\beta I$-tubulins in the transfected cell population of paired concentration-response experiments.....

C) Examine the morphology of mutant tubulin-containing subunits within polymerised microtubules

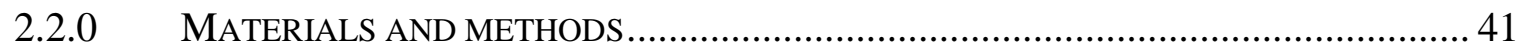

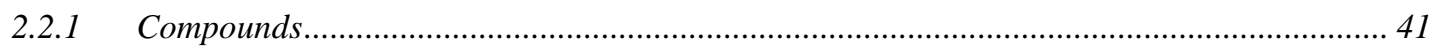

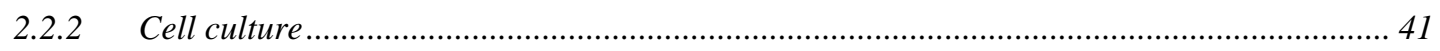

2.2.3 Mammalian expression vector design and preparation ................................................. 42

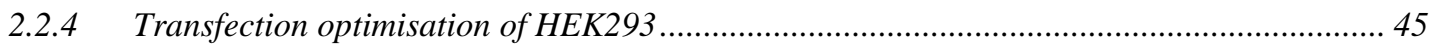

2.2.5 Transfection of HEK293 for experimental applications.................................................... 45

2.2.6 Confirmation of transfection by fluorescence microscopy .................................................. 46

2.2.7 Confirmation of exogenous tubulin expression by western blotting ...................................... 47

2.2.8 Microtubule morphology and confirmation of mutant-tubulin expression by live cell confocal

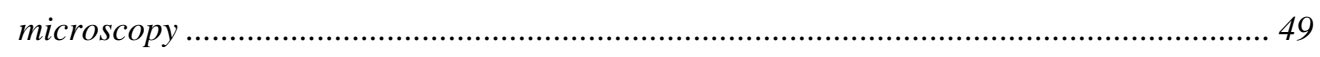

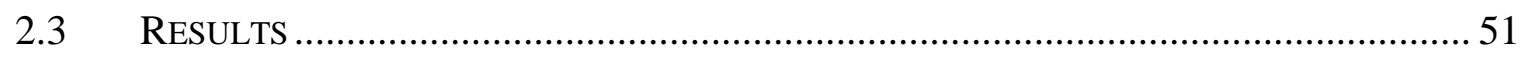

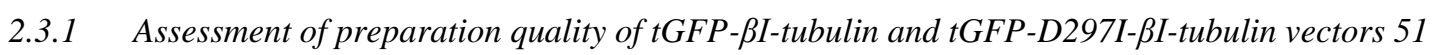

2.3.2 Optimisation of transfection in HEK293 cells............................................................. 53

2.3.3 Confirmation of HEK293 transfection by fluorescence microscopy ................................. 56

2.3.4 Transfection efficiencies in 1 A9 cells ....................................................................... 57

2.3.6 Confirmation of exogenous tubulin expression by Western blotting:.................................. 59

2.3.5 Microtubule morphology and conformation of mutant-tubulin expression using live cell

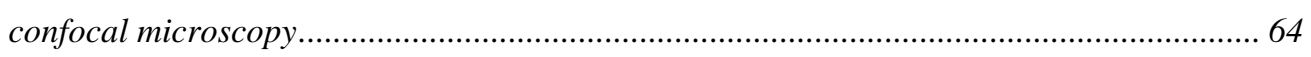

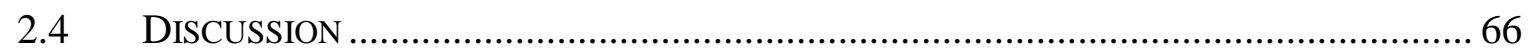

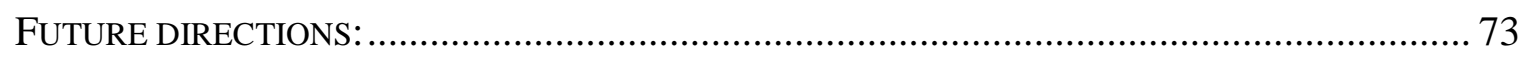

CHAPTER 3. INVESTIGATIONS INTO THE DIFFERENTIAL DRUG SENSITIVITY OF MUTANT TGFP-D297I-BI-TUBULIN IN HEK293 CELLS .................................. 76

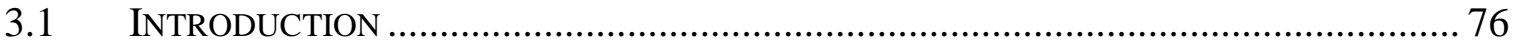

3.1.1 Introduction to cytotoxicity screening and cell proliferation assays .................................... 76

3.1.2 Introduction to in situ tubulin dynamics........................................................................... 77

3.2.0 AIMS OF THIS CHAPTER......................................................................... 79

3.2.1 Evaluate the concentration-response of cells with an expressed D297 point mutant BI-tubulin population in response to Pel A and Lau ....................................................................... 79

3.2.2 Examine the concentration-response of transfected cells treated with PTX or IXA to determine the effect of the point mutations presence, independent of direct ligand binding to the D297I substitution.

3.2.3 Examine microtubule polymerisation states for D297I- $\beta$ I-tubulin point mutation transfected cells in response to $\mathrm{Pel} A$ 
3.3.1 Cellular proliferation of D297I mutant $\beta 1$-tubulin HEK293 by MTT in response to microtubule-stabilisers

3.3.2 In situ polymerisation of $\beta 1$-tubulin containing D297I mutant isotype subunits in response to

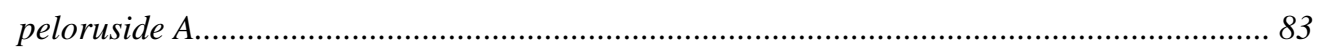

3.3.3 Secondary assessment of D297I mutant $\beta 1$-tubulin presence in HEK293 cells .................... 85

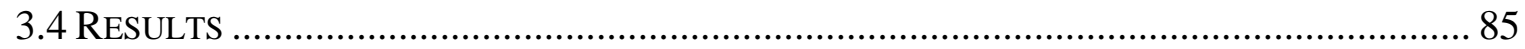

3.4.1 Assessment of transfection impact on HEK293 in response to PTX and IXA........................ 85

3.4.2 Assessment of transfection impact on HEK293 in response to Pel A or Lau ........................ 91

3.4.3 Effect of Pel A on in situ polymerisation of $\beta 1$-tubulin containing D297I mutant subunits .. 97

3.4.4 Secondary re-assessment of D297I mutant $\beta 1$-tubulin presence in HEK293 ...................... 100

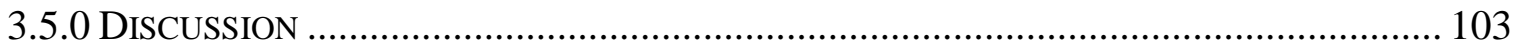

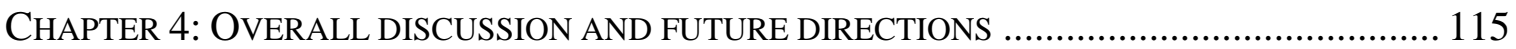

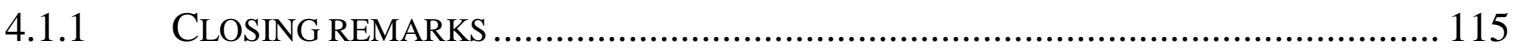

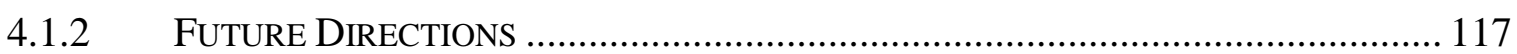

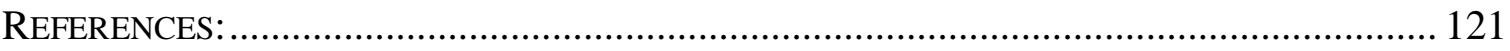

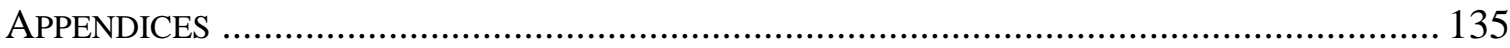

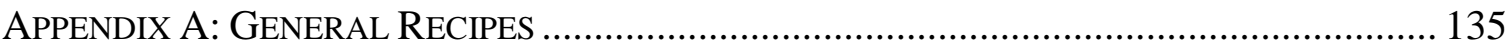




\section{Table of Figures}

\section{Chapter One}

$\begin{array}{ll}\text { Figure 1.1.1 Linearized peptide sequence of } \beta \text {-tubulin } & 7\end{array}$

$\begin{array}{lr}\text { Figure 1.1.2 Microtubule polymer-assembly } & 8\end{array}$

Figure 1.1.3 Microtubule dynamics and macropolymer structure $\quad 11$

$\begin{array}{ll}\text { Figure 1.1.4 The colchicine binding region } & 17\end{array}$

$\begin{array}{ll}\text { Figure 1.1.5 The vinca domain binding region } & 19\end{array}$

Figure 1.1.6 The taxane binding region $\quad 22$

Figure 1.1.7 Chemical structures of novel marine products Pel A and Lau 25

Figure 1.1.8 The binding region of Pel A and Lau 30

\section{Chapter Two}

Figure 2.1.1 Binding interactions of Pel A and Lau 37

Figure 2.2.1 Cloning schematic for mutation plasmid. $\quad 44$

Figure 2.3.1 Agarose gel electrophoresis of tGFP- $\beta 1$-tubulin plasmid. 52

Figure 2.3.2 Agarose gel electrophoresis of tGFP-D297I-ß1-tubulin plasmid. 53

Figure 2.3.3 Assessment of transfection optimisations in HEK293 by fluorescence 55 microscopy

Figure 2.3.4 Further assessment of transfection optimisations in HEK293 by fluorescence 56 microscopy

Figure 2.3.5 Quantification of transfection efficiencies by fluorescence microscopy 57

Figure 2.3.6 1A9 cells transfected with tGFP-D297I- $\beta 1$-tubulin expression vector $\quad 58$

Figure 2.3.7 $\beta I$-tubulin profile of 1 A9 cells samples at $48 \mathrm{~h}$ and $144 \mathrm{~h}$ post-transfection $\quad 60$

Figure 2.3.8 Detection by antibodies targeting the $C$ - $v s-N$ terminus 61

Figure 2.3.9 Mutant expression in HEK293 cells 63

Figure 2.3.10 Live cell confocal microscopy $\quad 65$ 


\section{Chapter Three}

Figure 3.4.1 Concentration-response for mock control transfected HEK293 treated with 87 $P T X$ and IXA

Figure 3.4.2 Concentration-response for $t G F P-\beta I$ transfected HEK293 cells treated with 88 PTX and IXA

Figure 3.4.3 Concentration-response for $t G F P-D 297-\beta I$ mutant transfected HEK293 cells 89 with PTX and IXA

Figure 3.4.4 Summary of IC $C_{50}$ data for PTX or IXA concentration-response treatments in 90 transfected HEK293 compared to mock transfected cells.

Figure 3.4.5 Concentration-response for mock transfected HEK293 cells treated with Pel 92 $A$ and Lau

Figure 3.4.6 Concentration-response for WT tGDP-ßI transfected HEK293 cells treated 93 with Pel A and Lau

Figure 3.4.7 Concentration-response for tGDP-D297I-ßI mutant transfected HEK293 94 cells treated with Pel A and Lau

Figure 3.4.8 Summary of IC 50 data for Pel A and Lau treatments of HEK293 cells

Figure 3.4.9 Representative in situ polymerisation experiment for HEK293 cells treated 99 with Pel A

Figure 3.4.10 In situ polymerisation of HEK293 cells

Figure 3.4.11 Secondary reassessment of tGFP presence in tGFP-D297I transfected 102 HEK293

\section{List of Tables}

Table 3.4.1 Summary IC $_{50}$ data of IXA and PTX treated HEK293 cells

Table 3.4.2 Summary IC $_{50 \text { data }}$ of Pel A and Lau treated HEK293 cells 


\section{List of Abbreviations}

1A9

APS

BCA

$\mathrm{bp}$

BSA

CEM

CHAPS

CMV

D297I

D297I- $\beta$ I-tubulin

DMEM:

DMF

DMSO

DNA

EDTA

EGTA

EM

E-site

$\mathrm{EtOH}$

FCS:

FDA

G418

gDNA

GDP

GFP

GTP

$\mathrm{HCl}$

HDX-MS

HEK 293

HPLC

IC
Subclone of the A2780 human ovarian carcinoma cell line

Ammonium persulfate

Bicinchoninic acid

Base pair

Bovine serum albumin

Cryo-electron microscopy

3-[(3-Cholamidopropyl) dimethylammonio]-1-propanesulfonate cytomegalovirus

Aspartic acid substitution for isoleucine at residue 297

$\beta$ I-tubulin with isoleucine subtitution mutation at residue 297

Dulbecco's modified eagle medium

$\mathrm{N}, \mathrm{N}$-dimethylformamide

Dimethyl sulfoxide

Deoxyribonucleic acid

Ethylenediaminetetraacetic acid

Ethylene glycol tetraacetic acid

Electron microscopy

Exchangeable nucleotide site

Ethanol

Fetal Calf Serum

US Food and Drug Administration

Geneticin

Genomic DNA

Guanosine 5'-diphosphate

Green fluorescent protein

Guanosine-5'-triphosphate

Hydrochloric acid

Hydrogen deuterium exchange mass spectrometry

Human embryonic kidney cell line

High-performance liquid chromatography

Inhibitory concentration 
ICAT

IXA

Lau

LB

M

MALDI-TOF

MAP

MDA

MDR

$\mathrm{MgCl}_{2}$

MS

MSA

MTA

MTT

$\mathrm{NaCl}$

$\mathrm{NIH}$

N-site

ORSM

PBS

PCR

Pel A

Pgp

PTX

PVDF

RIPA

RPMI-1640

SAR

SD

SDS

SDS-PAGE

SEM

SLD

T2R

TBS
Isotope-coded afinity tags

Ixabepilone (Ixempra®)

Laulimalide

Lysogeny broth

Mitosis

Matrix assisted laser desorption ionisation-time of flight

Microtubule-associated proteins

Microtubule-destabilising agent

Multiple-drug resistance

Magnesium Chloride

Mass spectrometry

Microtubule-stabilising agent

Microtubule-targeting agent

2-(4,5-dimethyl-2thiazolyl)-3,5-diphenyl-2H-tetrazolium bromide

Sodium Chloride

National Institute of Health

Non-exchangeable nucleotide site

Optimem Reduced Serum Medium

Phosphate buffered saline

Polymerase chain reaction

Peloruside A

P-glycoprotein

Paclitaxel (Taxol®)

Polyvinylidene fluoride

Radioimmunoprecipitation assay

Roswell Park Memorial Institute medium 1640

Structure-activity relationships

Standard deviation

Sodium dodecyl sulfate

Sodium dodecyl sulfate polyacrylamide gel electrophoresis

Standard error of mean

Stathmin-like domain

Two tubulin heterodimers in complex with a SLD

Tris buffered saline 
TBST

TEMED

tGFP

TRIS

TTL

WHO

WT
Tris buffered saline with Tween-20

N,N,N',N'-tetramethylethylenediamine

Turbo GFP

Tris(hydroxymethyl)aminomethane

Tubulin tyrosine ligase

World Health Organisation

Wild type 



\section{Chapter 1. Introduction}

\subsection{General Introduction}

\subsubsection{Bioactive compounds sourced from nature}

Natural products are a particularly valuable source of bioactive compounds for use in a therapeutic context as the sheer diversity of biosynthetic processes found throughout nature has led to an unparalleled diversity in unique chemical structures (Harvey, Clark et al. 2010, Yue, Liu et al. 2010). These compounds often exhibit interesting pharmacological properties when extracted from raw biomass, particularly in the case of novel secondary metabolites sourced from marine environments (Faulkner, 2002). Through evolutionary development over millions of years, organisms produce secondary metabolites with exquisite bioactivities against protein targets in living systems.

Current techniques in organic chemistry allow the synthesis of vast libraries of novel organic structures; however, the majority of these compounds lack significant functional activity against biomolecular targets found within living systems (Leach and Hann 2000). This leaves synthetic compounds with somewhat limited potential in comparison to the exquisite bioactivity of natural products, provided by millions of years of evolutionary development against target proteins in living organisms. Active secondary metabolites are often produced by microbial organisms with symbiotic relationships to higher-level host organisms (Belarbi, Contreras Gómez et al. 2003) as chemical defence mechanisms against predation (Sipkema, Franssen et al. 2005) to support the host organism. Natural compounds that exhibit potent activities against protein targets within higher-order eukaryotic cells, such as those of a predatory species, are of great interest for drug discovery. The differences in biochemical processes between multicellular aquatic organisms like sponges and corals, microbes and terrestrial mammals represents an attractive niche for bioprospecting within marine environments, often leading to the discovery of compounds with useful therapeutic 
properties. These protein targets are often critical to the maintenance of regular cellular processes, thus the ability to disrupt normal biological activities in a predator is beneficial for both microbiota and the host-organism (Thakur and Müller 2004). Similarly, adaption of these compounds to interfere with an aberrant cellular process in a human diseases presents a convenient opportunity for exploitation for medicinal purposes. The ability to modulate mammalian biochemical pathways with small molecules has led to the development of many novel bioactive compounds in pharmaceutical applications (Buss and Butler 2004). Clinically, natural products and their semi-synthetic derivatives make up a large portion of front line therapeutic agents in the treatment of cancers (Newman and Cragg 2012), with many of the compounds interacting directly with targets in the cytoskeletal elements of eukaryotic cells. Compounds exhibiting bioactivity against critical protein targets, such as actin or tubulin, are of particular interest in the development of novel therapeutic agents. Secondary metabolites with these properties serve as an excellent structural scaffold for the design of variant analogues, tuned through synthetic chemistry to augment the initial bioactive properties of nature's template molecules. Modifications are made through a rational approach based on evidence provided by structure-activity relationship studies (SARs), leading to the eventual development of compounds with an enhanced therapeutic window. A combination of modern crystallographic techniques, chemoinformatics, ligand-based drug design and in silico computational approaches are continually improving our ability to identify highly-specific candidate molecules (Seddon, Lounnas et al. 2012). 


\subsubsection{Cancer and therapeutic opportunities}

Cancers, as a collection of diseases, are becoming more prevalent in modern society. One in four deaths in the USA are cancer related; however, the survival rate of patients with various cancers is improving, as indicated by a consistent decrease in the incidence of deaths per year caused by cancers in recent decades (Siegel, Naishadham et al. 2012). This is likely the result of improved awareness surrounding these diseases leading to earlier medical intervention rather than drastic improvements in treatment. Incremental advances in the way our medical systems approach these diseases have contributed to better therapeutic outcomes; however, relevant screening programmes are not available to everyone, and many cancers are only detected in the advanced stages of disease progression (World Health Organisation, WHO, 2014). The incidence of disease frequency is comparable worldwide, positioning cancers as a leading cause of death globally (Jemal, Bray et al. 2011) alongside cardiovascular complications, particularly in westernised countries (WHO). The New Zealand population follows similar trends, with 8,891 cancer related deaths from a total of 20,050 registered diagnoses in 2011 (NZ Ministry of Health, 2014). Cancers are characterised as diseases in which a subset of cells exist in a state of hyper-proliferative, unregulated growth as the result of accumulated mutations. These mutations may occur in a wide array of genetic elements; however, mutations are most commonly associated with sequence encoding proteins involved in cellular proliferation, differentiation and mitogenesis, mechanisms of DNA repair or the regulation of cell death (Hanahan and Weinberg 2000, Futreal, Coin et al. 2004, Stratton, Campbell et al. 2009).

Both the severity and prevalence of these diseases in society is a major driver for drug discovery and development in the pursuit of continually improved therapeutic outcomes. Traditionally, the treatment of many solid tumours has been limited to surgical resection. This approach, however, has deficiencies in its ability to remove all affected tissue, often 
leading to an eventual recurrence in tumour growth. Surgical methods are limited to solidtype tumours, with an additional difficulty in tissue removal from delicate organs such as in neoplasms of the brain, where critical functions may be severely impaired following resection (Black and Ronner 1987, Asthagiri, Pouratian et al. 2007). In the clinic, options are often limited to radiotherapy and chemotherapeutic approaches as our best treatments for cancers following the partial resection of any accessible tissue, especially in inoperable tissues or those with contiguous secondary lesions (DeAngelis 2001, Stupp, Mason et al. 2005). Cancers developing metastatic properties or those originating in the progenitor cells of circulatory systems, for example leukemic disease forms, escape surgical methods entirely due to delocalisation of cells with altered surface adhesion or chemokine response properties (Müller, Homey et al. 2001, Hood and Cheresh 2002, Kaplan, Riba et al. 2005, Bos, Zhang et al. 2009).

Most chemotherapeutic agents in clinical application for the treatment of cancers act on mechanisms involved in DNA replication or by targeting specific processes within cell division. Recent advances in genomics look promising for matching specific cancer genotypes to the most appropriate therapeutic agents (Al-Lazikani, Banerji et al. 2012); however, the need to continually improve on existing compounds or discover novel mechanistic targets remains prevalent for the development of effective future treatments. Chemotherapeutic approaches are cytotoxic in nature, and exhibit significant off-target effects as seen in yeast chemical-genetic screens (Kaelin 2005). This somewhat limits their efficacy in a therapeutic context. The majority of chemotherapeutic agents lack selectivity against cancerous cells and act instead as blunt, broad-spectrum cytotoxins against all rapidly dividing cells. This includes many healthy cell types within systemic tissue, leading to the aggressive side effects seen in patients undergoing chemotherapeutic regimes. Various proteomic and chemical-genetic approaches seek to understand these off-target 
effects in the hope of developing new, highly specific compounds with more tolerable side effect profiles (Evans and McLeod 2003).

\subsubsection{Microtubules as targets in chemotherapeutic approaches to cancer treatment}

Subcellular organisation within eukaryotic organisms is highly regulated by a number of structural proteins, which together make up the cytoskeleton. These proteins function as a unified system to maintain processes within the cell including cell division, intracellular transport, cell macrostructure and motility. Cytoskeletal elements contribute a major role in cell division by both meiosis and mitosis, with roles in organelle segregation, chromosomal separation and cytokinesis (Hayden, Bowser et al. 1990, Field, Li et al. 1999, Nogales 2000, Wittmann, Hyman et al. 2001). Microtubules are heterodimeric macropolymers consisting of relatively small $(100 \mathrm{kDa}) \alpha / \beta$-tubulin dimers (Nogales, Whittaker et al. 1999, Kavallaris 2010). These structures are key components of the cytoskeletal system, making them attractive targets for the disruption of hyper-proliferative cells in the treatment of cancers (Jordan and Wilson 2004). Both the $\alpha$ - and $\beta$ - tubulin monomers consist of three functional peptide domains - an N-terminus nucleotide-binding motif, a Cterminus region consisting of helical structural elements, and a smaller central domain of looped peptide structure involved in the formation of the microtubule lumen (Nogales, Whittaker et al. 1999). Each of these domains have specialized roles within the microtubule polymer. The central domain is comprised of primarily $\beta$-sheet secondary structure and contributes to favourable $\alpha$ - to $\beta$ - subunit associations (Nogales 2000). Within the Nterminal peptide region, a critical GTP binding motif acts as a catalytic site for regulating polymerisation behaviours (Nogales 2000). The C-terminal structural region contributes peptide structures involved in favourable binding of microtubule-associated proteins (MAPs) (Miyamoto, Perlman et al. 2003, Amos and Schlieper 2005). The formation of $\alpha / \beta$-tubulin heterodimers is a highly controlled process, mediated through interactions with 
multiple chaperone proteins and associated co-factors within the cytosol (Lewis, Tian et al. 1996, Tian, Huang et al. 1996, Liang and MacRae 1997).Tubulin heterodimers are formed by a polar interaction of each subunit in a head to tail addition, leading to the development of an extended linear structure known as the protofilament. Multiple protofilaments interact through a lateral association of individual heterodimer units within the filament, with a structural element known as the M-loop assisting in stabilising the major interdimer associations (Nogales, Whittaker et al. 1999). Cryo-electron microscopy (CEM) studies in both cell extracts and purified tubulin from Xenopus eggs has revealed polymorphisms in the number of protofilaments making up complete microtubule polymers; however, under physiological conditions the large majority of polymers consist of 13 associated protofilaments (Chrétien, Metoz et al. 1992). Microtubule assembly in vitro is altered in the presence of microtubule-targeting agents (MTAs), leading to polymers with varied protofilament stoichiometry (Andreu, Bordas et al. 1992, Matesanz, Rodriguez-Salarichs et al. 2011). The complete microtubule polymer is approximately $24 \mathrm{~nm}$ in diameter and surrounds a hollow, luminal space (Nogales, Whittaker et al. 1999). 
Nomenclature for describing tubulin macrostructure has been developed over time, based around conserved secondary structural features within folded tubulin-family peptide sequence. Residues 1- 206 are defined as the N-terminal region, residues 207- 384 the central domain followed by $385-415$ as the C-terminal structural region. Each major alpha-helix or beta-sheet structure is defined numerically in sequence from the aminoterminal of the peptide sequence as in figure 3 of Lowe, Li et al. (2001). This thesis utilises this nomenclature for any further reference to specific amino acid residues as below in figure 1.1.1.

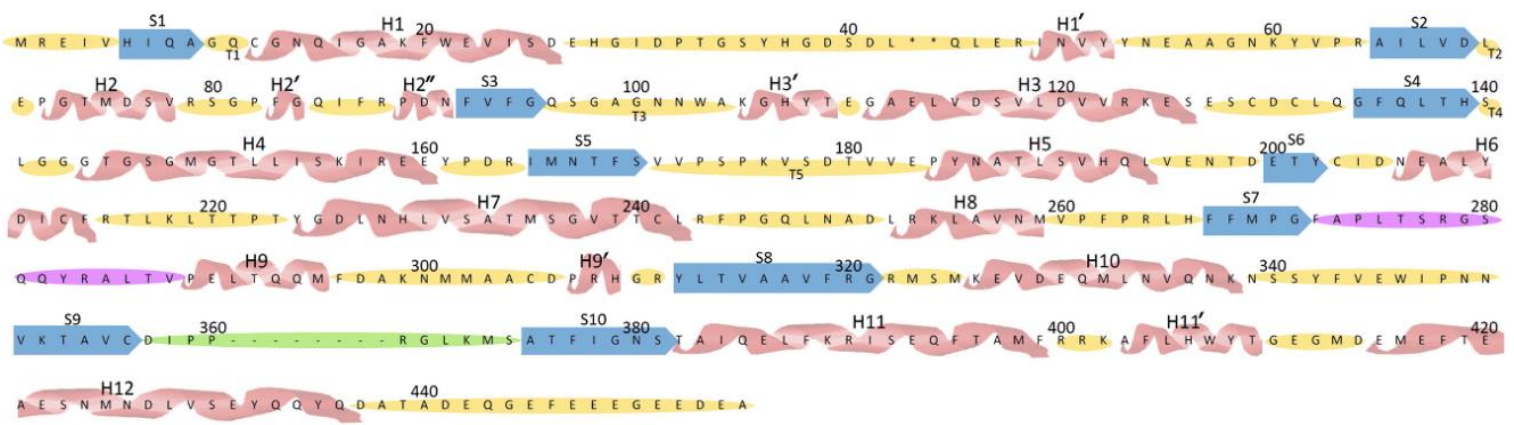

Figure 1.1.1 Linearized peptide sequence of $\beta$-tubulin. Sequence colour denotes structural elements. Sequence in pink indicates $\alpha$-helices, blue for $\beta$-sheets and looped sequence is represented in yellow. The M-loop, a region critical to interdimer stabilisations is shown in purple. Residues 1-206 constitute the N-terminal domain, the central domain indicated by residues 207-384 followed by the $\mathrm{C}$-terminal domain. Sequence elements are denoted according their corresponding structural role - $\mathrm{S}$ = Sheet, $\mathrm{H}=$ Helices. Nomenclature is assigned as published in (Lowe, Li et al. 2001) PDB JFF1. Figure taken with author's permission from Field, Díaz et al. (2013). 


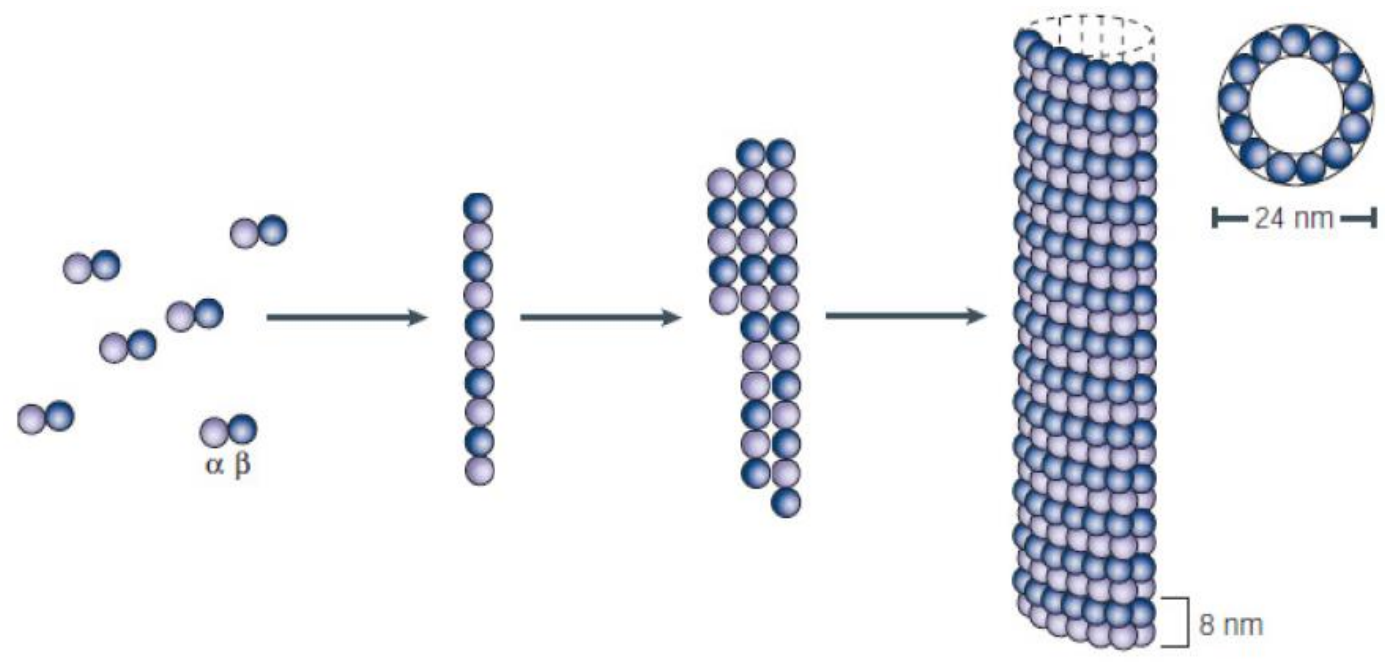

$\begin{array}{llll}\text { A } & \text { B } & \text { C } & \text { D }\end{array}$

Figure 1.1.2 Microtubule polymer-assembly. Individual tubulin monomers associate to form tubulin heterodimers (A). Heterodimers associate in a head to tail arrangement, forming linearized protofilament structures (B). Lateral associations between monomers within individual protofilaments $(\mathbf{C})$ leads to the generation of complete microtubule polymers $(\mathbf{D})$. These generally consist of thirteen protofilaments in vivo. Figure adapted from Jordan and Wilson (2004) with publisher's permission.

Microtubules were first identified in the early 1960's during the development of electron microscopy (EM) (Ledbetter and Porter 1963, Slautterback 1963). Soon after, microtubule subunits were isolated as the primary binding target of colchicine (Borisy and Taylor 1967, Weisenberg, Borisy et al. 1968), a compound with anti-mitotic properties now thoroughly characterised as a potent MTA. Through these early discoveries, chemical entities with binding affinity to microtubule proteins were quickly realised as valuable therapeutic agents for their powerful effects on disrupting normal cellular processes. Tubulin family proteins are among the most abundant protein species within all eukaryotic cells, with isoform distributions highly dependent on tissue specific regulatory processes (Sullivan 1988). Both the abundance of these proteins, their critical biological role and the newfound ability to perturb their function with specific compounds lead to the rapid development of first generation chemotherapeutic agents for the treatment of cancers. 


\subsubsection{Introduction to human tubulin genes}

Tubulin proteins are the products of a highly conserved gene family with ubiquitous presence across all eukaryotic genomes. On an evolutionary timescale, the critical nature of these proteins in the eukaryotic cytoskeleton has led to the development of multiple unique tubulin gene variants, each encoding tubulin proteins with highly specialised functional roles that differ between organisms, tissue types and even temporally within a single cell (Dumontet, Durán et al. 1996, Erickson 2007, Wickstead and Gull 2011). The gene family consists of $\alpha-, \beta-, \gamma-, \delta-, \varepsilon-, \zeta$ - and $\eta$-tublins (Dutcher 2001), alongside structurally similar prokaryotic gene variants encoding FtsZ - A tubulin-related GTPase (Erickson 1995, Du Toit 2014). In humans, the $\alpha$ - and $\beta$ - tubulin protein species make up the majority of cellular tubulin content necessary for cytoskeletal microtubules. Within these species, tissue-specific isotype expression results in a range of tubulins with varied biochemical properties dependent on unique peptide sequence, post-translational modifications or global protein stability (Ludueña 1993, Verdier-Pinard, Pasquier et al. 2009). Among the $\beta$-tubulins, $\beta \mathrm{I}$-tubulin is most widely distributed among various tissues, and many studies have shown MTA resistant cells develop altered isotype expression profiles, particularly in upregulation of $\beta$ III-tubulin (Kavallaris 2010). This phenomenon is seen similarly in cells resistant to Pel A and Lau (Kanakkanthara, Wilmes et al. 2011). As the primary $\beta$-tubulin isotype of the cell lines used in this study, the human $\beta \mathrm{I}$-tubulin gene was selected for the introduction of the D297I point mutation.

\subsubsection{Microtubule-Dynamics}

Microtubule-targeting agents have a history of relative success as cancer chemotherapeutics (Jordan and Wilson 2004), predominantly attributed to their potent effect on disrupting critical reorganisations of nuclear material, preventing cell cycle 
progression into the mitotic phase. Microtubules are highly dynamic in nature, demonstrating rapid rearrangements of tubulin heterodimers during periods of polymer extension or shortening. This behaviour, termed dynamic instability, describes fluctuations in heterodimer movement within the polymer macrostructure in response to changes in cellular requirements (Mitchison and Kirschner 1984, Nogales 2000).

The GTP binding motif of the N-terminal region in $\beta$-tubulin, termed the E-site, acts as a catalytic exchange site for modulating microtubule polymerisation behaviours. The exposed $\beta$-tubulin end, or plus end (+), of the microtubule is where a large portion of dynamic activity takes place (Akhmanova and Steinmetz 2008). The E-site of tubulin modulates the strength of lateral protofilament associations by a GTP/GDP induced allosteric shift between either a straight or curved conformation within the tubulin subunit (Nogales, Wang et al. 2003). When bound to the E-site, GTP induces a straightened conformation in tubulin, promoting a more favourable lateral interaction with tubulins in adjacent protofilaments. Bound GTP is then rapidly hydrolysed to GDP, introducing an energetic strain as $\beta$-tubulin shifts to re-adopt the curved conformation (Nogales, Wang et al. 2003). The rapid addition of a further GTP-bound tubulin heterodimer somewhat stabilises this strain, and allows lateral associations within the microtubule lattice to be maintained. The presence of GTP in the E-site of $\beta$-tubulins shifts dynamic equilibrium in favour of microtubule polymerisation, leading to overall elongations in polymer macrostructures. GTP associated with the (+) end of the microtubule serves to prevent depolymerisation of the microtubule and is consequently referred to as the GTP cap (Akhmanova and Steinmetz 2008). 


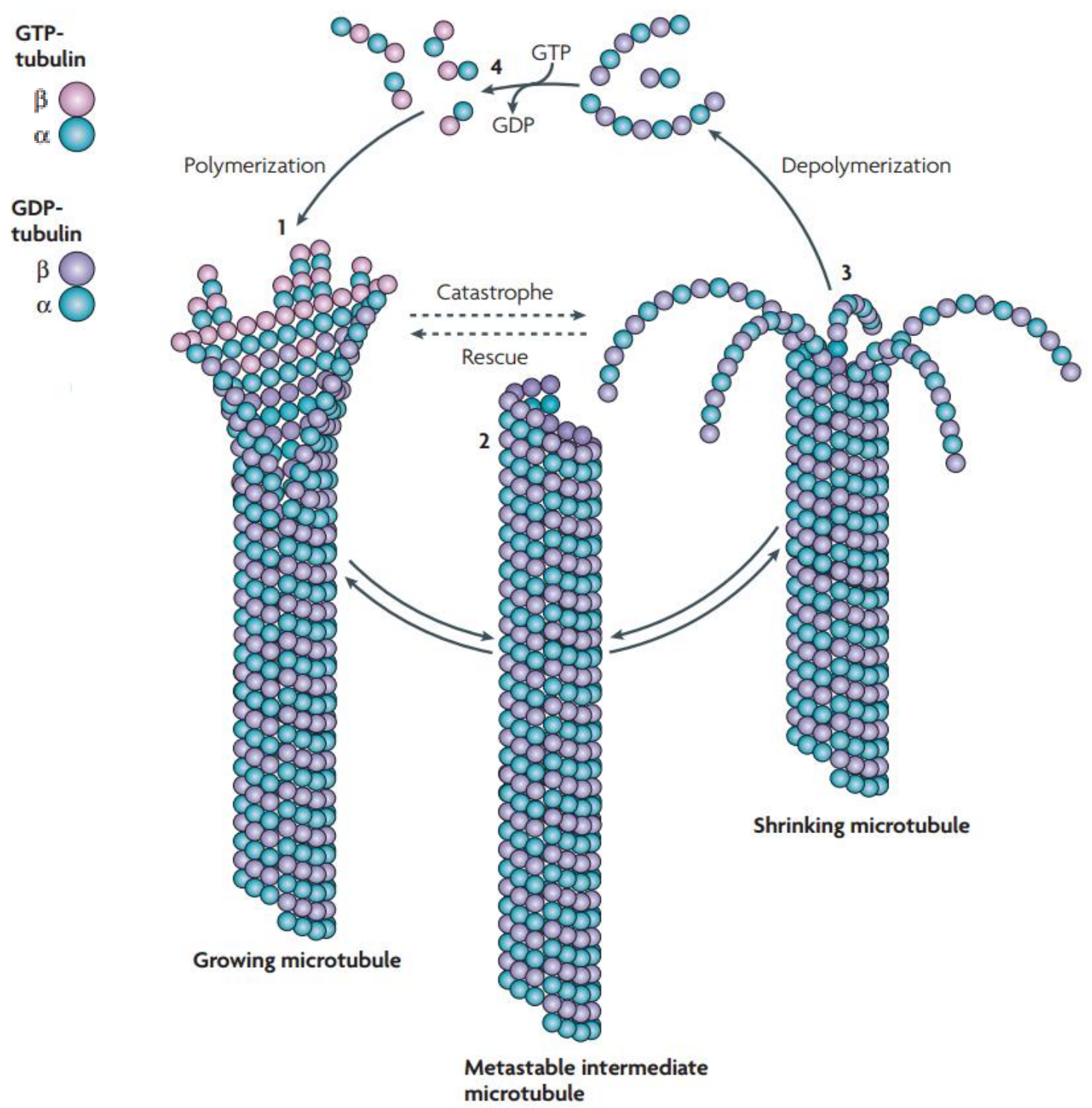

Figure 1.1.3 Microtubule dynamics and macropolymer structure. $\alpha$ - and $\beta$-tubulin monomers associate to form heterodimers. These then bind to GTP co-factors through the nucleotide binding motif in the N-terminal region of $\beta$-tubulin (pink). Polymerisation is favoured when GTP bound heterodimers are available in the cytosol, with rapid heterodimer addition to the microtubule polymer (1). GTP hydrolysis occurs soon after heterodimer addition, favouring depolymerisation as GDP-associated heterodimers (purple) return to a curved conformation. The addition of further GTP-associated heterodimers prevents this depolymerisation, thus a stabilised (2) or growing microtubule exhibits a characteristic GTP 'cap'. In the absence of GTP-associated heterodimers, the curved conformation of tubulin results in protofilaments appearing to 'peel' away from the lattice, resulting in microtubule depolymerisation (3). A shift in dynamics favouring polymerisation is known as 'rescue' while microtubule 'catastrophe' describes a shift towards depolymerisation of microtubule polymers. Figure adapted from (Akhmanova and Steinmetz 2008) with publisher's permission. 
In contrast, the lasting presence of GDP in the E-site of $\beta$-tubulin or the loss of the GTP cap favours disassembly of tubulin heterodimers. The curved conformation of GDPassociated-tubulin has low stability within the microtubule lattice in the absence of a GTP cap. Energetic strain within the lattice is relaxed as protofilaments peel away, leading to an overall reduction in total polymer length (Caplow, Ruhlen et al. 1994). This may occur as the result of reduced cytosolic GTP availability or when rates of GTP hydrolysis exceed GTP binding to the E-site (Janosi, Chretien et al. 2002). The N-terminal nucleotide binding motif of $\alpha$-tubulin, termed the $\mathrm{N}$-site, is non-exchangeable and may only be occupied by GTP. This results in lesser dynamic activity at the $\alpha$-tubulin subunits of heterodimers that are exposed in the minus (-) end of the microtubule. The presence of GTP exclusively in the $\mathrm{N}$-site promotes higher stability in the lateral associations with adjacent $\alpha$-tubulins (Nogales, Whittaker et al. 1999, Akhmanova and Steinmetz 2008). A transition from the growth state to one of depolymerisation is termed microtubule 'catastrophe' while reinstatement of lowered GTP hydrolysis rates and the associated shift towards microtubule polymerisation is known as microtubule 'rescue'(Jordan and Wilson 2004). Together, these processes allow global regulation of microtubule growth states as required for the maintenance or initiation of critical cellular processes.

Microtubules exhibit a second major behaviour whereby dynamic equilibrium is homeostatic and polymers exhibit no significant change in overall length. This is known as microtubule treadmilling, a flux process delicately regulated by GTP hydrolysis and tubulin isotype-specific binding stabilities that results in an equal rate of heterodimer gain from the (+) end of the microtubule as they are lost at the (-) end (Nogales 2000). Upon transition from the interphase to mitotic phase of cell division in somatic cells, the degree of dynamicinstability seen in microtubules increases significantly as polymers rearrange to form structural elements such as the mitotic spindle (Rusan, Fagerstrom et al. 2001). 


\subsubsection{Microtubule targeting agents}

MTAs interfere with microtubules dynamics through a number of mechanisms, preventing critical rearrangements in cytoskeletal elements and severely hindering many biological processes within the cell (Jordan and Wilson 2004). The cytotoxic effect of MTAs has been largely attributed to their effects on cell cycle progression. By disrupting the dynamic behaviour of microtubules, cells undergo mitotic arrest, leading to an induced cell death response through the intrinsic pathways of apoptosis (Jordan, Wendell et al. 1996). This is most readily seen in cells attempting to rearrange microtubules in order to form elements of the mitotic spindle and asters during cell division; actions that are severely inhibited in the presence of MTAs (Kelling, Sullivan et al. 2003). The ability to hinder these processes in a rapidly dividing cell population, such as in cancer, make MTAs ideal candidate molecules for use in the clinic. The relative success of many MTAs is attributed to this ability to induce mitotic arrest; however, mitosis-specific inhibitors such as those targeting specific mitotic kinases such as the Polo and Aurora kinases (reviewed in Taylor and Peters (2008)) have seen little clinical success (Komlodi-Pasztor, Sackett et al. 2012). This suggests that MTAs may in fact exert many of their potent in vivo cytotoxic effects by inhibiting microtubule dynamics during the interphase of the cell cycle, hindering processes of normal intracellular trafficking (Komlodi-Pasztor, Sackett et al. 2011, Komlodi-Pasztor, Sackett et al. 2012, Field, Kanakkanthara et al. 2014). New targeted therapeutics are beginning to emerge utilising antibody-MTA conjugates to introduce microtubule disruption within specific cell populations (Panowski, Bhakta et al. 2014) by targeting novel biomarkers of even the most difficult cancers (Pointer, Clark et al. 2014). These new generation therapeutics are beginning to enter the clinic (Younes, Yasothan et al. 2012), Ballantyne and Dhillon (2013), (Dhillon 2014) and show positive results. MTA compounds, however, remain essential for the mechanism through which these antibody-targeting therapeutics exert their toxicity. 
The vast majority of compounds characterised as MTAs have origins in natural products, with many then altered through synthetic chemistry for enhanced therapeutic properties. MTAs inhibit microtubule dynamics through two broadly defined mechanisms, leading to a categorisation as either a microtubule-destabilising agent (MDA) or microtubulestabilising agent (MSA). At low concentrations in vitro, both classes of compound appear to disrupt normal dynamic behaviour in microtubules, leading to mitotic arrest and apoptosis. At higher concentrations, each class of compound begins to produce characteristic behaviours in the dynamic instability of tubulin heterodimers. MDAs begin to reduce polymer length significantly, and may eventually deplete microtubule structures entirely (Jordan and Wilson 2004). MSAs in contrast, are able to stabilise heterodimer polymerisation at high concentrations. These agents induce polymerisation of heterodimers in the absence of GTP, or even those with exclusively N-site associated GDP. This leads to an in increase in polymer mass, the formation of microtubule bundles and a characteristic formation of multiple asters or aberrant centrosomes. The action of MTAs is determined by how each compound interacts within the tertiary structure of the $\alpha / \beta$-tubulin polymer. MTAs have a unique binding interaction with one of four distinct regions on $\beta$-tubulin (Huzil, Chik et al. 2008, Dumontet and Jordan 2010). Changes in microtubule dynamics as a result of drug treatment are dependent on the particular binding-region with which a compound associates. 


\subsubsection{Microtubule destabilising agents}

Many compounds demonstrate an ability to bind microtubules, inhibit polymerisation dynamics or even reverse assembly altogether. These MDAs associate with one of two regions on $\beta$-tubulin with names based on the first described compounds showing specific binding affinity to these regions. Colchicine-like compounds and vinca domain-binding agents describe two classes of compound with respective binding affinities either for the colchicine or vinca domains of $\beta$-tubulin. Each of these binding regions is located on the exterior region of the microtubule polymer wall. 


\section{Colchicine domain binding agents}

Colchicine-like compounds bind soluble heterodimers and effectively 'lock' the tubulin into a curved conformation. This decreases the stability of heterodimer integration into the microtubule lattice (Ravelli, Gigant et al. 2004), leading to depolymerisation, especially at higher concentrations. Despite the early identification of colchicine as a MDA as described in section 1.1.3, colchicine-like agents have yet to progress through clinical trials (Dumontet and Jordan 2010, Massarotti, Coluccia et al. 2012). Structural analogues that bind the colchicine site are, however, showing promise for treating cancers with selective vascular disruptions and are moving through late stage trials (Dumontet and Jordan 2010, Larocque, Ovadje et al. 2014). 

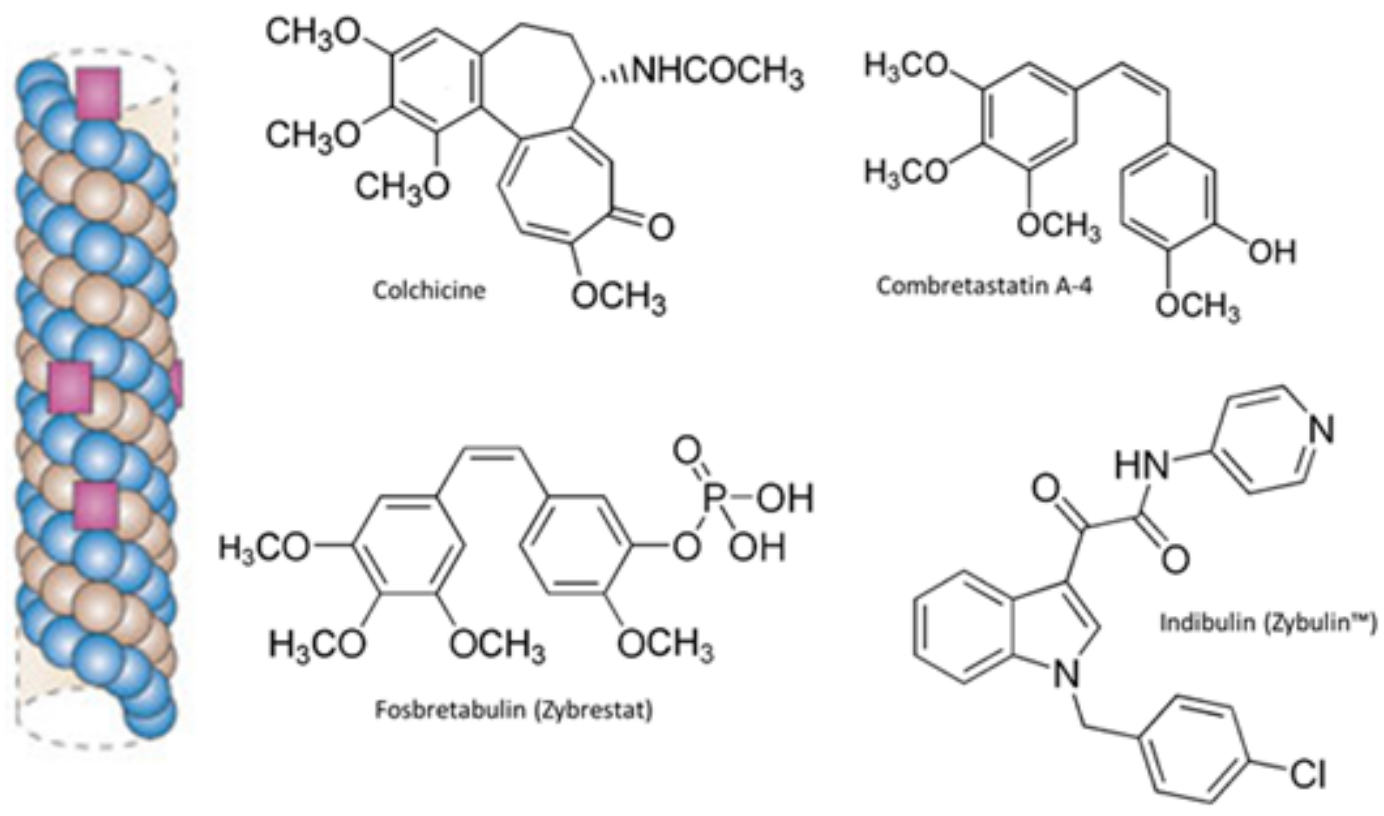<smiles></smiles><smiles>CC[C@H](N)C(=O)Nc1cc(/C=C\c2cc(OC)c(OC)c(OC)c2)ccc1OC</smiles>

Figure 1.1.4 The colchicine binding region. Colchicine associated compounds bind a region on the external microtubule wall, as indicated by the purple square. A selection of chemical structures of compounds known to associate with the colchicine binding domain of $\beta$-tubulin are also presented. Figure adapted from Dumontet and Jordan (2010) with publisher's permission. 


\section{Vinca domain binding agents}

Vinca domain binding agents such as vinblastine (approved 1961) and vincristine (approved 1963) have seen much success as MDAs in cancer therapies (Yue, Liu et al. 2010). Many structural analogues have since been developed and moved through for clinical use against a diverse range of cancers (Dumontet and Jordan 2010). Vinca domain binding agents associate directly with $\beta$-tubulin in the polymerised microtubule structure (Cormier, Knossow et al. 2010) and introduce disruptions in microtubule dynamics. It is thought that these compounds are able to interfere with regulation functions at the organisational centres of the (-) end of the microtubule, causing polymer fragmentation leading to inhibition of cell cycle progression (Yang, Ganguly et al. 2010). Despite their success, many vinca domain binding agents are susceptible to drug efflux pumps in cancer cells with MDR phenotypes; therefore, the development of analogues with improved properties is ongoing (Dumontet and Jordan 2010). 

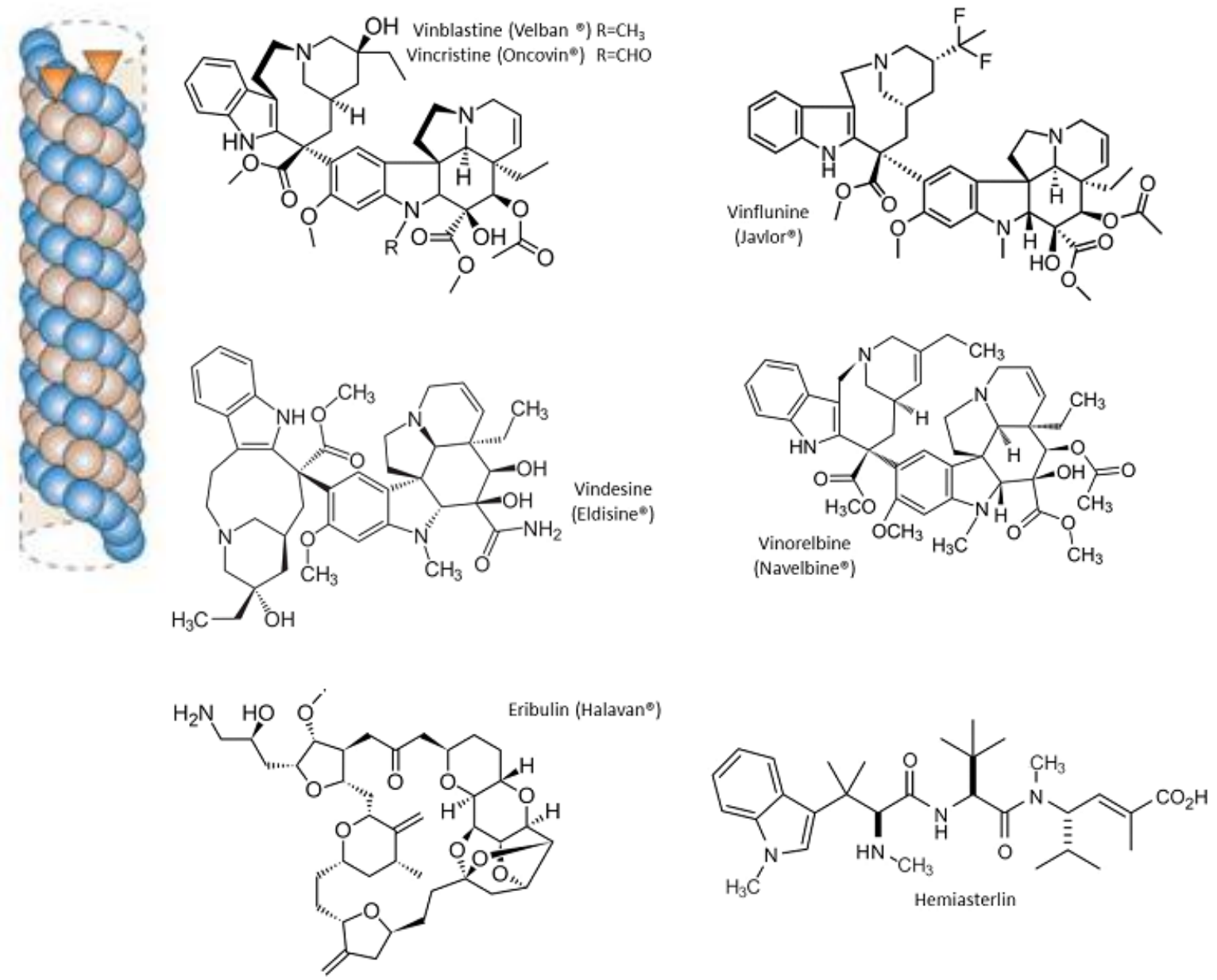

Figure 1.1.5 The vinca domain binding region. Vinca domain associated compounds bind a region on the external microtubule wall as indicated by the orange triangle. A selection of chemical structures of vinca domain binding compounds are also presented. Figure adapted from Dumontet and Jordan (2010) with publisher's permission. 


\subsubsection{Microtubule-stabilising agents}

The second major class of MTAs able to disrupt microtubule dynamics do so by stabilising interactions between tubulin heterodimers within the microtubule polymer (Dumontet and Jordan 2010). MSAs interfere with regular processes of dynamic instability by interacting with one of two distinct binding regions on $\beta$-tubulin. The majority compounds identified as MSAs interact with the well characterised taxane binding region, while two novel marine natural products, peloruside $\mathrm{A}(\mathrm{Pel} \mathrm{A})$ and laulimalide (LAU) display unique activities at a secondary site that has only recently been described (Huzil, Chik et al. 2008, Nguyen, Xu et al. 2010).

\section{Taxanes}

Taxane compounds represent the earliest known microtubule-stabilisers, with paclitaxel (PTX) first reported as demonstrating microtubule-stabilising properties in 1979 (Schiff, Fant et al. 1979), shortly after the compound was identified and shown to have potent antimitotic properties (Wani, Taylor et al. 1971). The compound was first isolated from the Pacific yew tree Taxus brevifolia in 1966. The potential anticancer activity of this compound was quickly realised, however its further development was limited by scarcity in the supply from raw biomass. Soon after, plant tissue culture methods were developed to produce an abundant precursor, 10-deacetyl-baccatin III, allowing semi-synthetic PTX to be produced for research and medical applications (Witherup, Look et al. 1990, Rohena and Mooberry 2014). PTX and its many derivatives stabilise microtubule polymers by binding to the taxane site of $\beta$-tubulin, located in the luminal space of the microtubule. These compounds associate with GDP-bound $\beta$-tubulin, relieving curvature in the protein's tertiary conformation, thus reinstating the favourable inter-protofilament interactions of the straightened tubulin form, and leading to increased polymer mass within a cell. (Elie-Caille, Severin et al. 2007). PTX has been in clinical application since 1992 as an antimitotic for 
treating a range of cancers, including those of breast, ovarian or lung tissues (Yue, Liu et al. 2010). PTX and its derivatives have undergone many iterations, improving on limiting pharmacological properties such as the compound's low aqueous solubility and need for a lipophilic delivery vehicle (such as Cremophor EL) (Gelderblom, Verweij et al. 2001) and susceptibility as a substrate for Pgp pump activity, especially in cells with MDR overexpression phenotypes (Gaitanos, Buey et al. 2004). There are a number of paclitaxel analogues in clinical application or late stage clinical trials, including those exploring advanced formulations or drug-nanoparticle-biomolecule conjugates (Rohena and Mooberry 2014). 

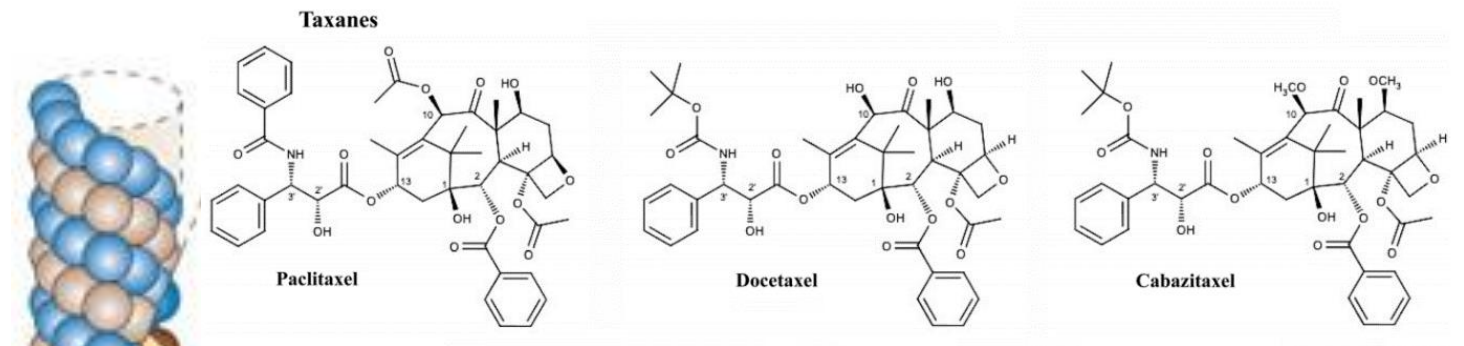

Taccalonolides

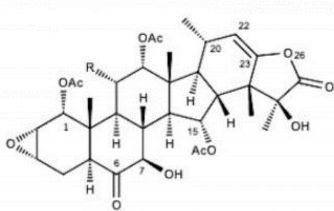

Taccalonolide $\mathbf{A} ; \mathbf{R}=\mathbf{O A C}$ Taccalonolide E; R= H

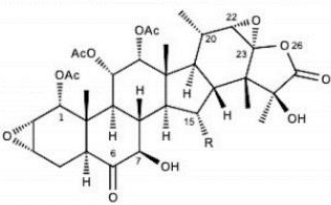

Taccalonolide AJ; $\mathbf{R}=\mathbf{O H}$ Taccalonolide AJ; $\mathrm{R}=\mathrm{OH}$
Taccalonolide AF; $\mathrm{R}=\mathrm{OAc}$

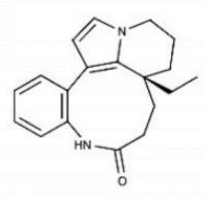

Rhazinilam

\section{Microbial Derived}

Epothilones

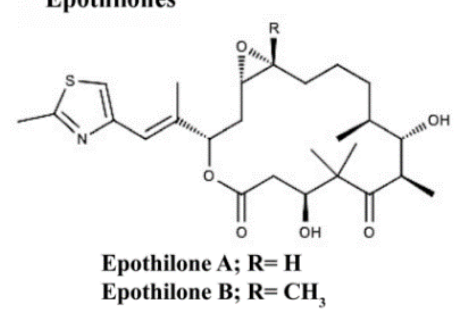

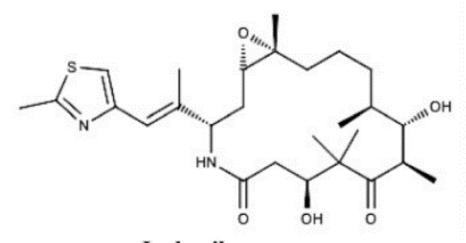

Ixabepilone

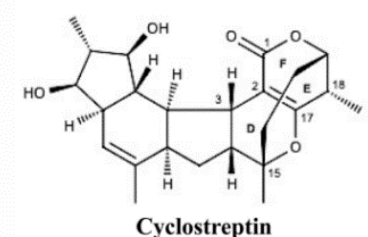

\section{Marine Derived}<smiles>C=C/C=C\C(C)[C@H](OC(N)=O)[C@@H](C)[C@@H](O)[C@@H](C)C/C(C)=C\C(C)[C@@H](O)C(C)/C=C\[C@H](O)CC1OC(=O)C(C)[C@H](O)C1C</smiles>

Discodermolide<smiles>C=C/C=C\C(C)C(OC(=O)/C=C\C=C/C(C)CC(C)C)C(C)/C=C\C(C)CC(C)/C=C\C</smiles>

Dictyostatin

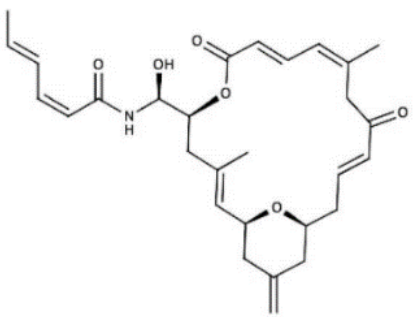

Zampanolide

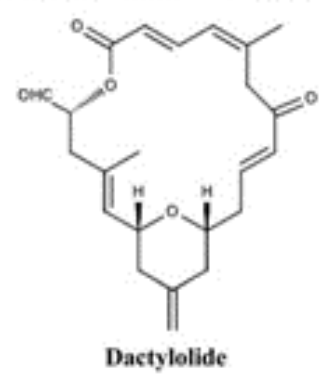

Figure 1.1.6 The taxane binding region. A selection of compounds with affinities to the taxane binding domain are also presented. These compounds associate to a region within the microtubule lumen as indicated by the yellow diamonds. Compounds have incredibly diverse chemical structure and come from plant, microbial and marine derived sources. Figure adapted from Dumontet and Jordan (2010) with publishers permission. 


\section{Epothilones}

Epothilones are a class of chemical agents originally isolated from the microbial species Sporangium cellulosum (Gerth, Bedorf et al. 1996). Epothilones A and B, the two major isolated products, demonstrated potent microtubule-stabilisation activities. This made them the first novel group of MSAs discovered since PTX (Bollag, McQueney et al. 1995). These compounds were of significant clinical interest as their potency was comparable to PTX and its derivatives, yet activity was retained in Pgp-over expressing MDR resistant cell lines. Epothilone B (azaepothilone B) is in fact a substrate for Pgp efflux; however, it is not a substrate for the breast cancer resistance protein BCRP/ABCG2 (Shen, Lee et al. 2011). The mechanism by which these compounds induce microtubule-stabilisation is similar to PTX, with binding affinity in the taxoid region of $\beta$-tubulin (Prota, Bargsten et al. 2013). Epothilones are readily produced through bioreactor fermentation, or a relatively simple synthetic route given the 16-membered macrolide structure exhibits less complexity than other natural products (Rohena and Mooberry 2014). A derivative of epothilone B, azaepothilone $\mathrm{B}$, has been in clinical applications for the treatment of refractory metastatic breast cancers since 2007 under the trade name Ixempra® (Bristol-Myers Squibb).

\section{Taccalonolides}

Taccalonolides are a class of compounds only recently described as having MSA activity. Taccalonolides A and E were found to induce characteristic microtubule polymerisation in treated cells; however, direct interactions with tubulin were not detected in these early studies (Tinley, Randall-Hlubek et al. 2003, Rohena and Mooberry 2014).These compounds are poor substrates for Pgp efflux pumps and retain activity in cell lines with mutations in the taxane binding region of $\beta$-tubulin and cell lines with varied $\beta$-tubulin isotype compositions (Peng, Risinger et al. 2011, Risinger, Westbrook et al. 2011). Investigations into the taccalonolide analogues taccalonolide AF and synthetic 
taccalonolide AJ have shown the ability to induce polymerisation of purified tubulin in vitro; however, the mechanisms by which this occurs appear somewhat dissimilar to PTX (Li, Risinger et al. 2011, Risinger, Westbrook et al. 2011). More recently, covalent interactions with $\beta$-tubulin in the taxane binding region have been identified and the unusual properties of these compounds are beginning to be better understood (Li, Risinger et al. 2014). Understanding the mechanisms by which these compounds induce microtubule-stabilisation may be incredibly valuable to the development of therapeutic MSAs in the future.

\subsubsection{Microtubule-stabilising agents of marine origin}

Marine organisms have to date been the most abundant source of novel microtubulestabilising agents (Rohena and Mooberry 2014). Compounds including the discodermolides, dictyostatins, zampanolides, dactylolides, eleutherobins, and sarcodictyins have all demonstrated the ability to induce microtubule-stabilisation through various mechanisms by interacting with the taxane binding region on $\beta$-tubulin (Field, Díaz et al. , Rohena and Mooberry 2014) favouring a straightening in tubulin conformations or improving protein-protein interactions via stabilisation of the M-loop region in tubulin (Prota, Bargsten et al. 2013). Interestingly, cyclostreptin (Buey, Calvo et al. 2007), zampanolide and dactylolide (Field, Pera et al. 2012, Larsen, Wilson et al. 2013), undergo unique covalent binding within the taxane region of $\beta$-tubulin, a novel pharmacological property that may be of future clinical value. Clinical development of most of these compounds has been slow given the complex chemical-syntheses involved in producing the compounds or their difficulty of isolation from natural sources. Two additional marine natural products, peloruside A (Pel A) and laulimalide (Lau) are the focus compounds of this study, and are covered in more detail in the following sections. 


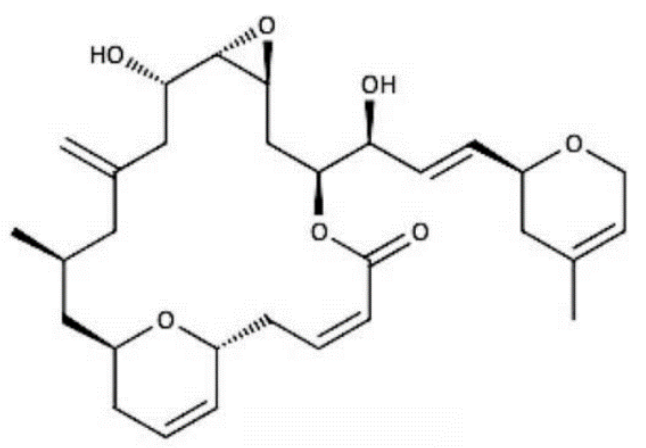

Laulimalide

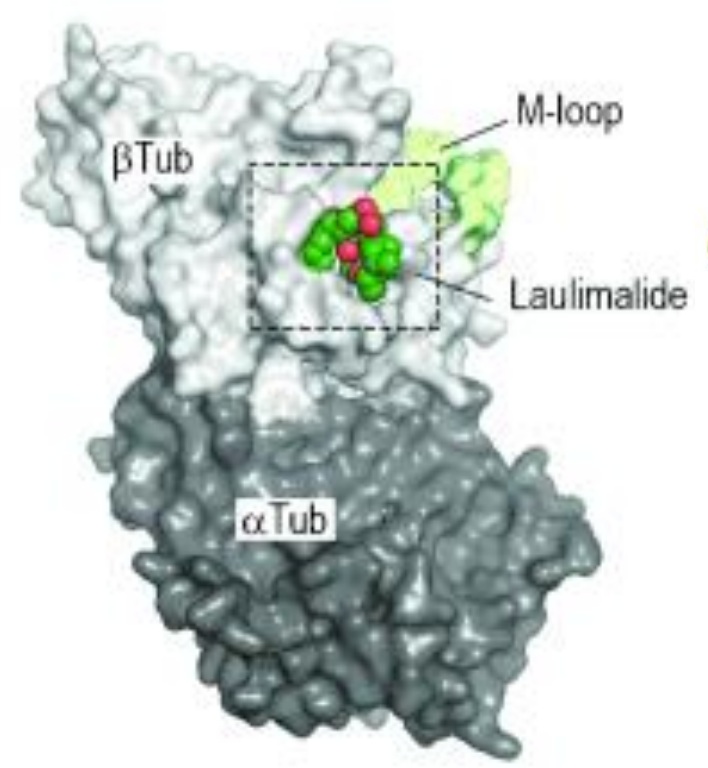

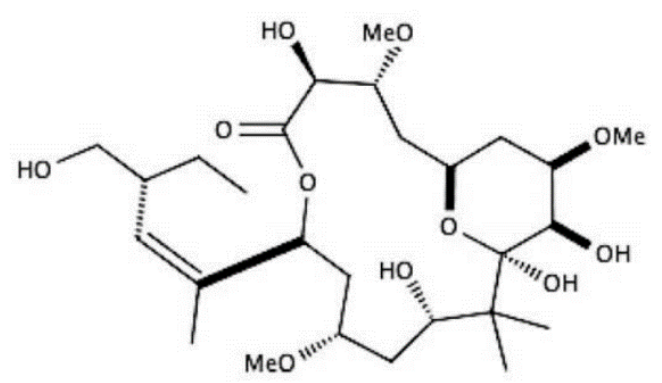

Peloruside A

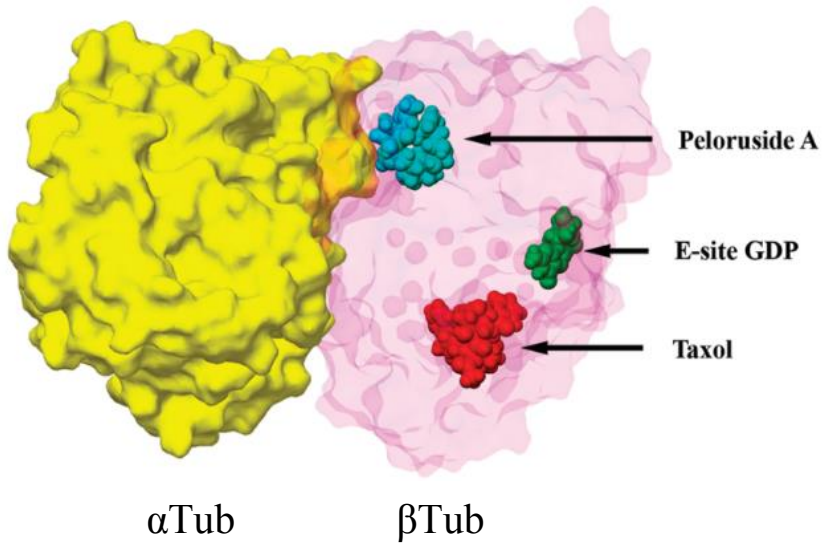

Figure 1.1.7 Chemical structures of two novel marine natural products LAU and Pel A. Both compounds are complex polyketides and demonstrate a potent ability to induce microtubule stabilisation in vitro. These compounds share a unique binding region within the $\beta$-tubulin monomer, localised in close proximity to the M-loop. 3D representations of the $\alpha / \beta$-tubulin heterodimer are presented, with both Lau (left) and Pel A (right) co-localised to the predicted binding region of $\beta$ tubulin. Pel A and Lau occupy a distinct region from that of PTX (right) Figures adapted from (Nguyen, Xu et al. 2010, Prota, Bargsten et al. 2014) with authors' permission.

Thus far, all natural products with MSA activity in clinical applications share a mechanistic region of action in the taxane site of $\beta$-tubulin. Two novel macrolides, Lau and Pel A act as potent microtubule-stabilising agents in vitro and produce similar disruptions in the dynamic-instability of tubulin as seen with traditional MSAs like PTX. These compounds, and their synthetic derivatives, have seen great interest in recent years following the initial characterisation of their novel binding region within $\beta$-tubulin. 
Lau, a 20-membered macrolide first isolated from the marine sponges Hyattella sp. and Cacospongia mycofijiensis in 1988 (Corley, Herb et al. 1988, Quinoa, Kakou et al. 1988). Initial characterisations found Lau to act as a potent MSA with nanomolar range bioactivity in vitro (Mooberry, Tien et al. 1999). Early binding studies revealed Lau did not inhibit the binding associations of radiolabeled or fluorescent paclitaxel derivatives (Pryor, O'Brate et al. 2002), suggesting an alternate mechanism was involved in Lau-induced microtubule polymerisation. In addition, Lau was found to retain activity in cell lines with mutations in the taxane binding region which conferred resistance to epothilone $\mathrm{A}$, epothilone $\mathrm{B}$ and PTX (Pryor, O'Brate et al. 2002) and those with Pgp overexpression and MDR phenotypes (Liu, Towle et al. 2007). Lau has been assessed in various tumour models; however, the compound has demonstrated mixed ability to inhibit tumour growth in vivo (Johnson, Tenney et al. 2007, Liu, Towle et al. 2007). This may be in part due to the relatively high lipophilicity of Lau (Mooberry, Tien et al. 1999), resulting in poor bioavailability of the compound in target tissues.

Pel A, a 16-membered macrolide first isolated from the New Zealand marine sponge Mycale sp. (West, Northcote et al. 2000) and was soon after characterised as a potent cytotoxin (Hood, Bäckström et al. 2001). The compound's mode of action for inducing cell death was identified through its characteristic MSA-like activity in vitro (Hood, West et al. 2002). Surprisingly, competition studies revealed Pel A shared a binding region with Lau, but not PTX (Gaitanos, Buey et al. 2004) and, like Lau, was a poor substrate for Pgp drug efflux mechanisms. This makes Pel A and its structural analogues of significant clinical interest as potential chemotherapeutics for use in cases of cancer with mutations in amino acids located within the taxane-binding region or those with MDR phenotypes against traditional MTAs. Studies into the synergistic capabilities of Pel A with taxane-region targeted compounds have indicated that combination therapy approaches utilising the novel 
binding region of compounds like Pel A or Lau along with the traditional taxoid site compounds may be a beneficial strategy in future chemotherapeutic regimes (Hamel, Day et al. 2006, Wilmes, Bargh et al. 2007, Kanakkanthara, Wilmes et al. 2011).

Both Pel A and Lau serve as excellent structural scaffolds for the development of nextgeneration antimitotic agents. These compounds act by a similar mechanism to the frontline chemotherapeutic agent PTX, yet exhibit novel pharmacological properties and maintain activity in drug resistant tissue types. In order to fully utilise these structures through adaptive synthetic-chemistry, complete characterisation of the novel binding region with which they engage is essential. Recently, a combination of hydrogen-deuterium exchange mass spectrometry (HDX-MS) (Huzil et al. 2008; Khrapunovich-Baine et al. 2011), mutational analysis of individual tubulin amino acid residues (Kanakkanthara et al. 2014), and x-ray crystallographic techniques (Prota, Bargsten et al. 2014) has begun to extend our knowledge of the critical drug-protein interactions through which both Lau and Pel A are able to stabilise tubulin polymerisation. By understanding these interactions, the potency and specificity of Pel A and Lau will be improved by alterations in chemical structure to improve on the clinical potential of these compounds. To progress these compounds toward clinical application, biological verification of the predicted drug-amino acid interactions within the Pel A/Lau binding region in these models is an essential insight useful for the rational development of synthetic Pel A/Lau analogues with improved therapeutic windows. 


\subsubsection{Research design}

The Pel A/Lau binding region of tubulin has received considerable interest in recent years given the potential this region represents for the development of novel chemotherapeutic agents. Traditionally, electron crystallography by CEM or x-ray diffraction techniques have been utilised to study microtubule structure or tubulin-ligand associations as in the case of solving the PTX-tubulin interaction (Lowe, Li et al. 2001). These approaches utilise a $\mathrm{Zn}^{2+}$ surface to stabilise the formation of 2-D tubulin crystal sheets in order to generate diffraction data. Unlike PTX, Lau does not produce a 2-D crystalline tubulin sheet on $\mathrm{Zn}^{2+}$ (Thepchatri, Cicero et al. 2005). This prevented elucidation of the structure by x-ray crystallography, thus early insights into the Pel A/Lau binding region on $\beta$-tubulin relied on alternate methodologies. Huzil, Chik et al. (2008) utilised HDX-MS peptide mapping techniques to avoid the problematic aspects of generating $\mathrm{Zn}^{2+}$ crystal sheets in initial investigations into the binding modality of Pel A, generating evidence in support of a novel region within $\beta$-tubulin that may be a useful target for the stabilisation of microtubules. These studies were followed by refined models of Pel A/Lau binding within the proposed region, generated by computational methods, mass shift perturbation and radiolabeled $\left[{ }^{3} \mathrm{H}\right]-$ Pel A affinity techniques (Bennett, Barakat et al. , Nguyen, Xu et al. 2010); however, each group offered an alternate prediction of Pel A orientation within the binding region. Further HDX-MS data generated by Khrapunovich-Baine, Menon et al. (2011) supported the initial binding region proposed by Huzil, Chik et al. (2008) as this region was highly protected from deuterium incorporation by both Pel A and Lau, and suggested these compounds strongly enhance interprotofilament association between $\beta$-tubulins through an induced stabilisation of the M-loop structural region (Khrapunovich-Baine, Menon et al. 2011). 
More recently, Prota, Bargsten et al. (2014) unified aspects of these proposed models through elucidation of the tubulin ternary crystal structure while in complex with each of Pel A, Lau and Epothilone A. This was achieved through the crystallisation of $\alpha / \beta$ tublin in complex with the stathmin-like protein (SLD) RB3, and tubulin tyrosine ligase (TTL) (Prota, Bargsten et al. 2013, Prota, Magiera et al. 2013). Crystals suitable for study by xray diffraction were produced through the use of this complex, thus avoiding obstacles associated with $\mathrm{Zn}^{2+}$ tubulin crystal sheets. These studies provided an in depth model for compound modality within the novel Pel A/Lau binding region of $\beta$-tubulin, and has allowed further predictions to be made regarding the functional role of individual amino acid-drug associations. A mechanism through which Pel A or Lau may induce microtubulestabilisation has been proposed alongside these predictions - A critical development in our understanding of this novel MSA-target site in $\beta$-tubulin. In addition, Churchill, Klobukowski et al. (2014) have recently modelled the binding association of $\beta$-tubulin with Lau in the presence of an adjacent protofilament, observing a large number of $\mathrm{H}_{2} \mathrm{O}$ mediated bonding interactions in the consensus region. With interactions from Lau bridging the interdimer space, residues E53, K122, E125, S126 and D128 on the adjacent $\beta$-tubulin were identified as contributors in the binding association. 

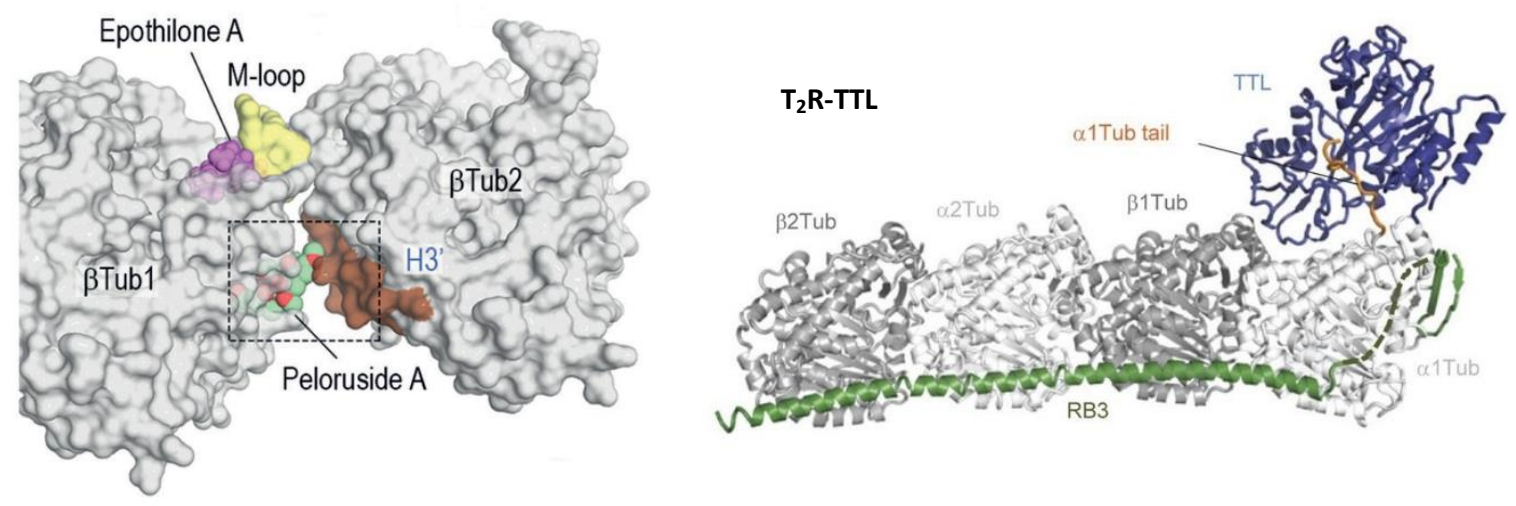

Figure 1.1.8 The binding region of Pel $\mathrm{A}$ and Lau. Peloruside $\mathrm{A}$ is modelled within the predicted Pel A/Lau binding region of $\beta$-tubulin (left) alongside the $\alpha / \beta$-tubulin-RB3-TTL complex $\left(\mathrm{T}_{2} \mathrm{R}\right.$ TTL) used to elucidate the $\beta$-tubulin crystal structure while associated with Pel $\mathrm{A} / \mathrm{Lau}$ ligands (right). Figure adapted from (Prota, Bargsten et al. 2014) and (Prota, Magiera et al. 2013) with publisher's permission.

The research presented within this thesis aims to explore the role of aspartic acid 297 (D297) located within the Pel A/Lau binding region. This amino acid has a predicted functional role in stabilising the binding of Pel A and Lau as first predicted by Huzil, Chik et al. (2008), Khrapunovich-Baine, Menon et al. (2011) and recently Prota, Bargsten et al. (2014). Recently Churchill, Klobukowski et al. (2014) have provided additional computational studies noting a contribution to ligand binding energy from this region. Initial computer modelling based on the $\mathrm{Zn}^{2+}$ tubulin structure suggested this particular amino acid may be involved in Pel A/Lau binding and was selected for this investigation before the Pel A/Lau$\beta$-tubulin crystal structure of Prota, Bargsten et al. (2014) was available. In the present study, we seek to provide biological evidence for the role of D297 in the Pel A and Lau induced the stabilisation of microtubules.

Studies in cell lines resistant to Pel A and Lau have identified distinct point mutations in $\beta I$-tubulin sequence that result in reduced sensitivities to both compounds. These include mutations in amino acids A298, R308, Y342 and N339 (Begaye, Trostel et al. 2011, Kanakkanthara, Eras et al. 2014). Consistent with the crystallographic predictions of Prota, 30 
Bargsten et al. (2014), these particular amino acids are identified as having functional roles in the binding association of both Pel A and Lau.

Kanakkanthara, Eras et al. (2014) describe the partial resensitisation of A298 or R308 mutant cells that are resistant to Pel A and Lau when a wild-type $\beta$ I-tubulin is introduced by means of transient transfection. As no biological evidence is available detailing the many predicted drug-amino acid interactions in $\beta$ I-tubulin made by Prota, Bargsten et al. (2014), the present study utilises a reverse approach to that used by Kanakkanthara, Eras et al. (2014), introducing point mutation-containing $\beta$ I-tubulin subunits to the tubulin population in a mammalian cell line, producing a model useful for the assessment of the biological role that individual amino acids play in these particular drug-protein binding associations. By introducing mutant D297I- $\beta$ I-tubulin, a subunit with an isoleucine substitution at position 297, transfected cultures were assessed for changes in sensitivity to the compounds Pel A, Lau, PTX and IXA. In addition, this research examines mutant protein expression in this $\beta$ I-tubulin point mutation model, assessing its suitability for use in characterising individual amino acids in the Pel A/Lau-tubulin binding association. 


\subsubsection{Aims of this research}

The aims of the research presented in this thesis are as follows:

\subsubsection{Examine the functional role of the D297 amino acid residue of human $\beta I$ - tubulin in the predicted binding association of Pel A and Lau}

Utilising a mammalian cell line as a model for MSA-induced polymerisation dynamics, the D297 amino acid residue of $\beta$ I-tubulin will be assessed for functional involvement in the $\beta I$-tubulin-Pel A or Lau binding association. By introducing a mutant $\beta$ I-tubulin isoform, the particular involvement of D297 in Pel A/Lau drug binding will be assessed through observing any change in the sensitivity of a cell line in response to Pel A or Lau. The predicted role of D297 in the stabilisation of Pel A/Lau binding by Prota, Bargsten et al. (2014) suggests a point mutation at this position may disrupt favourable hydrogen bonding interactions between Pel A/Lau and $\beta$ I-tubulin in the wild-type isoform. It is hypothesised that the introduction of the mutant D297I- $\beta$ I-tubulin through transfection may confer resistance to Pel A and Lau, allowing a level of dynamic-instability to be maintained within microtubule polymers in the presence of either compound. This research will provide evidence in support of or against an interaction between D297 and Pel A and Lau. The extent of introduced disruption may differ between each compound, providing further insight into the mechanism by which each of these compounds stabilise tubulin.

A thorough investigation into the concentration-response of wild-type, mock-transfected and D297I- $\beta$ I tubulin-transfected cells in the presence of Pel A and Lau may reveal altered responses in the proliferative characteristics of transfected cultures. This will allow any shift in the concentration-response phenotype of D297I- $\beta$ I tubulin-transfected cells to be considered as a direct result of the D297I mutation presence within tubulin when compared to the concentration-response of cells with WT $\beta$ I-tubulin only. This will indicate whether this particular amino acid substitution is likely involved in the binding association of Pel 
$\mathrm{A} / \mathrm{Lau}$ to human $\beta \mathrm{I}$-tubulin and provide direct biological support for or against a role of D297 as predicted by (Prota, Bargsten et al. 2014).

\subsubsection{Determine optimum transfection parameters for mutant $\beta I$-tubulin expression in a mammalian cell line}

In order to detect any changes in the sensitivity of cells transfected with a mutant $\beta$ I-tubulin, it is hypothesised that should the D297I point mutation confer a resistance phenotype, the magnitude of this resistance would be dependent on the relative abundance of mutant $\beta I-$ tubulin present within the whole cell population. A mammalian cell model expressing the mutant $\beta$ I-tubulin isoform in abundance may show a large shift in concentration-response should the point mutation play a functional role in the binding association of Pel A/Lau. This study will determine parameters for maximising mutant protein expression across the population for use in this model.

The presence of mutant $\beta$ I-tubulin within whole cell samples of transfected cells will be assessed by Western blotting to examine the abundance of mutant $\beta$ I-tubulins. This will allow for correlation of any concentration-response changes to the presence of mutant $\beta I-$ tubulins in transfected cells treated with Pel A or Lau.

\subsubsection{Examine the integration of exogenous mutant tubulin subunits into polymerised microtubules}

Tubulin-family proteins play critical roles in the maintenance of normal cellular processes. For this reason, it was essential that the introduced mutant protein isoform was biologically functional and that presence of this protein does not affect the viability of transfected cells. Live-cell confocal microscopy will be utilised to examine the presence of the mutant $\beta I-$ tubulin subunits in situ, within polymerised microtubules. Successful integration of the mutant protein isoform into the tertiary microtubule structure is critical for allowing assessment of the D297 amino acids role in response to Pel A or Lau. 


\subsubsection{Examine the concentration-response of D297I mutant transfected cells in response to drugs targeting the taxane region of $\beta$-tubulin}

By assessing cells transfected with the mutant $\beta$ I-tubulin against both IXA and PTX, we may determine whether the presence of the D297I point mutation in $\beta I$-tubulin has any effect on the global conformation of the protein. These drugs target a region of $\beta$ I-tubulin situated a significant distance away from the Pel A/Lau binding region, and thus it is hypothesised no change in the $\mathrm{IC}_{50}$ with these compounds should be observed. If this hypothesis is correct, these investigations will provide further support for any change in the $\mathrm{IC}_{50}$ of cells with D297I- $\beta \mathrm{I}-$ tubulin in response to Pel and Lau as a result of mutation presence. 


\section{Chapter 2. Exogenous human $\beta 1$-tubulins as tools for investigations of structural activity relationships.}

\section{$2.1 \quad$ Introduction}

The material presented within chapter two of this thesis relates to experimental design and the generation of an experimental model for the investigation of D297 in the binding association of pel A and Lau.

\subsubsection{Mammalian cell models for the study of drug-protein interactions}

Mammalian cells are a valuable tool for the study of drug-target interactions, especially for the investigation of singular protein components within a complete cellular environment. Cell lines are often criticised on aspects of their physiological relevance, particularly in the study of complete disease states. For the purposes of this investigation, cell lines remain incredibly useful for generating insights into the specific binding associations of Pel A/Lau and tubulin as we are solely focused on drug-protein binding interactions that do not require a complete biological system.

Human cells maintained in culture produce tubulin and microtubule polymers that exhibit normal processes of dynamic-instability. As described in chapter 1, microtubule polymerisation from purified tubulin heterodimers demonstrates slight alterations in quaternary structure from that of physiological tubulin. This makes tubulin in a cellular environment preferable for the study of these particular drug-protein interactions.

Introducing genetic manipulations to produce mutant tubulin variants is slightly more challenging in a mammalian system when compared to other more amenable organisms such as the budding yeast Saccharomyces cerevisiae. This is the key reason many investigations into the Pel A/Lau binding region thus far have been carried out in yeasts. While more challenging, mammalian cells are far more sensitive to MSAs in vitro than 
yeast, which require drug concentrations in the low micromolar range to induce significant G2/M phase arrest. This makes detection of subtle concentration-response changes in the presence of mutant $\beta$ I-tubulin more difficult, along with consuming far more drug of interest. Natural products such as Pel A and Lau are scarcely available for research purposes, thus the challenge of introducing genetic manipulations in mammalian cells is justified.

\subsubsection{Introduction to transfection and exogenous gene expression}

In order to introduce $\beta$ I-tubulin subunits containing the D297I point mutation into the cell model, this study made use of standard cationic lipid-based transfection methods. Episomal sequence encoding the mutant $\beta$ I-tubulin of interest expressed contiguously with a small green fluorescent protein (GFP) variant was utilised as a marker for successful transfection, and to monitor aspects of both mutant abundance and microtubule morphology. Plasmid DNA encoding sequences of interest may be introduced to cationic lipid-based transfection reagents to form transfection complexes that may move into cells by mechanisms of endocytosis. For a thorough review of transfection as an approach for introducing exogenous DNA to cells, see Chesnoy and Huang (2000). 


\subsubsection{Predicted interactions of Pel A/Lau with $\beta$ I-tubulin}
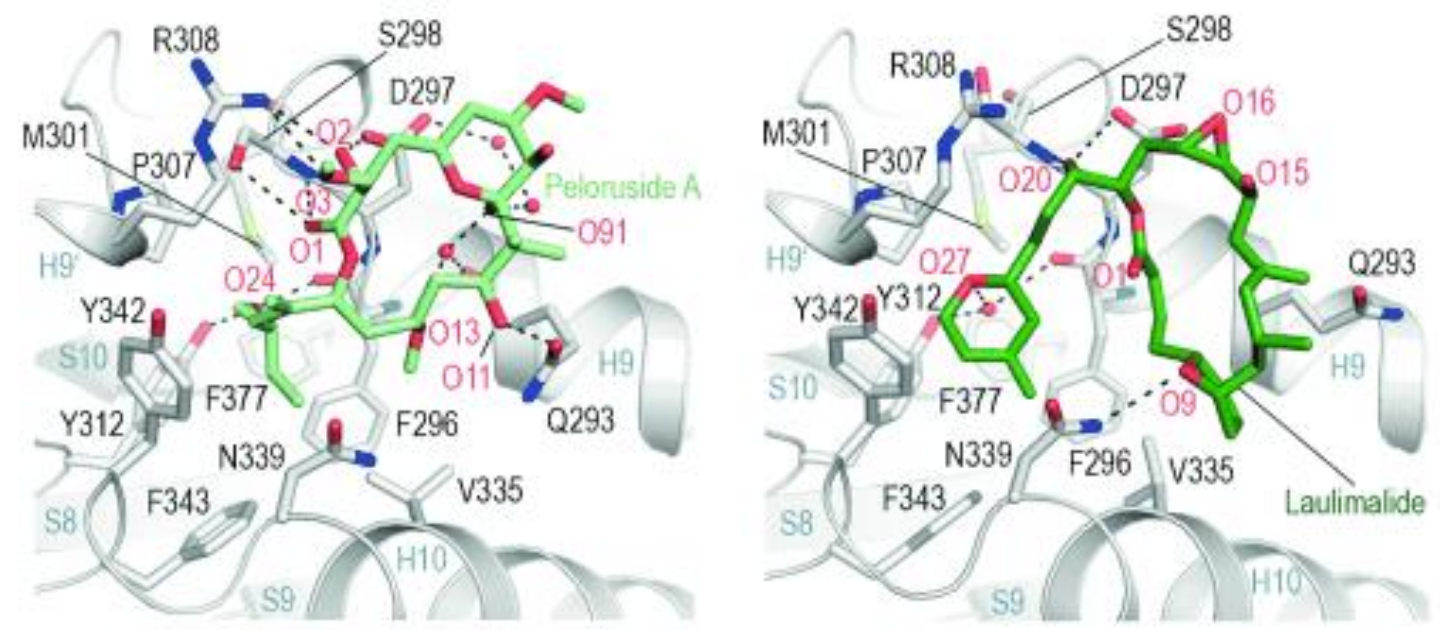

Figure 2.1.1 Binding interactions of Pel A and Lau. Peloruside A (left) and Laulimalide (Right) within the predicted Pel A/Lau binding region of $\beta$-tubulin. Models proposed by Prota, Bargsten et al. (2014) provide a detailed insight into the discrete interaction mechanisms through which both Pel A and Lau associate with $\beta$-tubulin. D297 is predicted to share hydrogen bond interactions between oxygen $2(\mathrm{O} 2)$ of peloruside (left), and oxygen $20(\mathrm{O} 20)$ of laulimalide (right). Figure adapted from (Prota, Bargsten et al. 2014) with author's permission.

Based on the crystallographic studies of Prota, Bargsten et al. (2014), both Pel A and Lau interact with $\beta$-tubulin in a binding pocket defined by amino acid residues Q293, F296, P307, R308, Y312, V335, N339, Y342 and F343. Within this region, Pel A is predicted to interact via hydrogen bond formations with amino acids S298, D297/R308, R308, Q293 and Y312 by oxygen atoms O1, O2, O3, O11 and O24 respectively. Similarly, Lau is proposed to associate via the formation of hydrogen bonds between amino acids N339, D297/S298 and oxygen atoms O9 and O20, along with an additional water-mediated hydrogen bond interaction between $\mathrm{O} 27$ and the free hydroxyl present in the side chain of Y312 (Prota, Bargsten et al. 2014).

The D297I point mutation was selected for investigation based on insights from computational analysis and docking studies initially carried out by Huzil, Chik et al. (2008) and Khrapunovich-Baine, Menon et al. (2011) generated through HDX-MS experimental 
data. Although selected for investigation before the elucidation of the crystallographic structures of $\beta$-tubulin in complex with Pel A/Lau ligands by Prota, Bargsten et al. (2014), these insights further implicated D297 as functional in ligand binding. Together these studies provide a basis for a need to characterise D297 interactions with Pel A/Lau through biological investigations, as carried out in the present study. 


\subsubsection{Aims of this chapter}

A) Optimise transfection parameters to maximise tGFP-D297I- $\beta$ I-tubulin and tGFP- $\beta$ I-tubulin expression throughout a mammalian cell population

To investigate the role of the D297 amino acid residue in the binding association of Pel $\mathrm{A} / \mathrm{Lau}$ in vitro, a model with ubiquitous point mutant $\beta \mathrm{I}$-tubulin distribution will most accurately reveal changes in concentration-response as a consequence of said point mutation presence. This chapter aims to determine transfection parameters for producing a model cell population that maximises the distribution of $\beta$ I-tubulin point mutation in the culture as consistently as possible, while avoiding artefacts in cell-sensitivity to drug treatment in cell proliferation assays.

\section{B) Quantify exogenous expression of mutant $\beta I$-tubulins in the transfected cell population of paired concentration-response experiments}

The second aim of this chapter is to determine the relative abundance of $\beta$ I-tubulin present in the cell population with the tGFP-D297I and tGFP-WT tubulin fusion sequences in order to assess the magnitude of changes seen in paired concentration-response experiments. Any phenotypic response may be dependent on mutant protein abundance within individual cells or alternatively, mutation presence within the population may allow for retention of dynamic microtubule behaviours in the presence of a drug.

\section{C) Examine the morphology of mutant tubulin-containing subunits within polymerised microtubules}

To support any correlations between mutant $\beta$ I-tubulin presence and phenotypic changes in concentration-response to drug, the introduced mutant $\beta$ I-tubulin must be functionally integrated within microtubules. The third aim of this chapter is to assess exogenous $\beta \mathrm{I}-$ tubulin by confocal microscopy to determine if point-mutation containing subunits are in fact integrated into microtubule structures rather than simply expressed as inactive protein 
in the cytosol. Microtubules containing mutant elements while involved in regular cellular functions suggest the presence of the mutation does not severely hinder these processes, lending support for the independent assessment of cell proliferation in concentrationresponse studies as a true indicator of changes in drug-protein interaction. 


\subsubsection{Materials and methods}

\subsubsection{Compounds}

Peloruside A (Pel A) and laulimalide (Lau) were kindly provided by Associate Professor Peter Northcote and Dr Jonathan Singh of the School of Chemical and Physical Sciences, Victoria University of Wellington, New Zealand. The marine sponges Mycale hentsheli and Cacospongia mycofijiensis were collected from the Pelorus Sound, Marlborough Sounds, New Zealand, and 'Eua and Vava'u, Tonga respectively. Mycale hentsheli was utilised as source material for purification of Pel A, and Cacospongia mycofijiensis served as raw extraction material for the purification of laulimalide. Once purified, these natural products were dissolved at $10 \mathrm{mM}$ in DMSO and stored at $-80^{\circ} \mathrm{C}$. Paclitaxel was purchased from Sigma Chemical Co. (St. Louis, MO, USA) under the trading licence Taxol®. Ixabepilone was purchased as Ixempra ${ }^{\circledR}$ from Bristol-Myers Squibb (USA). All compounds were stored as $10 \mathrm{mM}$ stock solutions and diluted to $10 \mu \mathrm{M}$ working stocks in RPMI-1640 medium (Invitrogen). All working stocks were stored at $-20^{\circ} \mathrm{C}$.

\subsubsection{Cell culture}

Two cell lines, the human embryonic kidney cell line (HEK293) and the 1A9 cell line, a sub-clone of the A2780 human ovarian carcinoma cell line, were utilised in this investigation, with each subject to regular PCR-based screening for mycoplasma contamination. Sterile culture and maintenance of each cell line was carried out in a Logic Labconco® Purifier® Biological Safety Cabinet (Total Lab Systems Ltd, New Zealand). HEK293 cells were cultured in DMEM medium (Invitrogen) supplemented with 10\% fetal calf serum (FCS) (Invitrogen), $2 \mu \mathrm{M}$ Glutamax ${ }^{\mathrm{TM}}$ (Life Technologies) and 100 units/mL penicillin/streptomycin (Invitrogen) antibiotic unless otherwise stated. 1A9 cells were cultured in RPMI-1640 medium (Invitrogen) supplemented with $10 \%$ fetal calf serum (FCS) 
(Invitrogen), .25 units/mL human recombinant insulin (Sigma Chemical Co. St. Louis, MO, USA) and 100 units/mL penicillin/streptomycin (Invitrogen) antibiotic supplementation. Cells were grown in $75 \mathrm{~cm}^{2}$ culture flasks (Falcon®, Invitro Technologies) in a humidified incubator (SANYO Electric Co., Ltd) at $37^{\circ} \mathrm{C}$ with a $5 \% \mathrm{CO}_{2}$ supplemented air atmosphere. Cells were harvested or passaged at approximately $75 \%$ to $80 \%$ confluence. Flasks were aspirated and the cells detached via the addition of $1 \mathrm{~mL}$ TrypLE ${ }^{\mathrm{TM}}$ Express (Life Technologies) followed by a 4 min incubation at $37^{\circ}$. Cells were then suspended in growth media and centrifuged for 5 minutes at $300 \mathrm{~g}$ to remove the TrypLETM containing supernatant before seeding into new culture vessels. Frozen cell stocks were maintained in liquid nitrogen in Cryo. s cryotubes (Greiner Bio-one) at $1 \times 10^{6}$ cells/ $\mathrm{mL}$ in culture medium with $10 \%$ DMSO as a cryoprotectant. All cell cultures were disposed of within 15 passages from the initial recovery from cryogenic storage to maintain genetic integrity over the duration of this investigation.

\subsubsection{Mammalian expression vector design and preparation}

The PrecisionShuttle mammalian vector with C-terminal TurboGFP flag pCMV6-AC-GFP (Origene Technologies, Rockville, MD, USA) was selected as the backbone expression vector for the expression of human tGFP- $\beta 1$-tubulin in mammalian cells driven by the highly active cytomegalovirus promoter (CMV). The single point mutation in amino acid residue 297 of the $\beta 1$-tubulin sequence in plasmid RG203629 (Origene) was introduced during cloning processes by Mutagenex (NJ, USA), whereby the selected codon for aspartic acid was substituted with isoleucine and the complete construct transformed into E. coli. Both mutant tGFP-D297I- $\beta 1$-tubulin and the parental tGFP- $\beta 1$-tubulin-transformed bacterial cultures were used to inoculate sterile Lysogeny broth (LB) agar (1\% Bactotryptone, $5 \%$ Bacto-yeast extract $1 \% \mathrm{NaCl}, 1 \%$ agar) with $100 \mu \mathrm{g} / \mathrm{mL}$ ampicillin (SigmaAldrich, Auckland, NZ) supplementation. The transformed cells were cultured for $16 \mathrm{~h}$ at 
$37^{\circ} \mathrm{C}$ before the selection of a positive colony. A single colony for each plasmid was chosen based on normal features of morphology and resistance to ampicillin, and inoculated into $2 \mathrm{~mL}$ sterile liquid LB broth supplemented with $100 \mu \mathrm{g} / \mathrm{mL}$ ampicillin in a $15 \mathrm{~mL}$ Falcon tube and cultured for $16 \mathrm{~h}$ at $37^{\circ} \mathrm{C}$ with constant shaking (Bioline Shaking Incubator, Edwards Instrument Company, Australia). Sterile liquid LB medium (250 mL) containing $100 \mu \mathrm{g} / \mathrm{mL}$ ampicillin supplementation was inoculated with $500 \mu \mathrm{L}$ of each bacterial culture in $500 \mathrm{ml}$ conical flasks and cultured for $16 \mathrm{~h}$ in a $37^{\circ} \mathrm{C}$ shaking incubator. Bacterial cultures were concentrated via centrifugation in a SORVALL RC 6+ ultrafuge (ThermoScientific) at 5,000 xg for $10 \mathrm{~min}$. The plasmid isolation and purification was carried out using a Hi Speed Plasmid Maxi kit (Qiagen, Australia) following the manufacturer's protocol. Plasmid concentration was assessed using an ND-1000 Nanodrop spectrophotometer (Thermofisher Scientific). The sample quality was assessed by electrophoresis of $2 \mu \mathrm{L}$ of a 1:100 dilution of plasmid sample in a $1 \%$ agarose gel for $1 \mathrm{~h}$ at $100 \mathrm{~V}$ alongside a known high quality plasmid preparation (plasmid species pFA6ahphNT1, (Janke, Magiera et al. 2004)) and a 1kb DNA Ladder (Life Technologies. Fresh glycerol stocks of each bacterial strain were prepared by diluting $500 \mu \mathrm{L}$ of liquid LB grown to bacterial saturation in $500 \mu \mathrm{L}$ sterile glycerol in Cryo. s cryotubes (Greiner Bio-One) and stored at $-80^{\circ} \mathrm{C}$ until additional plasmid preparations were required.

The following figure (Figure 2.2.1) represents the cloned sequence utilised during this investigation. WT human $\beta$ I-tubulin with a contiguous tGFP fusion protein is expressed following transfection of the vector containing this sequence, driven by the highly active cytomegalovirus (CMV) promoter. Within the vector, antibiotic resistance genes Amp and Neo are included to allow clonal selectivity in ampicillin (bacteria) or geneticin (mammalian) respectively. A mutated codon corresponding to amino acid 297 was introduced into this plasmid. 


\section{Cloning schematic for the final RG203629 mutation plasmid}

TTTTGTAATACGACTCACTATAGGGCGGCCGGGAATTCGTCGACTGGATCCGGTACCGAGGAGATCT GCCGCCGCGATCGCC

ATGAGGGAAATCGTGCACATCCAGGCTGGTCAGTGTGGCAACCAGATCGGTGCCAAGTTCTGGGAGG TGATCAGTGATGAACATGGCATCGACCCCACCGGCACCTACCACGGGGACAGCGACCTGCAGCTGGA CCGCATCTCTGTGTACTACAATGAAGCCACAGGTGGCAAATATGTTCCTCGTGCCATCCTGGTGGATC TAGAACCTGGGACCATGGACTCTGTTCGCTCAGGTCCTTTTGGCCAGATCTTTAGACCAGACAACTTT GTATTTGGTCGTCTGGGGCAGGTAACAACTGGGCCAAAGGCCACTACACAGAGGGCGCCGAGCTGGT TGATTCTGTCCTGGATGTGGTACGGAAGGAGGCAGAGAGCTGTGACTGCCTGCAGGGCTTCCAGCTG ACCCACTCACTGGGCGGGGGCACAGGCTCTGGAATGGGCACTCTCCTTATCAGCAAGATCCGAGAAG AATACCCTGATCGCATCATGAATACCTTCAGTGTGGTGCCTTCACCCAAAGTGTCTGACACCGTGGTC GAGCCCTACAATGCCACCCTCTCCGTCCATCAGTTGGTAGAGAATACTGATGAGACCTATTGCATTGA CAACGAGGCCCTCTATGATATCTGCTTCCGCACTCTGAAGCTGACCACACCAACCTACGGGGATCTG AACCACCTTGTCTCAGCCACCATGAGTGGTGTCACCACCTGCCTCCGTTTCCCTGGCCAGCTCAATGC TGACCTCCGCAAGTTGGCAGTCAACATGGTCCCCTTCCCACGTCTCCATTTCTTTATGCCTGGCTTTGC CCCTCTCACCAGCCGTGGAAGCCAGCAGTATCGAGCTCTCACAGTGCCGGAACTCACCCAGCAGGTC TTCGATGCCAAGAACATGATGGCTGCCTGTGACCCCCGCCACGGCCGATACCTCACCGTGGCTGCTGT CTTCCGTGGTCGGATGTCCATGAAGGAGGTCGATGAGCAGATGCTTAACGTGCAGAACAAGAACAGC AGCTACTTTGTGGAATGGATCCCCAACAATGTCAAGACAGCCGTCTGTGACATCCCACCTCGTGGCCT CAAGATGGCAGTCACCTTCATTGGCAATAGCACAGCCATCCAGGAGCTCTTCAAGCGCATCTCGGAG CAGTTCACTGCCATGTTCCGCCGGAAGGCCTTCCTCCACTGGTACACAGGCGAGGGCATGGACGAGA TGGAGTTCACCGAGGCTGAGAGCAACATGAACGACCTCGTCTCTGAGTATCAGCAGTACCAGGATGC CACCGCAGAAGAGGAGGAGGATTTCGGTGAGGAGGCCGAAGAGGAGGCC ACGCGTACGCGGCCGCTCGAG - tGFP Tag -----Vector Backbone

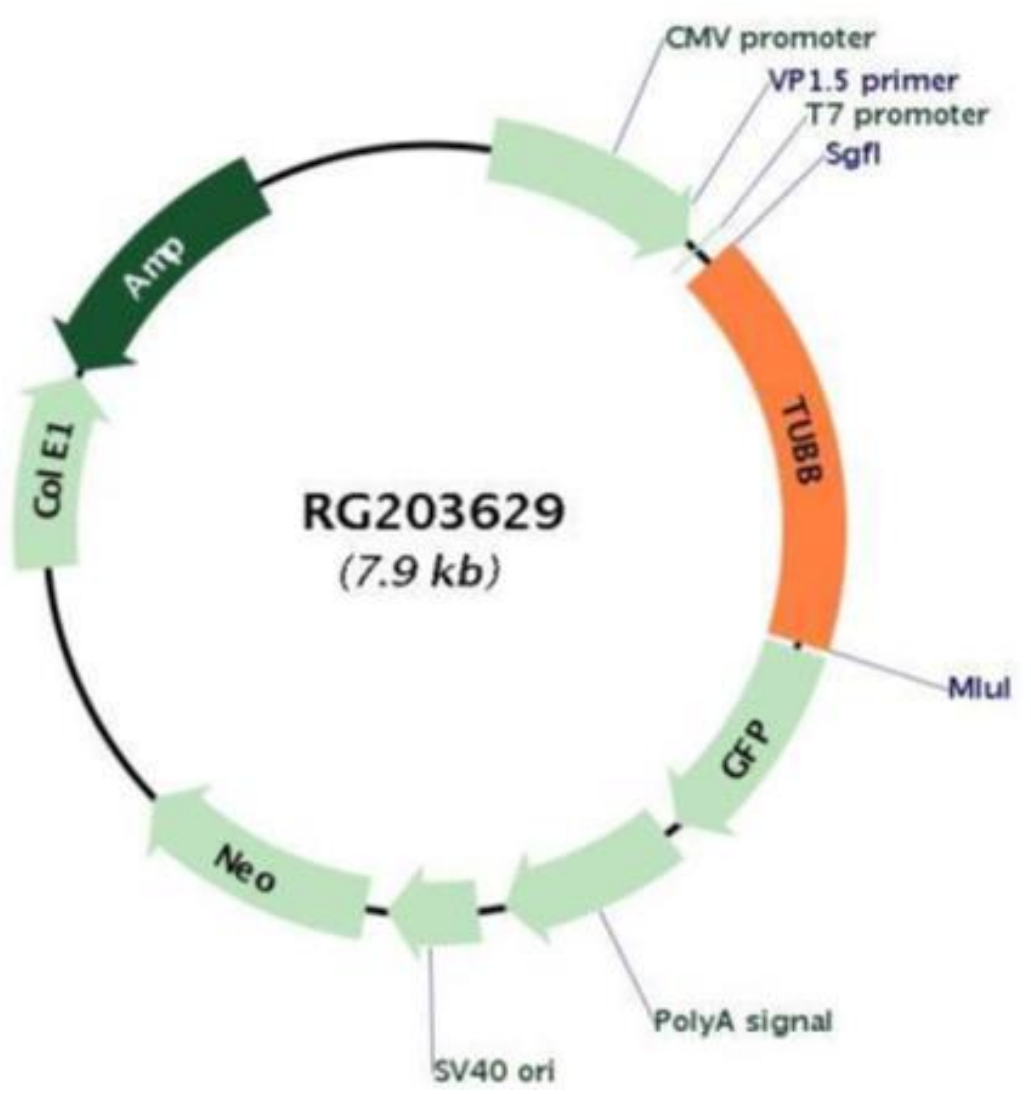

Figure 2.2.1 Cloning schematic for mutation plasmid. Human $\beta \mathrm{I}-$ tubulin sequence within the multiple cloning region of The PrecisionShuttle mammalian vector with C-terminal TurboGFP flag pCMV6-AC-GFP (Origene). Genomic sequence encoding human $\beta 1$-tubulin is indicated in purple. Sequence indicated in orange corresponds to sites of restriction enzyme digest by SgF 1 and Mlu 1 used in cloning $\beta 1$-tubulin sequence into the cassette. Sequence indicated in green corresponds to the fluorescent tGFP fusion tag expressed contiguously with the mammalian $\beta 1$ tubulin product. Sequence in black represents vector backbone DNA as presented in the visual schematic. 


\subsubsection{Transfection optimisation of HEK293}

HEK 293 cells were seeded in 12 -well plates at $2 \times 10^{5}$ cells per well in $1.0 \mathrm{~mL}$ growth medium and allowed to adhere overnight. At approximately $75-80 \%$ confluence, growth medium was replaced with $900 \mu \mathrm{L}$ antibiotic-free growth medium and transfection with varied quantities of tGFP- $\beta 1$-tubulin expression plasmid and Lipofectamine $3000^{\circledR}$ reagent (Invitrogen) was carried out to determine optimal transfection conditions. The quantity of plasmid DNA for transfection was assessed multiple times before optimisation over a range of $0.6 \mu \mathrm{g}-1.0 \mu \mathrm{g}$ per well by diluting tGFP- $\beta$ I-tubulin plasmid DNA with P3000 ${ }^{\circledR}$ reagent (Invitrogen) in a 1:2 ratio ( $\mu \mathrm{g}$ DNA: $\mu \mathrm{L}$ P3000 $\left.{ }^{\circledR}\right)$. The mixtures were then diluted up to a final volume of $50 \mu \mathrm{l}$ in Optimem Reduced Serum Medium (ORSM) (Invitrogen) per transfection mixture. The optimum quantity of Lipofectamine $3000^{\circledR}$ reagent was assessed over the plasmid to reagent ratios $1: 1,1: 2$ and $1: 4 \mu \mathrm{L}$ reagent to $\mu \mathrm{g}$ DNA by diluting the Lipofectamine $3000^{\circledR}$ in a second tube with a final volume of $50 \mu$ l ORSM. After a 5 min incubation at room temperature, the diluted Lipofectamine $3000^{\circledR}$ was mixed gently with the appropriate DNA: $\mathrm{P} 3000^{\circledR}$ complex containing tube and incubated for $20 \mathrm{~min}$ at room temperature to allow complete formation of cationic-lipid complexes. The transfection mixture $(100 \mu \mathrm{L})$ was then added sequentially to each appropriate transfection well giving a final volume of $1 \mathrm{~mL}$ transfection medium. Cells were then grown for $48 \mathrm{~h}$, and optimal transfection conditions were evaluated by examination of GFP expression and cell viability using a fluorescence microscope (Olympus IXS1, Olympus, Tokyo, Japan).

\subsubsection{Transfection of HEK293 for experimental applications}

HEK 293 cells were seeded in 6-well plates at $5 \times 10^{5}$ cells per well in $1.5 \mathrm{~mL}$ growth media and allowed to adhere overnight. At approximately $75-80 \%$ confluence, media was replaced with $1.25 \mathrm{~mL}$ antibiotic-free growth media and transfection was carried out with 
a scaled up version of the optimal protocol determined in section 2.2.4. Specifically, $2 \mu \mathrm{g}$ per well of tGFP- $\beta$ I-tubulin or tGFP-D297I- $\beta$ I-tubulin plasmid were added to independent reaction mixtures before the addition of $4 \mu \mathrm{L} P 3000^{\circledR}$ reagent per well. The combined plasmid DNA and $\mathrm{P} 3000^{\circledR}$ solution was then diluted to a final volume of $125 \mu \mathrm{L}$ per well in ORSM. A second tube was prepared with $4 \mu \mathrm{L}$ Lipofectamine $3000^{\circledR}$ per well diluted to a final volume of $125 \mu \mathrm{L}$ in ORSM. After a $5 \mathrm{~min}$ incubation at room temperature, $125 \mu \mathrm{L}$ per well of dilute Lipofectamine $3000^{\circledR}$ was gently mixed with each plasmid $+\mathrm{P} 3000^{\circledR}$ tube and incubated for $20 \mathrm{~min}$ at room temperature. The final volume of $250 \mu \mathrm{L}$ per well of complete transfection complex was then added to each well giving a total volume of 1.5 $\mathrm{ml}$ transfection medium within each transfection well in the 6-well plate. Cells were then grown for $48 \mathrm{~h}$ before use to allow exogenous expression levels to reach their maximum. Transfection efficiency and cell health were assessed by fluorescence microscopy (Olympus IXS1, Olympus, Tokyo, Japan) by imaging the presence of tGFP signal under fluorescence and the total cell count under phase-contrast using the $10 \mathrm{x}$ objective. Selection using geneticin (G418) was avoided, as preliminary experiments in transfected cells expressing the aminoglycoside 3'-phosphotransferase APH3' II, exhibited inconsistent responses in response to antibiotic selection, impacting overall cell health and introducing changes in sensitivity to microtubule-stabilisers. Instead of selection in G418, the high efficiency transfection protocol described in section 2.2.4 was used to optimise the procedure, maintain the health of cells and minimise the proportion of non-transfected cells present in the cultures.

\subsubsection{Confirmation of transfection by fluorescence microscopy}

Forty-eight hours following transfection, cells were imaged by fluorescence microscopy before treatment with microtubule-stabilising agents in downstream experiments. The same transfected cultures were again imaged at $144 \mathrm{~h}$ post-transfection. These images were 
examined to determine both the transfection efficiency and stability of transgene expression within the whole population of cells over time course of the concentration-response studies. Six-well plates were imaged using the $10 \mathrm{x}$ objective on a fluorescence microscope (Olympus IXS1, Olympus, Tokyo, Japan). An area representative of the sample was imaged under the FITC filter upon exposure of the cells to a mercury lamp excitation source. An exposure time that limited the visibility of non-transfected cells or debris was used to aid in keeping images comparable across transfections. The same field of view was then imaged under phase contrast light microscopy. The two images were overlaid using ImageJ software (NIH), with the green channel threshold set above background fluorescence. Due to the relatively high transfection efficiency obtained by optimisation of the protocol as described in section 2.2.4, further quantitative analysis of transfection efficiency by flow cytometry was not necessary as tGFP reporter activity was highly consistent and widespread throughout the transfected population. A randomised region within each image of an equivalent size was selected, with care taken to include at least 100 evenly distributed cells. These images were then quantified via the CellCounter plugin for ImageJ with the total cell count, and fluorescent cell count recorded to calculate the efficacy of transfection.

\subsubsection{Confirmation of exogenous tubulin expression by western blotting}

Cell samples of transfected 1A9 or HEK293 cells were prepared to correspond to both the $48 \mathrm{~h}$ and $144 \mathrm{~h}$ time points of the MTT cellular proliferation assay described in section 3.2.1. Whole cell samples were collected from cell suspensions by centrifugation of $2 \times 10^{6}$ cells at $400 \mathrm{~g}$ for $5 \mathrm{~min}$ in $1.5 \mathrm{ml}$ Eppendorf tubes before aspiration of residual growth medium. Cell pellets were then stored immediately at $-80^{\circ} \mathrm{C}$ for later use in expression blotting experiments. Cell lysates were prepared from complete sets of cell samples by lysis in $60 \mu \mathrm{L}$ RIPA buffer ( $25 \mathrm{mM}$ TRIS-HCl, $150 \mathrm{mM} \mathrm{NaCl}, 5 \mathrm{mM}$ EDTA, .1 \% SDS, $1 \%$ Triton X-100, $1 \%$ sodium deoxycholate) containing $10 \mu \mathrm{L} / \mathrm{mL}$ Protease Inhibitor Cocktail 
I (Sigma-Aldrich, Auckland, NZ). Sample pellets were suspended by brief vortex mixing, then placed on an orbital shaker for $30 \mathrm{~min}$ at $4^{\circ} \mathrm{C}$ to ensure complete lysis and suspension of total cellular protein. Samples were then centrifuged at $14,000 \mathrm{~g}$ for $20 \mathrm{~min}$ at $4^{\circ} \mathrm{C}$ after which the supernatant containing the proteins of interest was transferred to a new $1.5 \mathrm{~mL}$ Eppendorf tube and the pelleted debris discarded. All samples were kept on ice for the subsequent steps. Protein quantification was carried out using the BCA protein assay (Thermofisher) following the manufacturer's instructions. Samples were prepared for separation by denaturing SDS-PAGE by diluting $30 \mu \mathrm{g}$ of lysate protein in $1 \mathrm{x}$ Laemmli buffer containing $10 \% \beta$-mercaptoethanol and Milli-Q water to a final volume of $20 \mu \mathrm{L}$. A $1.5 \mathrm{~mm} 10 \%$ polyacrylamide resolving gel with a $6 \%$ polyacrylamide stacking gel was loaded with $20 \mu \mathrm{L}$ of prepared lysate sample per lane including $1.5 \mu \mathrm{L}$ molecular weight ladder (Dual Colour Precision Plus ${ }^{\mathrm{TM}}$, Bio-Rad Laboratories) to assist in determining the identity of proteins of interest. Surplus cell lysates were stored at $-80^{\circ} \mathrm{C}$ due to the high stability of the proteins of interest. Electrophoretic separation of the sample proteins was carried out at $120 \mathrm{~V}$ for $1.5 \mathrm{~h}$ or until complete migration of the dye front through the resolving gel had occurred. Proteins were transferred electrophoretically at $4^{\circ} \mathrm{C}$ for $17 \mathrm{~h}$ at a constant $20 \mathrm{~V}$ to a methanol-activated low fluorescence background PVDF membrane (Immobilon-P, Millipore Corp, MA, USA). Following protein transfer, membranes were blocked using 5\% BSA in PBST (1 x PBS with $0.1 \%$ Tween-20 by volume) for $1 \mathrm{~h}$ at room temperature on a rocking platform. Prior to immunological investigation, membranes containing tGFP positive samples were exposed to ambient sunlight for $1 \mathrm{~h}$ as an additional measure to ensure complete exclusion of tGFP emission spectra during fluorescence scanning. Acrylamide gels containing residual proteins were treated for $1 \mathrm{~h}$ at room temperature with a fixative solution of $50 \% \mathrm{EtOH}$ and $3 \%$ ortho-phosphoric acid (by volume) diluted in Milli-Q water. Gels were then washed three times with Milli-Q water 
before submersion in Coomassie staining solution (0.06\% Coomassie® Brilliant blue G250 (Biorad Laboratories, Hercules, CA, USA) dissolved in 34\% methanol, 17\% ammonium sulphate and 3\% phosphoric acid by volume) and left to develop on a rocking platform at room temperature for two days to ensure a complete protein stain before being examined for transfer quality. Conditions for immunological probing of blocked membranes along with individual experiment control parameters are described in detail in the corresponding results sections. All membranes were washed two times between antibody incubations with PBST for $10 \mathrm{~min}$ at room temperature followed by a third wash with $1 \mathrm{x}$ PBS for $10 \mathrm{~min}$ at room temperature. Probed membranes were immediately scanned using a Fujifilm FLA-5100 Fluorescent Image Analyser (Fuji Photo Film Co. Ltd, Tokyo, Japan), and composite images were generated using the MBF-ImageJ software (Rasband, W.S., ImageJ, NIH, Bethesda, Maryland, USA). Image densitometry was carried out to determine the relative density of membrane bands corresponding to proteins of interest by integrating band density areas after adjusting the image threshold values for background fluorescence and normalising against the integrated band density area of the loading control $\beta$-actin.

\subsubsection{Microtubule morphology and confirmation of mutant-tubulin expression by live cell confocal microscopy}

Glass bottomed confocal culture dishes ( $35 \mathrm{~mm}$ diameter) were treated with sterile PBS containing $500 \mu \mathrm{g} / \mathrm{mL}$ poly-D-lysine hydrobromide (Sigma-Aldrich, Auckland, NZ) for 2 h. After coating, the solution in the dishes was aspirated and the dish rinsed twice with sterile PBS and allowed to dry under UV light within a Logic Labconco® Purifier®

Biological Safety Cabinet (Total Lab Systems Ltd, New Zealand). HEK293 cells transfected with mutant tGFP- $\beta$ 1-tubulin expression plasmids as described in section 2.2.5 were seeded at $4 \times 10^{5}$ cells in $1 \mathrm{~mL}$ growth medium into the freshly coated $35 \mathrm{~mm}$ dishes 
and allowed to adhere overnight. Samples were treated with microtubule-stabilising agents Pel A and PTX at $40 \mathrm{nmol} / \mathrm{L}$ for $6 \mathrm{~h}$ to promote microtubule-stabilisation for comparison to control samples. Prior to confocal imaging, the culture supernatant was aspirated and the medium replaced with $1 \mathrm{~mL}$ fresh growth media that contained $200 \mathrm{ng} / \mathrm{mL}$ live cell fluorescent nucleic acid counterstain Hoechst 33342 (639, ImmunoChemistry Technologies, Bloomington, MN, USA). The stain was aspirated after $45 \mathrm{~min}$, and the samples were gently rinsed twice with $1 \mathrm{~mL}$ sterile PBS to remove excess stain before the addition of $1 \mathrm{~mL}$ fresh culture medium, followed by rest for $2 \mathrm{~h}$ before confocal imaging. Confocal images representing cells in various mitotic states in each sample were captured using an Olympus FluoView FV1000 IX81 laser-scanning confocal microscope utilising a $100 \mathrm{X}$ oil immersion objective (1.45 NA) and a humidified stage top incubator with a 5\% $\mathrm{CO}_{2}$ in air atmosphere. This allowed for image acquisition at $37^{\circ} \mathrm{C}$ to prevent aberrant microtubule depolymerisation induced by low temperature. Excitation of the Hoechst 33342 fluorophore used as the nuclear counterstain was achieved using the $405 \mathrm{~nm}$ laser line with emission capture through the dichrome DAPI 425-460 nm filter set. Excitation of the tGFP fluorophore of exogenous $\beta$ I-tubulin was achieved using the 473 laser line with emission capture through the dichrome Alexa Fluor 488 485-545 nm filter set. Images were captured in Z-series optical stacks with $2 \mu \mathrm{m}$ additional image capture above and below the image regions of interest. As photobleaching of tGFP and Hoechst 33342 dyes is significant when utilised in live cell imaging, imaging scans were set at an appropriate thickness to allow rapid acquisition through the sample to gain representative slices depicting microtubule morphology and processes typical throughout the culture. 


\subsection{Results}

\subsubsection{Assessment of preparation quality of tGFP- $\beta$ I-tubulin and tGFP-D297I- $\beta$ I- tubulin vectors}

Isolates of the two expression vectors (WT and D297I mutant) (7.9 kb) were prepared as described in section 2.2.3 and were analysed by agarose gel electrophoresis (Figures 2.3.1 and 2.3.2). The plasmid preparations were run alongside a high quality pFA6a-hphNT1 plasmid preparation (1.84 kilobase pairs) as a control in each of the electrophoreses carried out. The presence of a single, discrete bright band indicates that the tGFP- $\beta$ I-tubulin expression vector had been successfully isolated with high sequence integrity, as indicated by the lack of genomic smearing within the lane. This was comparable to the high quality pFA6a-hphNT1 plasmid preparations run as a comparative control. The plasmid preparations were quantified by spectrophotometry using a Nanodrop spectrophotometer, and the final concentrations were found to be $1.32 \mu \mathrm{g} / \mu \mathrm{L}$ for the tGFP- $\beta \mathrm{I}$-tubulin vector and $0.53 \mu \mathrm{g} / \mu \mathrm{L}$ for the D297I-tGFP- $\beta \mathrm{I}-$ tubulin vector. The A260/A280 and A260/A230 ratios were assessed to determine sample purity. Both plasmid preparations exhibited high A260/A280 and A260/A230 ratios of approximately 1.798 and 2.183 respectively, indicating each was relatively free of protein and solvent contaminants. Plasmid preparations suitable for use in mammalian transfection experiments require high purity as protein or solvent contamination associated with transfected DNA can cause significant toxicity to the target cells. Both preparations were deemed suitable for use in transfection. 


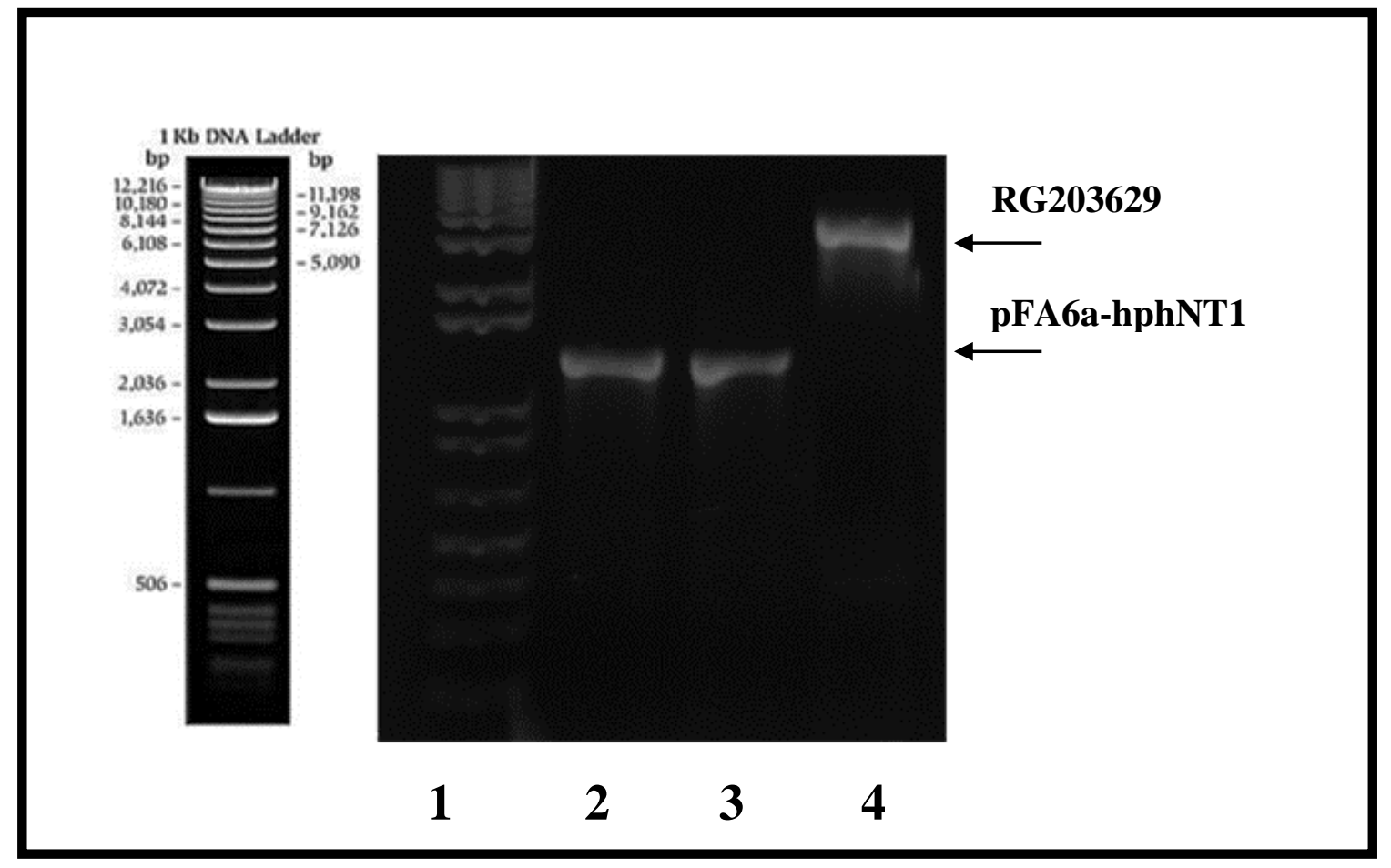

Figure 2.3.1 Agarose gel electrophoresis of tGFP- $\beta 1$-tubulin plasmid. Lane 1: $1 \mathrm{~kb}$ DNA ladder. Lane 2: pFA6a-hphNT1 control plasmid DNA. Lane 3: a 1:10 dilution of pFA6a-hphNT1 control plasmid DNA. Lane 4: tGFP- $\beta 1$-tubulin mammalian expression vector RG203629. 


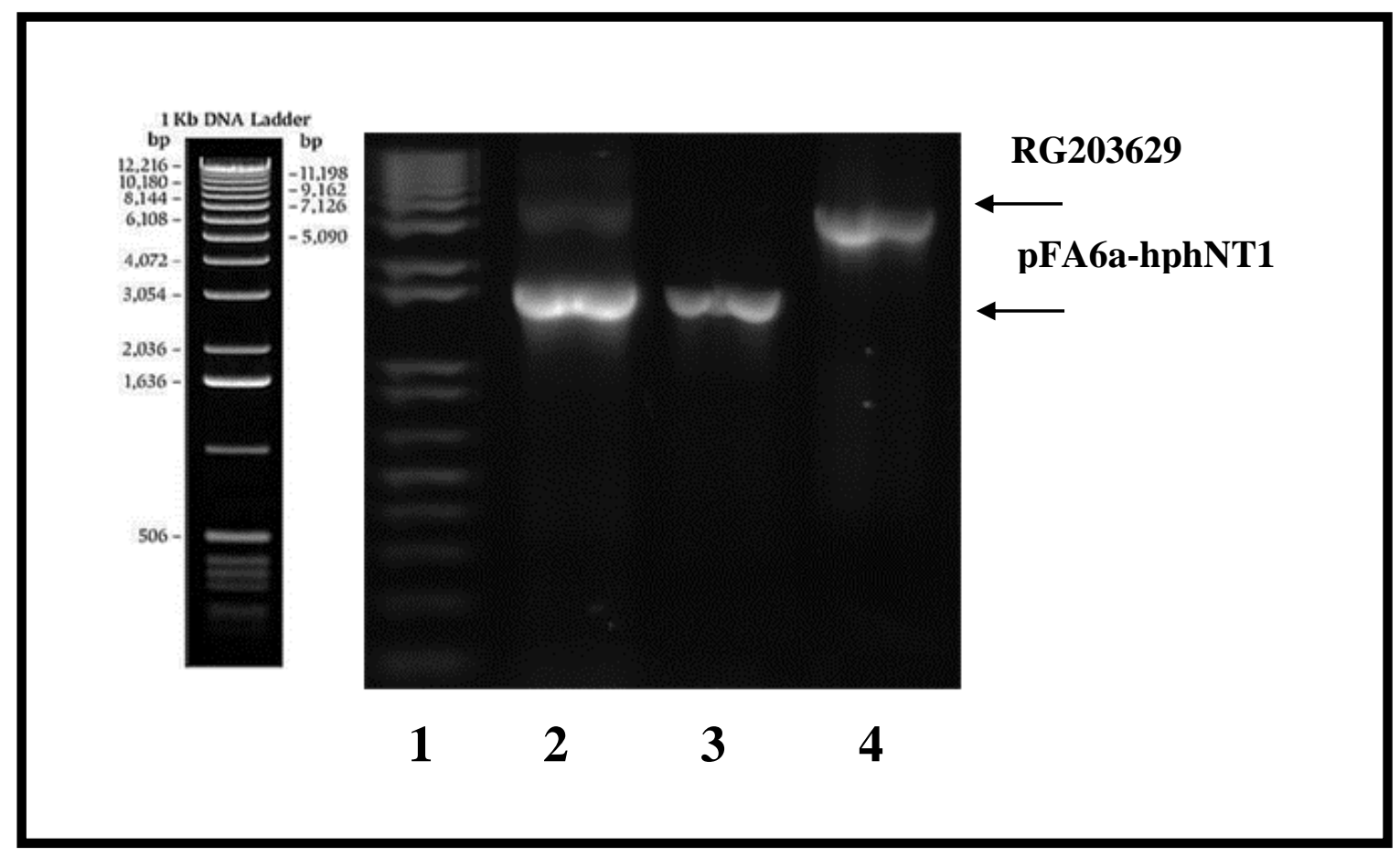

Figure 2.3.2 Agarose gel electrophoresis of $t$ GFP-D297I- $\beta 1$-tubulin plasmid. Lane 1: 1kb DNA ladder. Lane 2: pFA6a-hphNT1 control plasmid DNA. Lane 3: a 1:10 dilution of pFA6ahphNT1 control plasmid DNA. Lane 4: tGFP-D297I- $\beta 1$-tubulin mammalian expression vector D297I-RG203629. 


\subsubsection{Optimisation of transfection in HEK293 cells}

HEK293 cells were transfected with a range of conditions as described in section 2.2.4 until final transfection conditions were identified that resulted in high transgene expression while minimising cell death (Figure 2.3.3). Excessive lipofectamine $3000^{\circledR}$ reagent (greater than $1 \mu \mathrm{L}$ per well) resulted in substantial cell toxicity (approximately $50 \%$ of cells non-viable), similarly to conditions where excess plasmid was detrimental to cell health. Lower plasmid concentrations resulted in fluorescent signals from individual cells similar to transfection with higher plasmid concentration; however, the efficiency of transfection on the total cell population was low (approximately 15-25\% of cells). Selection with G418 was detrimental to the concentration-response of cells in downstream proliferation-assays, whereby large variation in IC50 was observed following selection. This prevented selection in G418 as a means to enrich the portion of the total cell population expressing mutant $\beta$ I-tubulin.

HEK293 cells transfected with $0.8 \mu \mathrm{g}$ plasmid DNA, $1.6 \mu \mathrm{L}$ P3000 ${ }^{\circledR}$ and $1.6 \mu \mathrm{L}$ Lipofectamine $3000^{\circledR}$ reagent exhibited the highest transfection efficiency while maintaining normal cell morphology. As seen in Figure 2.3.3, a lower plasmid concentration resulted in far fewer successfully transfected cells; while higher concentrations up to $1.0 \mu \mathrm{g}$ plasmid DNA were harmful to the cells. The appearance of granular debris and very bright round cells indicates cell death, indicative of this toxicity. 


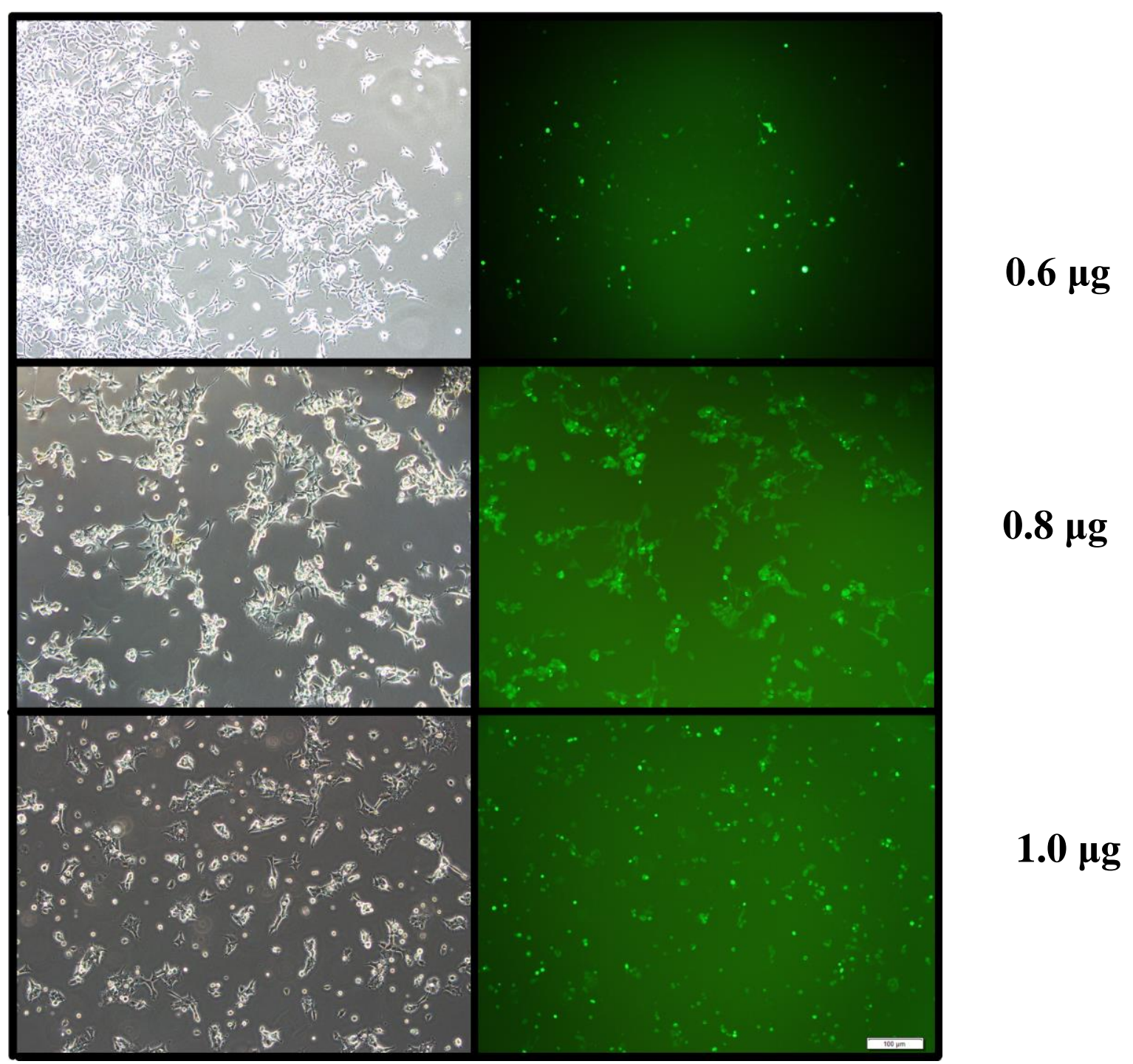

Figure 2.3.3 Assessment of transfection optimisations in HEK293 by fluorescent microscopy. Images are representative of the changes seen across a changing plasmid concentration with 2:1 DNA $\mu \mathrm{g}$ to Lipofectamine $3000^{\circledR}$ in $\mu \mathrm{L}$. Images captured under phase-contrast are shown alongside the same field as viewed through the FITC filter set with tGFP excitation by a mercury lamp. Row 1: HEK293 transfected with $0.6 \mu$ g tGFP- $\beta 1$-tubulin plasmid. Row 2: HEK293 transfected with $0.8 \mu \mathrm{g}$ tGFP- $\beta 1$-tubulin plasmid. Row 3: HEK293 transfected with $1 \mu \mathrm{g}$ tGFP- $\beta 1$-tubulin plasmid. Scale bar $=100 \mu \mathrm{m}$. Note: Problems with imaging equipment at the time of imaging the of $0.6 \mu \mathrm{g}$ plasmid transfection produced the circular exposure. 


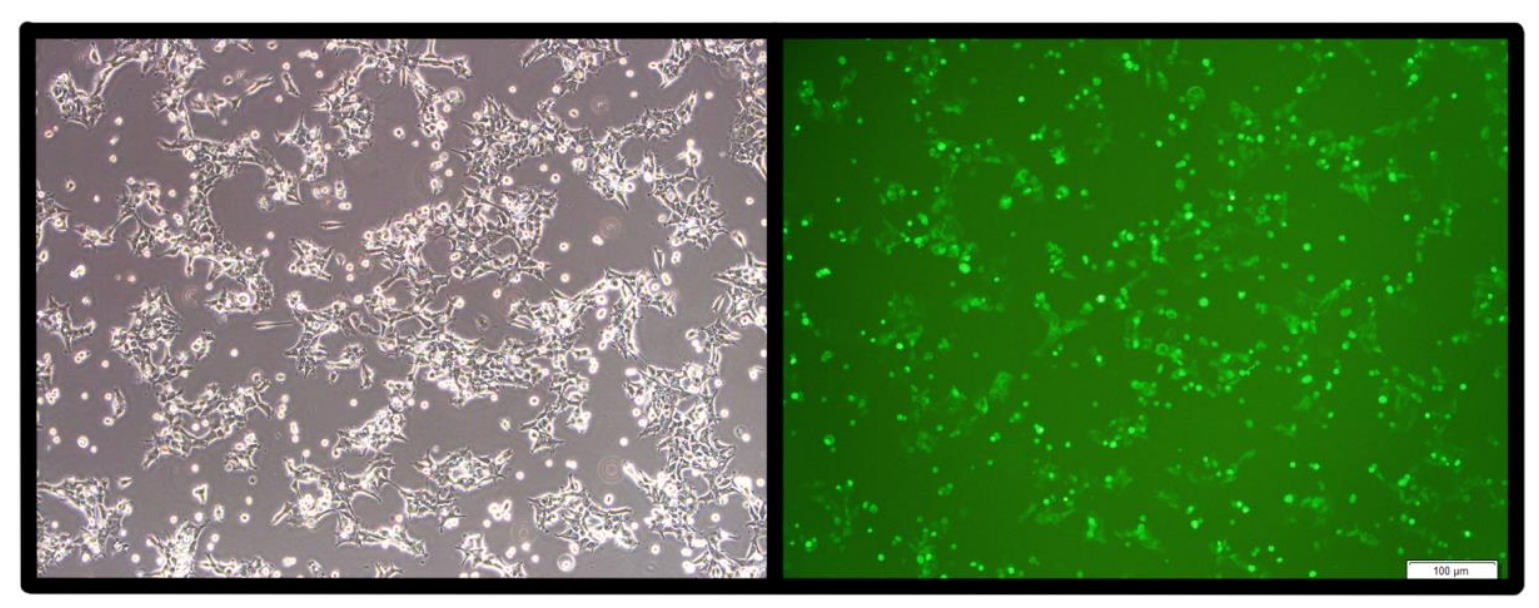

Figure 2.3.4 Further assessment of transfection optimisations in HEK293 by fluorescence microscopy. The image presented is representative of a typical culture with a high concentration of Lipofectamine $3000^{\circledR}$ used during transfection. Shown here is a transfection carried out using 6 $\mu \mathrm{L}$ Lipofectamine and $2 \mu \mathrm{g}$ tGFP- $\beta 1$-tubulin plasmid in a 6 -well format. The image shown was capture under phase-contrast which is shown alongside the same field as viewed through the FITC filter set with tGFP excitation. Scale bar $=100 \mu \mathrm{m}$

HEK293 transfection with higher levels of Lipofectamine $3000^{\circledR}(6 \mu \mathrm{L} /$ well $)$ as seen above in Figure 2.3.4 resulted in a very high number of tGFP- $\beta$ 1-tubulin positive cells; however, a large portion of these cells appeared unhealthy, as indicated by changes in their morphology. tGFP- $\beta 1$-tubulin positive cell debris can also be seen throughout the culture, suggesting cell death has occurred as a result of Lipofectamine $3000^{\circledR}$ toxicity. Note: this transfection was carried out in a scaled up format and is equivalent to a $0.8 \mu \mathrm{g}$ plasmid transfection in the format of Figure 2.3.3. Transfection under these conditions was not appropriate for further experimentation. The final optimised transfection parameters utilised in downstream experiments was equivalent to the $0.8 \mu \mathrm{g} / \mathrm{well}$ conditions as seen in Figure 2.3.3.

\subsubsection{Confirmation of HEK293 transfection by fluorescence microscopy}

Paired phase-contrast/fluorescence images from both $48 \mathrm{~h}$ and $144 \mathrm{~h}$ post-transfection of tGFP- $\beta 1$-tubulin and D297I-tGFP- $\beta 1$-tubulin were used to assess transfection efficiency of the cells. Randomised images were quantified as described in section 2.2.6. Typical cultures transfected under the optimised conditions were assessed by fluorescence 
microscopy (Figure 2.3.3, $.8 \mu \mathrm{g}$ plasmid) to determine a quantitative transfection efficiency (Figure 2.3.5). There were no significant differences by paired student's $t$-test; $\mathrm{P}>.05$ between each of the two time points ( $48 \mathrm{~h}$ and $144 \mathrm{~h}$ ) assessed, or between the two plasmid species, indicating both plasmids performed similarly. Eight biological replicates were assessed, demonstrating a high level of consistency in the efficiency of each transfection.

\section{HEK293 Percent of population expressing tGFP-tubulin}

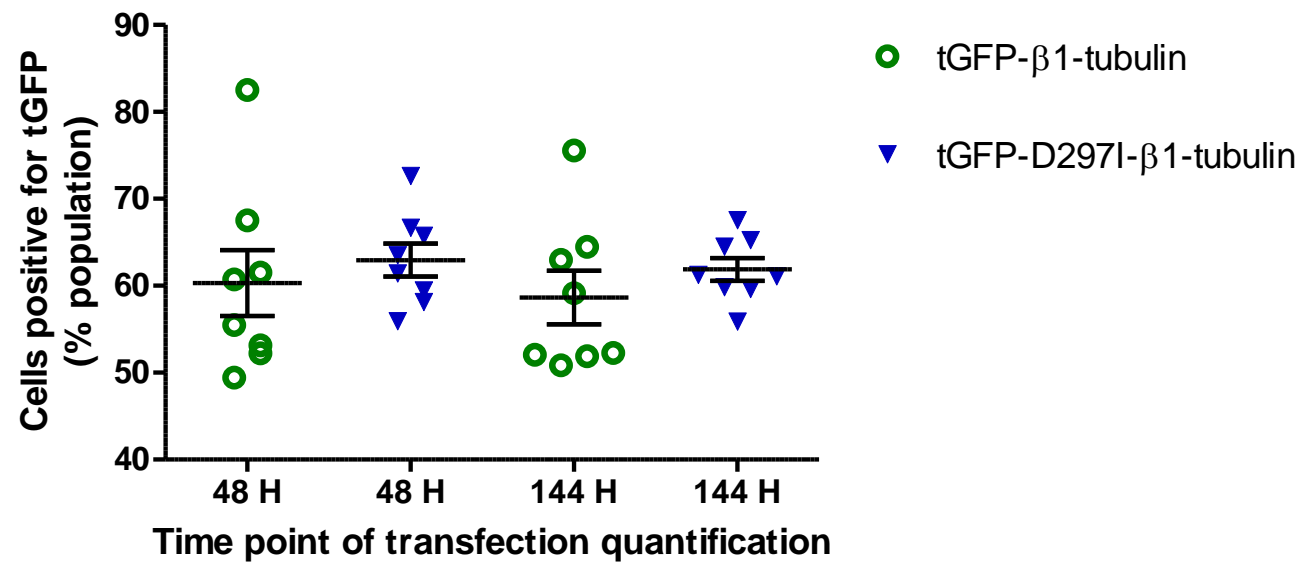

Figure 2.3.5 Quantification of transfection efficiencies by fluorescence microscopy. Samples transfected with either the tGFP- $\beta$-tubulin or tGFP-D297I- $\beta 1$-tubulin expression vectors as described in section 2.2.6 were assessed at $48 \mathrm{~h}$ and again at $144 \mathrm{~h}$ post-transfection to monitor the consistency in the portion of the population positive for exogenous gene expression. Data are presented as the mean \pm SEM of eight biological replicates $(n=8)$.

\subsubsection{Transfection efficiencies in $1 \mathrm{~A} 9$ cells}

1 A9 cells were transfected as described in section 2.2 .5 to assess the suitability of this cell line for determining the D297I point mutations effects on cell proliferation with MSA treatment. Paired phase-contrast/fluorescence images from both $48 \mathrm{~h}$ and $144 \mathrm{~h}$ posttransfection of tGFP- $\beta 1$-tubulin and D297I-tGFP- $\beta 1$-tubulin were used to assess the distribution of exogenous protein expression in the cultures. The 1A9 cell line exhibited a similar transfection profile at $48 \mathrm{~h}$ post-transfection compared to the HEK293 cells; 
however, the same cultures at $144 \mathrm{~h}$ post-transfection demonstrated a significant loss of tGFP signal throughout the population. Optimisation of the transfection conditions for working with 1A9 cells was discontinued due to the loss of expression at the $144 \mathrm{~h}$ time point. This post-transfection time is earlier than what would be required for conducting the MTT cellular proliferation assay (3-day exposure to the microtubule-stabilising agents after an adequate time for adequate exogenous expression).

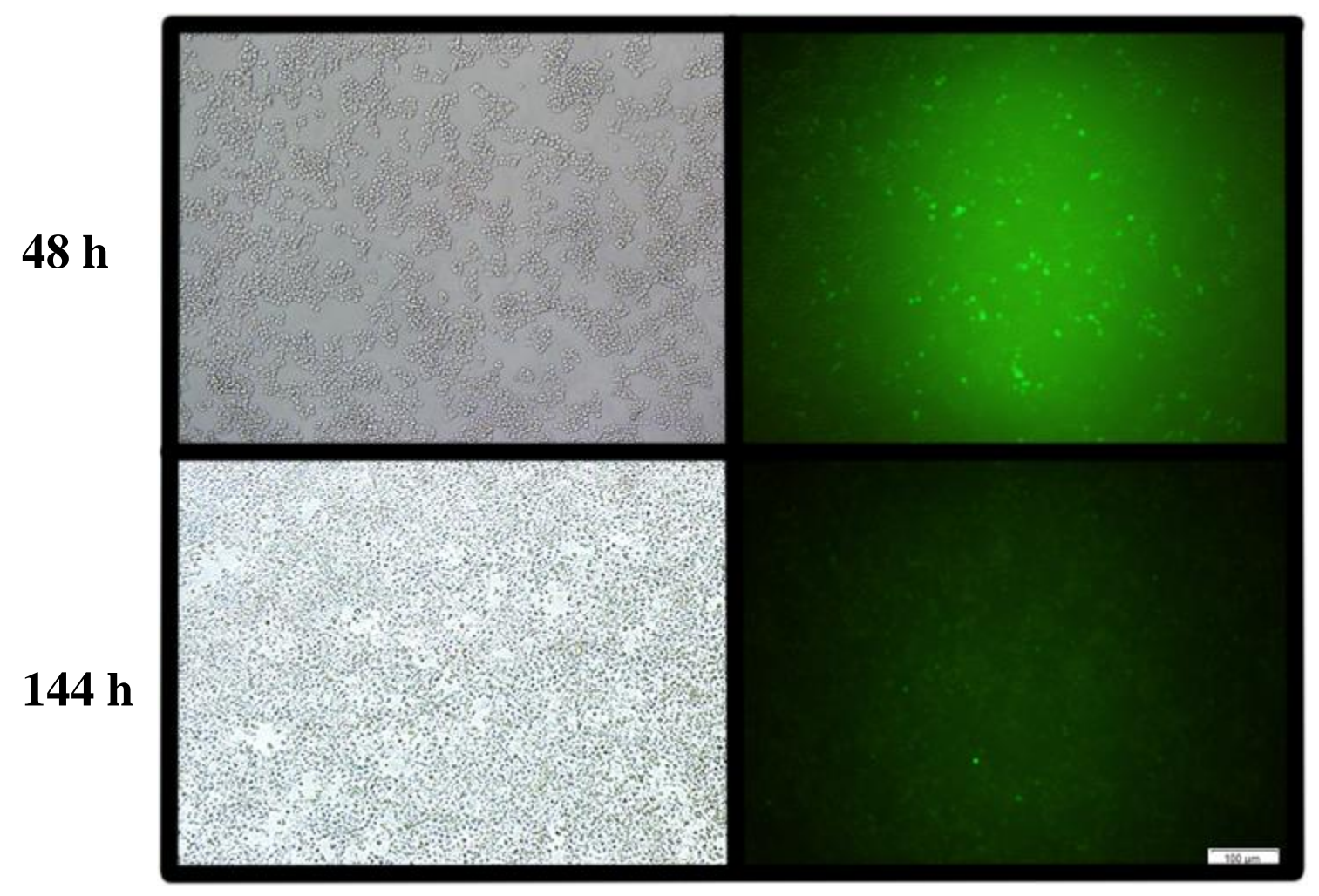

Figure 2.3.6 1 A9 cells transfected with tGFP-D297I- $\beta 1$-tubulin expression vector. Images captured under phase-contrast are shown alongside the same field as viewed through the FITC filter set. Scale bar is indicated in the bottom right frame. Row 1: $1 \mathrm{~A} 9$ cells transfected with $0.8 \mu \mathrm{g}$ tGFP-297I- $\beta 1$ tubulin plasmid at $48 \mathrm{~h}$ post-transfection. Row 2: 1 A9 cells transfected with $0.8 \mu \mathrm{g}$ tGFP-D297I $\beta 1$ tubulin plasmid at $144 \mathrm{~h}$ post-transfection. Note: Transfection conditions were not optimised to the same extent as in HEK293 cells due to the early finding that exogenous expression is lost in 1A9 earlier than that required for cellular proliferation assays to be completed. The bottom right frame demonstrates a widespread loss of the tGFP signal throughout the entire culture. Only rare single cells remain positive for tGFP expression 144h following transfection. Note the 144 hour image appears much darker due to low overall fluorescence in the culture as exposure was kept constant. 


\subsubsection{Confirmation of exogenous tubulin expression by Western blotting:}

Both the exogenous tGFP- $\beta$ I-tubulins (WT and mutant) and the endogenous $\beta$ I-tubulin content of the HEK293 and 1A9 cell lines was assessed by western blotting to determine the relative portion of the total $\beta$ I-tubulin each tGFP fusion construct represented within the transfected cell population. Proteins were prepared for SDS-PAGE as described in section 2.2 .7 before immunological detection. The primary antibody, mouse anti- $\beta$ I-tubulin (T7816, Sigma-Aldrich, Auckland, NZ) was used at a 1:1000 dilution, with goat anti-mouse Alexa Fluor® 488 (A-11001, Life Technologies) at a 1:2000 dilution used as the secondary antibody. For a loading control, mouse anti- $\beta 1$-actin (A2228, Sigma-Aldrich, Auckland, NZ) was used at a 1:1000 dilution with the same secondary antibody.

Cell samples from tGFP- $\beta$ I-tubulin or the point mutant transfected 1 A9 cell cultures displayed an approximate $75 \mathrm{kDa}$ band corresponding to expression of a tGFP tagged tubulin species (Figure 2.3.7). Below these signals, the smaller endogenous $\beta$ I-tubulin band was detected at approximately $50 \mathrm{kDa}$, and the $\beta \mathrm{I}$-actin loading control band at approximately $42 \mathrm{kDa}$. 1A9 samples from the $48 \mathrm{~h}$ post transfection time point displayed abundant tGFP-tubulin species expression with approximately $54 \%$ of the total $\beta I$-tubulin originating from either expression vector. The corresponding $144 \mathrm{~h}$ samples however, did not show detectable levels of tGFP tagged tubulin from either vector indicating a loss of plasmid expression similar to what was seen for 1A9 cells by fluorescence microscopy in Figure 2.3.6. 
A.

\section{$48 \mathrm{~h}$}

$144 \mathrm{~h}$

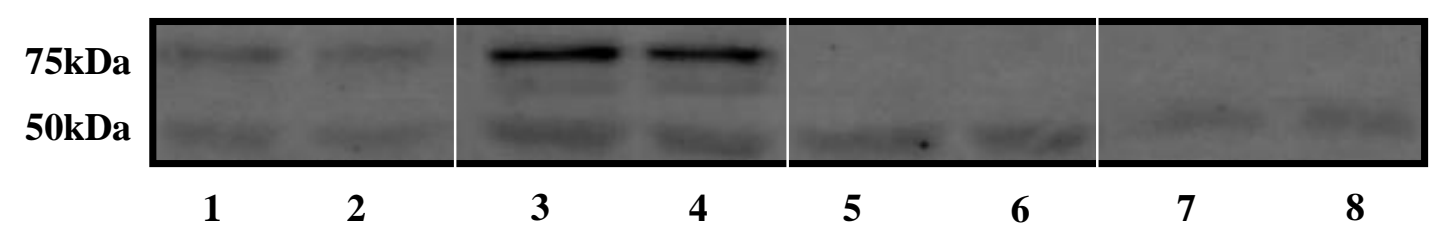

B. Exogenous tubulin expression in $1 \mathrm{~A} 9$

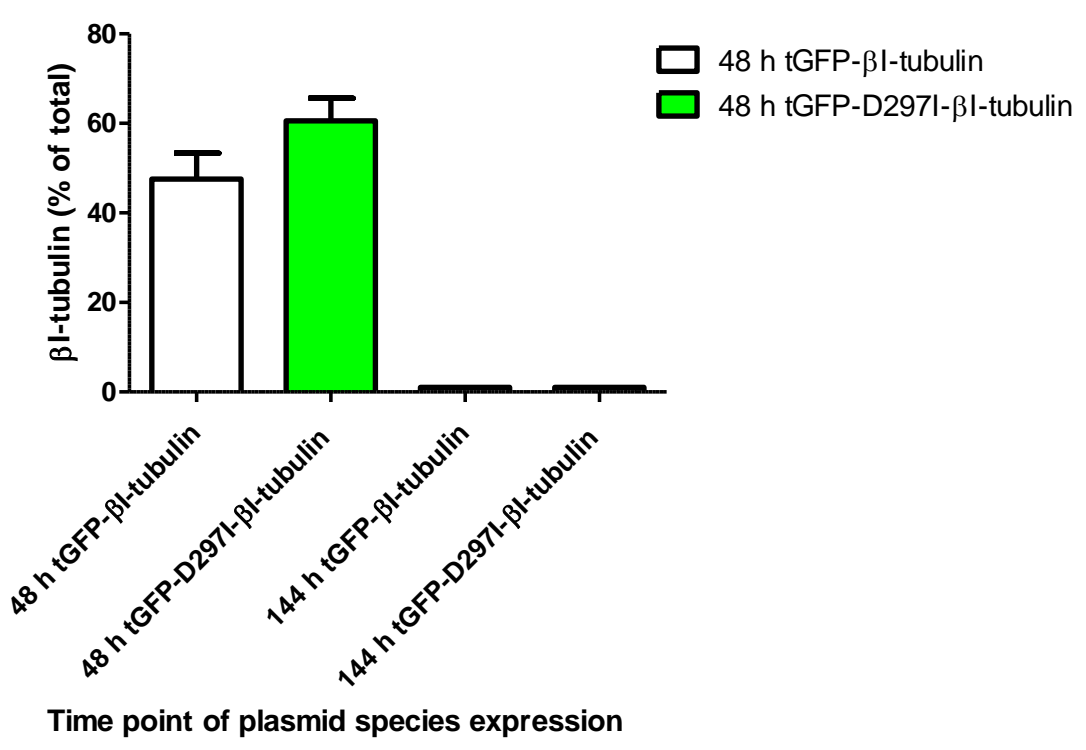

Figure 2.3.7 $\beta$ I-tubulin profile of $1 A 9$ cells samples at $48 \mathrm{~h}$ and $144 \mathrm{~h}$ post-transfection. A) Immunodetection of total $\beta \mathrm{I}$-tubulin in $1 \mathrm{~A} 9$ cells after electrophoretic separation of $30 \mu \mathrm{g}$ protein lysate in a $10 \%$ polyacrylamide gel. The combined image represents the protein bands of interest from two different gels. Lanes 1, 2: samples transfected with tGFP- $\beta 1$-tubulin after $48 \mathrm{~h}$. Lanes 3, 4: samples transfected with tGFP-D297I- $\beta 1$-tubulin after 48 h. Lanes 5, 6: Samples transfected with tGFP- $\beta 1$-tubulin after $144 \mathrm{~h}$. Lanes 7, 8: Samples transfected with tGFP-D297I- $\beta 1$-tubulin after 144 h. At $48 \mathrm{~h}$, both endogenous $\beta \mathrm{I}$-tubulin and the mutant protein product of each expression vector can be seen; however, this same signal at $144 \mathrm{~h}$ post-transfection, no exogenous tubulin bands were detected.

B) Summary of $\beta$ I-tubulin protein abundance data. Densitometry was carried out, and the tubulin band densities were normalised relative to the $\beta$-actin loading control. Only two biological repeats were carried out due to the unsuitable nature of this cell line for further MSA concentration-response studies.

Unlike 1A9, the $\beta$ I-tubulin expression profile of HEK293 cells following transfection did not exhibit this same loss of tGFP- $\beta 1$-tubulin signal after $144 \mathrm{~h}$. These cells maintained their expression of tGFP-tubulin at the $144 \mathrm{~h}$ time point, indicating mutant D297I- $\beta \mathrm{I}-$ tubulin would be present throughout the entire duration of the concentration-response studies required. Expression levels of mutant $\beta \mathrm{I}$-tubulin in transfected HEK293 were 
investigated in much greater depth than in 1A9 cells for these reasons. Mutant $\beta I$-tubulin bands appeared faintly in preliminary HEK293 immunoblotting experiments, indicating a low abundance of exogenous protein expression relative to the total $\beta I$-tubulin pool. Samples were assessed with two different primary antibodies, one targeting the carboxylterminus of $\beta$ I-tubulin (T7816, Sigma-Aldrich, Auckland, NZ), and the other targeting an alternative epitope located at the amino-terminus of $\beta$ I-tubulin $(1: 1000$, AAS61557C rabbit-anti-h $\beta 1$-tubulin, Antibody Verify, Las Vegas, NV). No significant difference in protein abundance was found between the two antibodies, indicating the carboxyl-terminus tGFP-tag on the exogenous $\beta$ I-tubulin species was not likely to be responsible for preventing epitope recognition, thus, leading to the low levels of tGFP-tubulin seen.

\section{D297I- $\beta$ I-tubulin expression in HEK293}

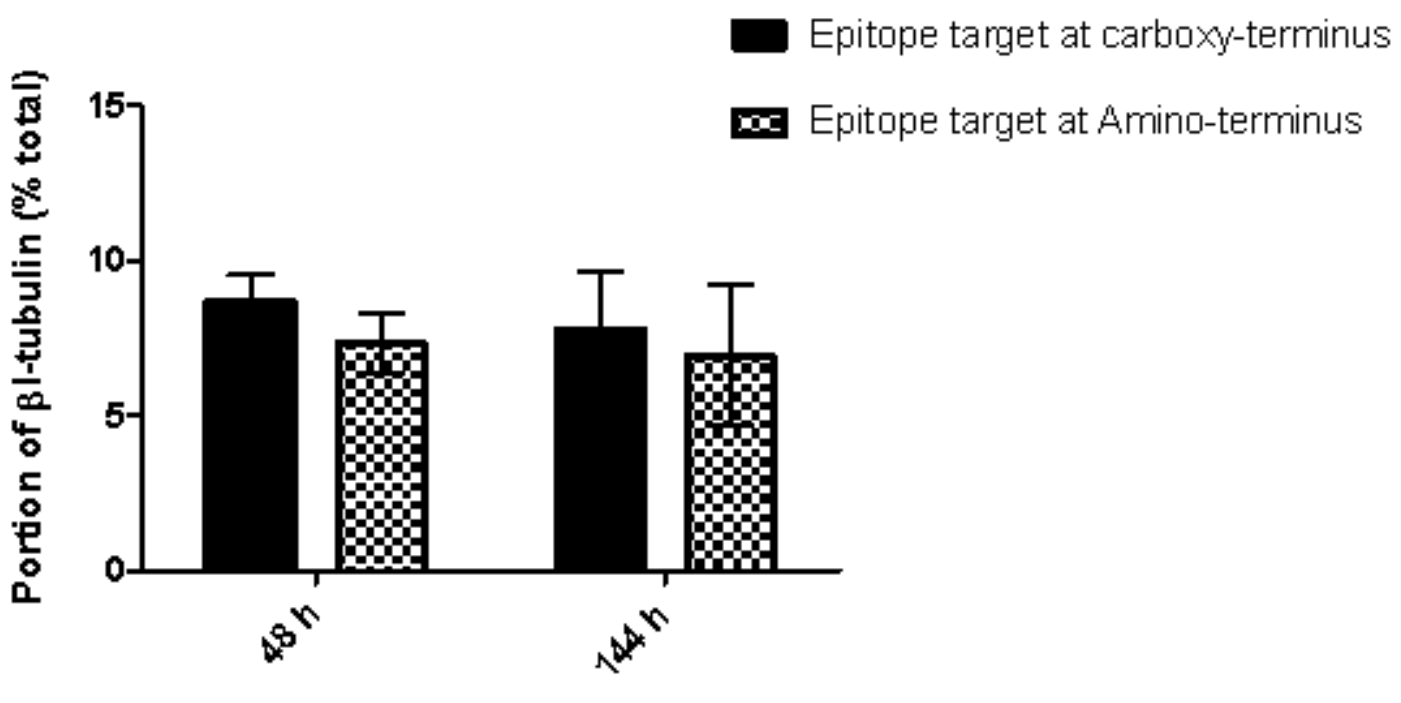

\section{Time of sample quantification (hours post transfection)}

Figure 2.3.8 Detection by antibodies targeting the $C-v s-N$ terminus. Presented is a comparison of tGFP-D297I- $\beta$ I-tubulin expression in HEK293 cells as detected by primary antibodies targeting epitopes located at either the carboxyl-terminus or amino-terminus of $\beta I$-tubulin. Samples taken at both 48 and $144 \mathrm{~h}$ post-transfection showed no significant difference in the level of detectable mutant protein relative to the $\beta$-actin loading control when immunoblotting against either epitope in $\beta I$-tubulin. Data are presented as the mean \pm SEM with no significant difference in densitometry 
Further western blotting was carried out in datasets of 48 and $144 \mathrm{~h}$ samples from paired dose response assays performed as described in 3.2.1 in an attempt to quantify the relative abundance of mutant protein present. As there was no significant difference found between the two primary antibodies, the carboxyl-terminus targeting primary antibody (T7816, Sigma-Aldrich, Auckland, NZ) was used for all future assessments of relative expression. 


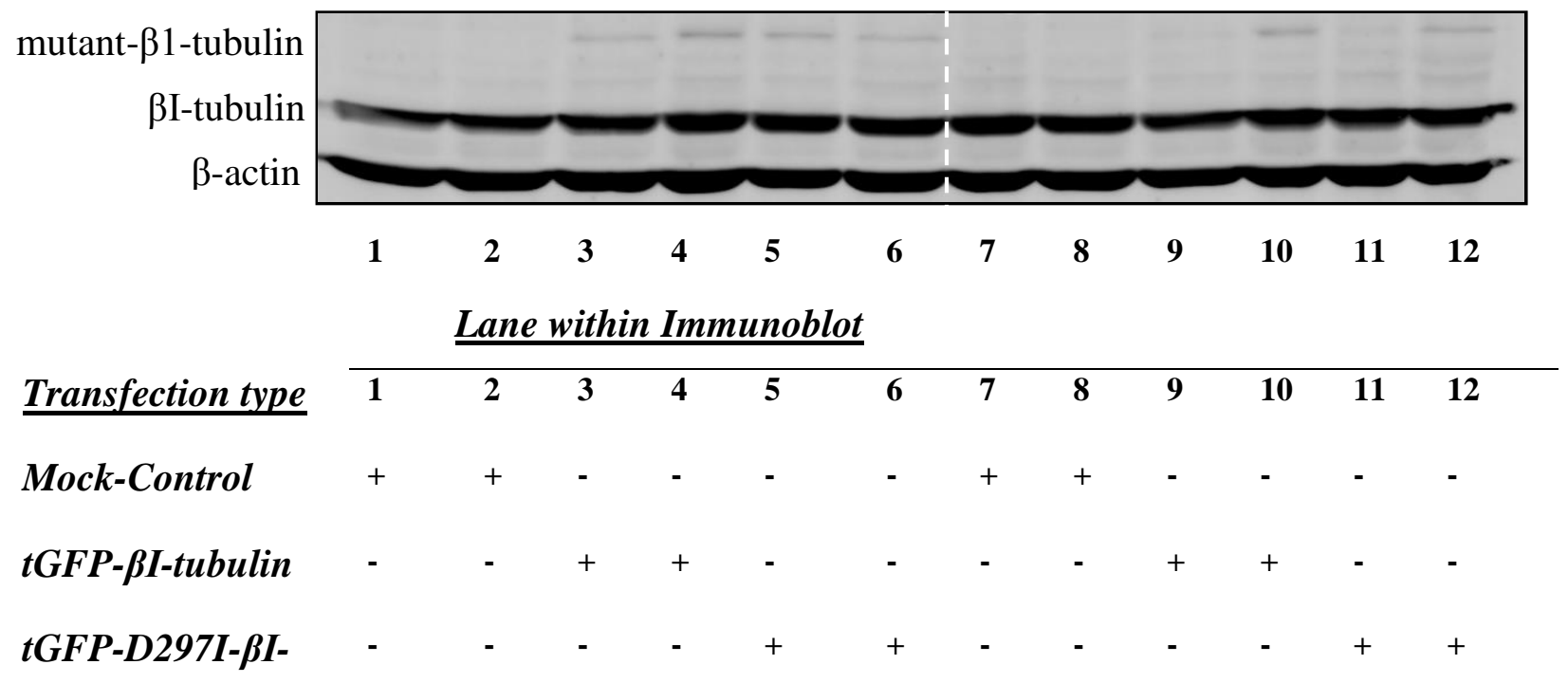

\section{tubulin}

B.

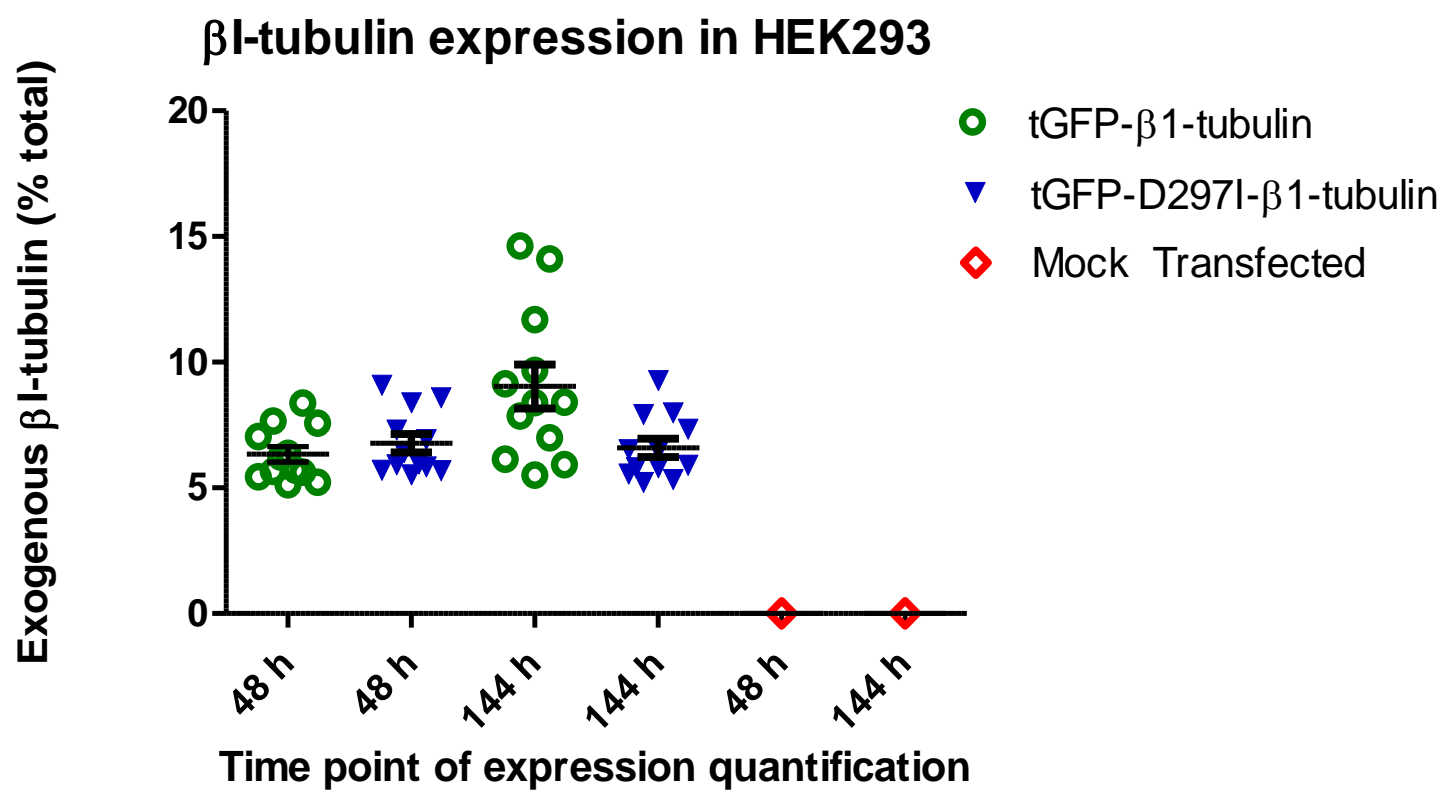

Figure 2.3.9 A) Mutant expression in HEK293 cells. Presented is the $\beta \mathrm{I}$-tubulin profile of samples transfected with mutant $\beta I$ tubulin expression vectors taken at 48 and 144 hours post transfection. Each set of two lanes represent samples taken from the $48 \mathrm{~h}$ followed by the $144 \mathrm{~h}$ time point for a single transfected culture (lanes numbered evenly $=48 \mathrm{~h}$, odd numbers $=144 \mathrm{~h}$ ). This immunoblot represents a typical expression profile from two biological replicates (lanes $1-6$, lanes 7 - 12) with indicated bands corresponding to the mutant tubulin species $(75 \mathrm{kDa})$, endogenous BItubulin $(50 \mathrm{kDa})$ and the $\beta$-actin loading control $(43 \mathrm{kDa})$.

B) Mutant tubulin expression at 48 and $144 \mathrm{~h}$ corresponding to paired concentration-response studies with MSA treatment. Mutant $\beta \mathrm{I}$-tubulin expression in transfected cell samples was assessed for consistency across experiments. Western blotting was performed for 12 biological replicates with samples taken from paired cultures that were used in dose response studies as described in 3.2.1. 
Mutant $\beta I$-tubulin expression was very low compared to the total $\beta$ I-tubulin pool in HEK293 cells (Figure 2.3.9). After accounting for a data skew caused by a single tGFP-

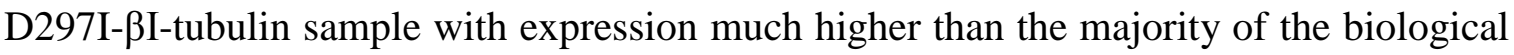
replicates, the level of difference in mutant- $\beta$ I-tubulin expression between samples was not statistically significant. Expression was consistent at both $48 \mathrm{~h}$ and 144h (1-way ANOVA $(\mathrm{p}=.057$ at a $95 \%$ confidence interval $))$.

\subsubsection{Microtubule morphology and conformation of mutant-tubulin expression using live cell confocal microscopy}

The ability of mutant tGFP- $\beta$ I-tubulin subunits to integrate normally into quaternary microtubule structure is critical to understanding the functional significance of the introduced D297I point mutation, or indeed the WT tGFP tagged $\beta$ I tubulin alone. For this reason, the presence of tGFP- $\beta$ I-tubulin in polymerised microtubule structures was assessed by live cell confocal microscopy. HEK293 cells transfected with either of the tGFP- $\beta$ I-tubulin vectors exhibited strong tGFP signals in the confocal microscope (Figure 2.3.9). The appearance of regular, linearized signals within cells was indicative of polymerised microtubule structures, as fusion tagged $\beta$ I-tubulin subunits were incorporated into the quaternary microtubule structure. Cells were observed in different stages of mitosis, with various image slices indicating the presence of bright, condensed mitotic tubulin structures. These condensed structures represent the centrosome, with dense signals corresponding to an abundance of correctly integrated tGFP-fused $\beta$ I-tubulin subunits. Colocalised to these areas, dense nuclear DNA was observed through a strong Hoechst 33342 fluorescence indicating the presence of condensed chromosomal DNA. The presence of these features within the HEK293 cells suggest mutant tGFP- $\beta$ I-tubulin is integrated correctly into polymerised microtubules which maintain a morphology and physiological activity. 
A)
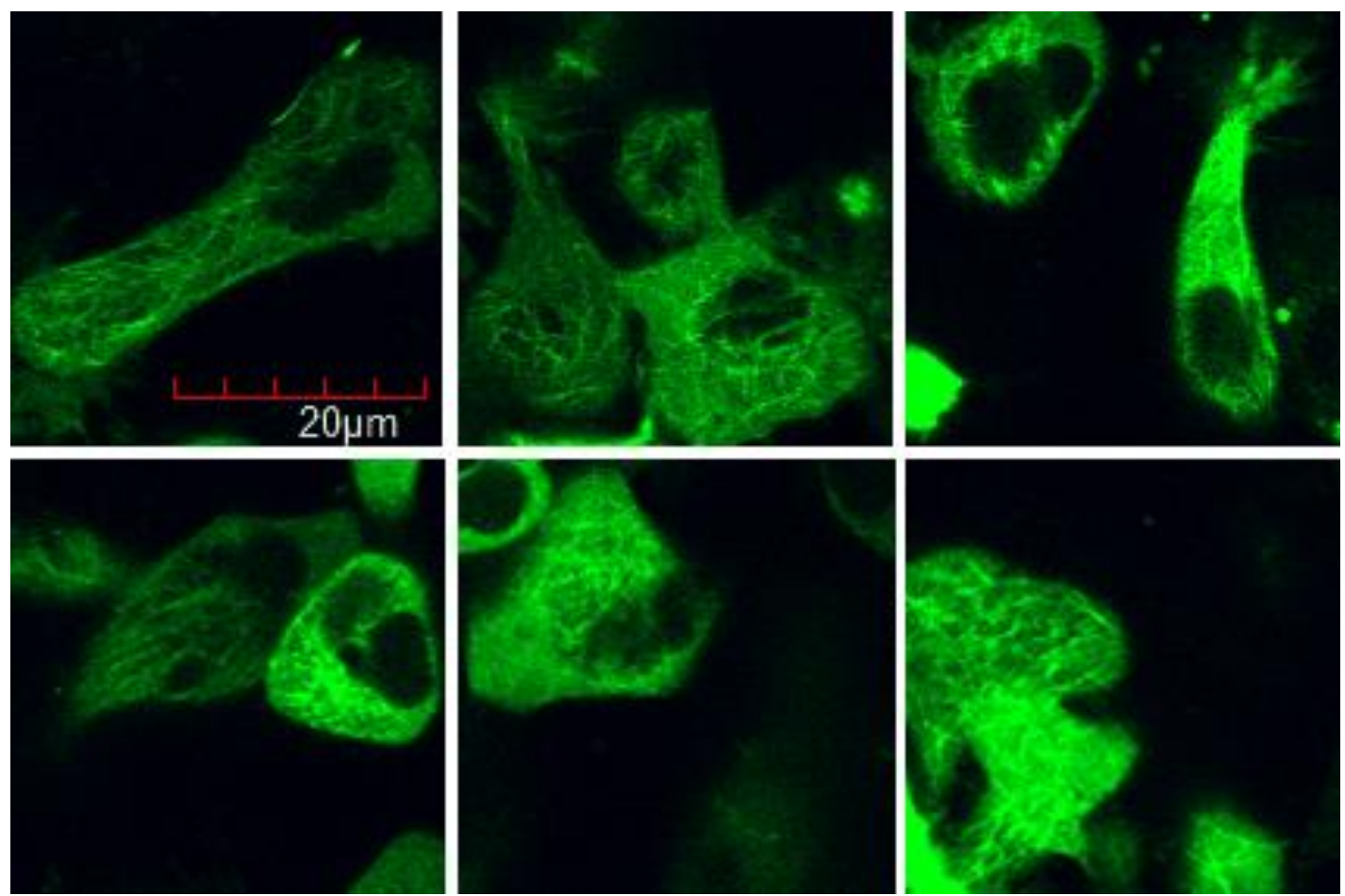

B)
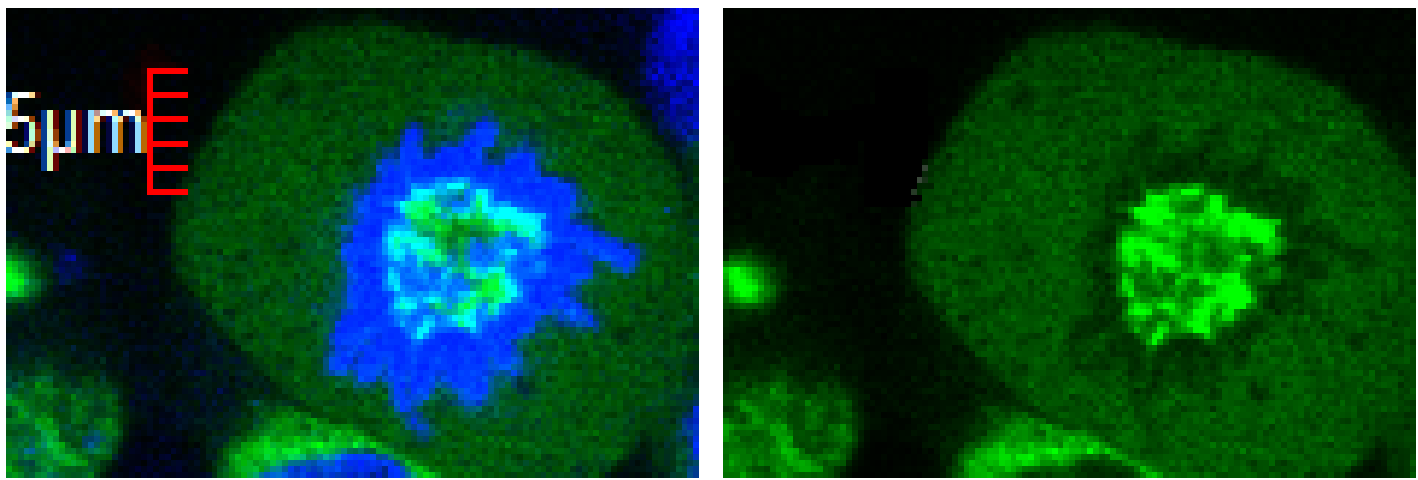

Figure 2.3.10 live cell confocal microscopy A) tGFP-D297I- $\beta I-t u b u l i n$ transfected HEK293 with polymerised microtubules as visualised by confocal microscopy. tGFP- $\beta$ I-tubulin containing microtubules can be clearly seen within individual $\mathrm{z}$-stack slices in various conformations consistent with a normal cellular morphology. Frames presented in A each represent a single optical slice captured from individual cell clusters within a tGFP- $\beta$ I-tubulin expressing culture. Slightly punctate signal distribution is present in frames, suggesting some level of tGFP aggregation. The blue fluorescent nucleoside stain Hoechst 33342 has been omitted for clarity in viewing polymerised microtubule structures. All images in these frames are to scale with the scale bar $(20 \mu \mathrm{m})$ in frame A.

B) $t$ GFP-D297I- $\beta$ I-tubulin transfected HEK293 in an end-on view of prometa phase in cell division. Dense microtubule structures with integrated tGFP-tagged $\beta$ I tubulin are visualised alongside condensed chromosomal structures (blue) as a part of the centrosome complex during cell division. The frame shown on the right depicts a cell with the signal from condensed chromosomal structures removed for clarity. Both images are to scale with the scale bar $(5 \mu \mathrm{m})$ presented in the left-hand frame. 
Cells transfected with tGFP- $\beta$ I-tubulin appear to express considerable amounts of exogenous $\beta \mathrm{I}$-tubulin; however, quantification by confocal microscopy is not possible from these images. While very low levels of expression of mutant $\beta$ I-tubulins were detected from whole cell populations by Western blotting (Figure 2.3.9), mutant $\beta$ I-tubulin within individual successfully transfected cells appears as strong tGFP signals evident throughout the images captured by confocal microscopy.

\subsection{Discussion}

\section{Expression of tGFP-D297I- $\beta I-t u b u l i n$}

The research presented within chapter two of this investigation focused around the introduction of a D297I-mutant- $\beta$ I-tubulin isoform with regular biological function into a mammalian cell model. In order to assess the biological significance of the hydrogenbonding interaction between the $\beta \mathrm{I}-\mathrm{D} 297$ amino acid residue and Pel A or Lau, as predicted by x-ray crystallography (Prota, Bargsten et al. 2014), our mutant construct needed to be assessed for suitability in this investigation. tGFP-D297I- $\beta$ I-tubulin subunits present within transfected cultures, under ideal conditions, would represent the entire $\beta I$-tubulin pool of the cells. In this scenario, any change in the molecular interaction of Pel A or Lau with the D297I mutant residue would be revealed through large shifts in the proliferative drug response phenotype of the cells.

Transfection of the 1A9 cell line with the D297I- $\beta$ I mutation construct provided widespread transgene expression throughout the cell population, even without significant optimisation of the transfection parameters. tGFP-D297I- $\beta$ I-tubulin expression was robust, with a high abundance of mutant tubulin in Western blots by $48 \mathrm{~h}$. Unfortunately, transfected $1 \mathrm{~A} 9$ cells also demonstrated an almost complete loss of this mutant expression in both Western blot and immunocytochemistry studies $144 \mathrm{~h}$ following transfection. This indicated that mutant 
$\beta I$-tubulin subunits were unlikely to be present over the complete time course of any concentration-response experiments for $\mathrm{IC}_{50}$ estimation. Stability in the level of exogenous $\beta I$-tubulins present within a cell population is key to interpretation in an in vitro model, if a good correlation is to be made between mutant tubulin levels and $\mathrm{IC}_{50}$ values.

As a result of the early loss of expression, 1A9 cells were unsuitable for concentrationresponse studies despite the high abundance of mutant $\beta$ I-tubulin present early on. Like 1A9, the HEK293 cell line also demonstrated widespread expression of transfected mutant and WT tubulin; however, unlike 1A9 cells, the transfected HEK293 cultures maintained their expression of tGFP-D297I- $\beta$ I for $144 \mathrm{~h}$, indicating this cell line would be more suitable for use in the in vitro model. Surprisingly, however, the fraction of the $\beta I$-tubulin pool made up by mutant- $\beta$ I subunits was found to be substantially lower than that found in the 1A9 cell line by Western blotting. A lower abundance of mutant $\beta$ I-tubulin expression would certainly reduce the magnitude of any potential changes in proliferative activity in response to Pel A or Lau, seen as the direct result of introducing the D297I-mutant- $\beta$ Itubulin.

In earlier studies of Pel A and Lau binding associations, a sub-clone of the 1A9 cell line (L4) was generated in Dr Paraskevi Giannakkakou's lab at Weil Cornell Medical College through long-term selection in the presence of Lau (Kanakkanthara, Wilmes et al. 2011). This cell line demonstrated a 39-fold increase in resistance to both Lau and Pel A compared to the parental 1A9 cell line. Point mutations R306H or R306C in the $\beta$ I-tubulin amino acid sequence within clones of the L4 cell line implicated this region's importance in the drugprotein association of Lau and Pel A, providing the first mammalian cell-based evidence for the $\beta$ I-tubulin-binding site. A second 1 A9 clone (R1) generated similarly by long-term culture with increasing exposure to Pel A exhibited the point mutation A296T within the 
$\beta I$-tubulin amino acid sequence, conferring a 6-fold resistance to Pel A with no notable alteration in Lau sensitivity. The resistance to Lau and Pel A,f1 especially in the L4 clone, is a prime example of the large shift in concentration-response a single point mutation can confer when the mutation is present within the whole $\beta I$-tubulin complement of the cell population.

In the present study, transfected HEK293 cells expressing mutant $\beta$ I-tubulin demonstrated only a small portion of mutant relative to endogenous $\beta$ I-tubulin content; however, previous studies on the effect of $\beta$ I-tubulin point mutations on PTX resistance found differential resistance to PTX with approximately 5\% exogenous $\beta$ I-tubulin expression (Wang, Yin et al. 2006). The magnitude of these changes was expected to be significantly lower than the 39 fold difference seen in the L4 cells. This is not surprising, given the relatively low tGFP$\beta I$-tubulin abundance in the cells.

Approximately $60 \%$ of HEK 293 cells within the population expressed mutant $\beta I$-tubulin, with a high consistency in expression, albeit at very low abundance based on Westerm blot quantification. It was decided however that because of the stable abundance of the mutant tubulin over the necessary $144 \mathrm{~h}$ time course for an $\mathrm{IC}_{50}$ determination; the HEK293 cell line was suitable for investigation of the importance of the D297 amino acid residue in Pel A or Lau binding interactions. Even a small change in concentration-response resulting from the presence of this mutation would possibly be detected in MTT-proliferation data due to the highly consistent behaviour in aspects of transfection, expression and proliferation seen in transfected HEK293 cells.

Additionally, if the presence of the D297I mutant $\beta$ I-tubulin caused resistance in individual cells, it is reasonable to expect this would enrich the proportion of mutant $\beta I-$ tubulin expressing cells in the population through a proliferative advantage of these cells in drug-treated conditions. Over the complete time course of $144 \mathrm{~h}$ in concentration- 
response studies, there is potential for successfully transfected cells to increase their proportion in the total cells over time. Cells that were less resistant due to less mutant tubulin expression would more readily undergo drug-induced apoptosis.

\section{Rationalising the abundance of tGFP-D297I- $\beta$ I-tubulin expression in HEK293 cells}

The 1A9 cell line was shown to express tGFP- $\beta$ I-tubulins in relatively high abundance, although this expression was not detected by Western blotting or immunocytochemistry at $144 \mathrm{~h}$ following transfection. Loss of transgene expression from episomal DNA both in vitro and in vivo is widely acknowledged. This loss is mediated through nuclease activity or remodelling of chromatin architecture (Hasse, Schulz et al. 1992, Bishop, Ramalho et al. 2006, Riu, Chen et al. 2007) particularly within expression constructs utilising a viral promoter sequence to drive protein expression (Qiu, Leung et al. 2011). Retention of episomal expression over time is highly variable between cell lines due to differences in the activity of plasmid degradation processes, along with differences in the rates of plasmid dilution through cellular proliferation. Rapidly proliferating cell lines such as HEK293 and 1A9 undergo comparatively short mitotic cycles, depleting the plasmid copy number at a greater rate than cell lines with lower proliferative characteristics. This contributes to the earlier loss of episomal expression than that seen in other cell lines with a lower proliferative capacity. A combination of these properties specific to the 1A9 cell line may have led to the high rate of episomal expression loss seen in cultures transfected with our particular tGFP-D297- $\beta$ I-tubulin overexpression construct.

It was surprising that the relative mutant $\beta$ I-tubulin abundance, when measured by Western blot, was lower than expected given that HEK293 cells are widely considered an excellent cell line for overexpression studies. The presence of the tGFP-fusion tag on the carboxylterminus of the $\beta$ I-tubulin monomer was suspected of causing a disruption in epitope recognition by the primary antibody, but assessment with an alternative primary antibody 
specific for an amino-terminus antigen showed no significant difference in the $\beta I$-tubulin expression profile. Western blot data obtained under these experimental conditions may not have produced true representations of the actual tGFP content in these transfected cultures.

Fusion proteins are susceptible to cleavage during storage of protein lysates, even with the addition of appropriate protease inhibitors. As cell samples were stored at $-80{ }^{\circ} \mathrm{C}$ before complete sets were examined by western blotting, potential breakdown of fusion-tagged$\beta$ I-tubulin may have reduced the total tGFP- $\beta$ I-tubulin detected by immunoblotting. Denaturing acrylamide gels were examined for free tGFP fluorescence (i.e. cleavage products of tGFP- $\beta$ I-tubulin) by scanning for GFP fluorescence: however, signal was not detected in the expected $25 \mathrm{kDa}$ region where GFP should migrate. This was likely due to the linearisation of tGFP tertiary protein structure during lysis and separation by SDSPAGE, removing the conformational arrangement required for fluorophore activity. Alternatively, levels of detected $\beta \mathrm{I}$-tubulin may have been reduced during lysate preparation as over expression products have a tendency to saturate protein folding mechanisms, leading to agrosome formation and activation of protein stress responses (García-Mata, Bebök et al. 1999). The presence of low level punctate tGFP expression observed within some cells in confocal imaging (Figure 2.3.10) suggests overexpression of tGFP- $\beta$ I-tubulin in HEK293 cells may generate a low level of insoluble aggregates. Insoluble aggregates would have been removed during clarification of the cell samples and removal of lipid components and other undesired cellular debris. The level of tGFP aggregates may also increase during the lytic process and subsequent preparation for immunoblotting as the presence of detergents and reducing agents pull tGFP- $\beta$ I-tubulin out of a soluble conformation. This is another possible reason that the level of mutant expression in HEK293 cell samples appeared to be lower than expected. 
Endogenous tubulin synthesis is highly responsive to autoregulatory feedback and the introduction of exogenous tubulins by transfection (Gonzalez-Garay and Cabral 1995). The stoichiometric requirement of $\alpha$ - $\beta$-tubulin heterodimer formation means free $\beta \mathrm{I}$ tubulin in the cytosol is rapidly degraded after synthesis if not incorporated into a dimeric configuration. Additionally, fusion constructs display comparatively shorter turnover times than endogenous isoforms, which exhibit greater stability in an unperturbed system. This factors, combined with a potentially enhanced $\beta$ I-tubulin turnover from the disproportionate stoichiometry of overexpression products contribute to the possibility that the low tGFP- $\beta$ I-tubulin signal detected in Western blot analysis may under represent the contribution of exogenous $\beta I$-tubulin in the system.

\section{Functionality of tGFP-tagged- $\beta$ I tubulin in a biological context}

Confocal microscopy of live HEK293 cell cultures allowed for a visual assessment of mutant $\beta$ I-tubulin subunits in their native physiological environment. Before correlating the presence of D297I mutant $\beta$ I-tubulins to any potential shift in concentration-responses observed following treatment with Pel A or Lau, transfected HEK293 cells were examined morphologically. The mutant $\beta$ I-tubulin subunits had the potential to disrupt normal microtubule dynamics; thus, an examination of microtubule polymers with tGFP-tagged $\beta I$-tubulin integrations was important. Polymerised microtubules were observed with both tGFP- $\beta$ I-tubulin and tGFP-D297I- $\beta$ I-tubulin integration into the microtubules, in addition to functional associations with physiological structures such as the centrosome complex and the chromosomes. As normal microtubule morphology was seen, the presence of the $25 \mathrm{kDa}$ tGFP tag did not likely perturb biological function within the system. Mutant $\beta \mathrm{I}-$ tubulin subunits were readily incorporated into microtubule polymers, and were not simply present within the cytosol, lacking appropriate biological activity. In other studies utilising

the same pRCHA $\beta$ I plasmid (Wang, Tian et al. 2006) to introduce a small 9-amino acid hemagglutinin (HA) epitope-tubulin fusion protein noted that the presence of this sequence 
did not alter the biological function of the tubulin subunits, nor processes of dynamic equilibrium.

In conclusion, the HEK293 cell line was validated as a suitable biological system for use in our investigations of the D297 amino acid residue and its predicted molecular interactions with Pel A or Lau. The 1 A9 cell line demonstrated higher levels of mutant $\beta I-$ tubulin expression; however, the transient duration of expression severely limited the scope of this cell line for use in our investigation. A larger shift in potential concentrationresponses would be expected in a system with greater mutant $\beta$ I-tubulin presence; however, the highly consistent nature of results seen in HEK293 cells was considered a more valuable parameter for the assessment of concentration-response shifts resulting from decreased PelA or Lau binding affinity. For this reason, transfection of HEK293 cells was optimised for application in all further concentration-response studies, making use of a small but at least highly consistent introduction of mutant $\beta$ I-tubulin into the cell population. Cells appeared to display no significant alteration in proliferation phenotype in medium containing no microtubule-stabilising compound, and demonstrated tGFP- $\beta$ I-tubulin integration into biologically active microtubule polymers. 


\section{Future directions:}

\section{Improved accuracy in quantification of mutant tubulin}

Western blotting in tGFP- $\beta$ I-tubulin expression studies revealed mutant protein abundance at levels lower than expected for HEK293, a cell line well known for robust expression of plasmid-encoded proteins in over-expression studies. This raised questions regarding the nature of the tGFP- $\beta$ I-tubulin expression quantification by Western blotting and whether it was in fact, representative of exogenous protein in this model. Immunological probing with primary antibodies against epitope recognition sequences at both the carboxy-terminus and amino-terminus determined that the presence of the tGFP fusion-tag on $\beta I$-tubulin did not alter recognition of the target antigen on $\beta$ I-tubulin, thus was an unlikely reason for the low abundance detected for these exogenous proteins. To determine if transfected HEK293 cells were producing more tGFP- $\beta$ I tubulin than the immunoblotting suggested, a quantitative approach by mass-spectrometry could be utilised in future investigations. Peptide mass fingerprint analysis by matrix-assisted laser desorption ionisation-time of flight mass spectrometry (MALDI-TOF) represents a powerful technology capable of identifying and quantifying the presence of specific protein species within a sample. Because the mutant $\beta$ I-tubulin constructs used in this investigation were constitutively expressed with a $25 \mathrm{kDa}$ tGFP fusion tag, peptide fingerprint analysis by MALDI-TOF following protein separation by high-performance liquid chromatography (HPLC) is possible due to the presence of the unique tGFP peptide sequence on the mutant $\beta$ I-tubulin sequence, but not the endogenous $\beta$ I tubulin contribution. Traditionally, mass spectrometry based methodologies utilising isotope-coded affinity tags (ICAT) have been able to deduce the relative abundance of specific proteins in complex mixtures, as was done in a microsomal analysis study (Han, Eng et al. 2001). Similarly to ICAT-derived analysis, the tGFP-fusion tag on our protein of interest may be utilised in the identification of unique 
peptide signatures. Recent advances in LC-MALDI-TOF make peptide analysis of fully sequenced genomes (Blackstock and Weir 1999) possible, with complementary validation by other proteomic methods offering support for the use of MALDI-TOF in large scale quantitative analysis (Hessling, Buttner et al. 2013).

In the present investigation, we were interested in the relative abundance of just two specific protein signatures. By making use of the unique peptide fragmentation signatures of $\beta$ I-tubulin, a workflow for comparing the relative frequency of endogenous $\beta$ I-tubulin to tGFP- $\beta$ I-tubulin within individual chromatographic fractions is possible. Endogenous $\beta I-$ tubulin signature events within lighter protein fractions (as defined by molecular weight) may be compared directly to equivalent peptide fragmentation signatures detected within heavier fractions that correspond to the tGFP-tagged $\beta I$-tubulin isotype. Peptide fragmentation signatures for tGFP itself would also be represented within heaver fractions, allowing a secondary comparison of $\beta \mathrm{I}$-tubulin/tGFP event frequency. The relative frequency of each detection correlates directly to the abundance of each $\beta I$-tubulin isotype within a sample. Using this approach in future experiments would offer an enhanced level of clarity surrounding the nature of plasmid encoded expression, supporting the low abundance of tGFP-D297I- $\beta$ I-tubulin observed or highlighting possible artefacts within the immunoblotting approach used in this investigation to estimate the ratio of mutant $\beta 1$ tubulin to endogenous $\beta 1$-tubulin.

In addition, the detection of free tGFP peptide signatures $(25 \mathrm{kDa})$ within very light HPLC fractions would provide some insight into the potential role of tGFP-fusion cleavage from plasmid encoded $\beta$ I-tubulins which may have contributed to the reduced mutant- $\beta$ I tubulin detected by immunoblotting. Once the GFP moiety is cleaved off the mutant tubulin in the 
cell, the mutant protein would electrophorese with the same mobility as the endogenous tubulin and would thus be falsely recognised as endogenous and not transfected $\beta 1$-tubulin, giving an artificially low Western blot estimation of transfected protein relative to endogenous.

\section{Generation of a stable transfected cell line}

To further examine the characteristics of mutant $\beta$ I-tubulin binding interactions with Pel A or Lau, the generation of HEK293 cell lines with a stable genomic integration of plasmidencoded mutant $\beta$ I-tubulin sequences would be highly advantageous. A cell line with this genetic property would likely improve the resolution of any concentration-response investigations due to expression of mutant $\beta$ I-tubulin throughout the clonal population. Likewise, a stable integration of the mutant sequence into additional cell lines such as PTXresistant 1 A9 cells would allow further characterisation of drug interactions in a more clinically relevant model, while avoiding complications surrounding loss of mutant $\beta \mathrm{I}-$ tubulin expression in the culture over time. 


\section{Chapter 3. Investigations into the differential drug sensitivity of mutant tGFP-D297I- $\beta I-t u b u l i n$ in HEK293 cells}

\subsection{Introduction}

The material presented within chapter three of this thesis details the quantifiable changes in properties of a cell population after transfection with the mutant $\beta I$-tubulin species. These assessments were carried out through concentration-response studies and examination of alterations in D297I mutant tubulin polymerisation states in the presence of Pel A, Lau, PTX and IXA.

\subsubsection{Introduction to cytotoxicity screening and cell proliferation assays}

Following the introduction of high-throughput screening methodologies, the number of chemical compounds of both synthetic and natural origin that have been shown to exhibit bioactive properties is quickly expanding. At present, there are 977,499 individual compounds entered in the NCBI Database PubChem Integrated Platform of Small Molecules and Biological Activities database (Bolton, Wang et al. 2008, 2014) with bioactivity assessments carried out through one or more assay formats. Compounds are usually assessed across multiple assay formats, each designed to identify a specific type of bioactive property. Compounds demonstrating cytotoxic properties are of particular interest for drug development in that these represent potential candidate molecules for the development of new therapeutics. Two key bioassay types used in the identification and assessment of anti-cancer biomolecules are those designed to measure cytotoxicity or antiproliferative characteristics in vitro and provide further characterisation of a compound following identification. Compounds Pel A, Lau, PTX and IXA are all thoroughly characterised as potent cytotoxic agents with concentration-dependent properties in vitro. 
In order to characterise the specific activities of these compounds against cells with a mutant protein-target, this study has made extensive use of the reductive properties of the 3-(4,5-Dimethyl-2-thiazolyl)-2,5-diphenyl-2H-tetrazolium salt (MTT). The MTT cell proliferation assay initially described by Mosmann (1983) is a robust, widely applied method for the examination of compound-induced growth inhibition in cells, particularly in cells with a high correlation between rates of metabolic process and proliferative capability. MTT is reduced in the cytosol of cells with functional NAD(P)H-dependent oxidoreductase enzymes, although a degree of reductive activity occurs with additional cytosolic and membrane bound elements (Berridge, Herst et al. 2005). This reductive capacity may then be correlated to metabolic activity by measuring the optical absorbance of the purple formazan product at $570 \mathrm{~nm}$. The intensity of absorbance is a useful indicator for cell viability in culture under controlled conditions and thus allows determination of the concentration-response of these cells in the presence of compounds of interest.

\subsubsection{Introduction to in situ tubulin dynamics}

As detailed throughout chapter 1 , the cytoskeleton is a highly dynamic system undergoing constant rearrangements to facilitate necessary cellular processes. Microtubules exhibit dynamic polymerisation behaviours, particularly during the major cellular rearrangements leading up to and during mitosis. Methods designed to examine the in situ state of tubulin polymerisation allow separation of insoluble (polymerised) tubulin fractions from soluble (non-polymerised) fractions as an indicator of microtubule polymer activity within a cell population. Following treatment with a MTA, the effect of a compound on the dynamic equilibrium of tubulin can be examined through observing a shift of tubulin subunits between states of solubility. MTAs promote the stabilisation of tubulin heterodimers into polymerised microtubules, shifting the dynamic equilibrium of these proteins towards favoured presentation in the insoluble tubulin fraction. Giannakakou, Sackett et al. (1997) 
utilised fractionation of tubulin by solubility state to demonstrate cells with PTX resistance showed impaired polymerisation responses in the presence of PTX. In the current study this approach is utilised to investigate the solubility state of WT HEK293 cell cultures compared to tGFP-D297I- $\beta$ I transfected HEK293 cells when treated with Pel A, to determine whether the presence of this mutation causes HEK293 cells causes inhibition of Pel A induced polymerisation-responses. 


\subsubsection{Aims of this Chapter}

A) Evaluate the concentration-response of cells with an expressed D297 point mutant $\beta I$-tubulin population in response to Pel A and Lau

The primary aim of this chapter is to evaluate the biological effect of introduced tGFPD297I- $\beta$ I-tubulin mutant subunits on the concentration-response of transfected cell populations treated with Pel A or Lau. It was hypothesised that the presence of the D297 substitution may alter the proliferative response of cells to Pel A and Lau as the result of a disruption in normal ligand association within mutant subunits. As a consequence, transfected cells will display sensitivity or resistance phenotypes as a result of the presence of point mutant $\beta$ I-tubulin subunits and the magnitude of these changes may differ between treatment with Pel A or Lau. This chapter seeks to evaluate the nature of these potential responses.

B) Examine the concentration-response of transfected cells treated with PTX or IXA to determine the effect of the point mutations presence, independent of direct ligand binding to the D297I substitution.

A second aim of this chapter is to determine if the presence of the tGFP-fusion tag or point mutation in a portion of the total $\beta I$-tubulin in the cell population affects the concentrationresponse of these transfected cells to MSAs that do not associate directly to $\beta I$-tubulin in the region of these mutations. This will generate insights into the effects of the mutation on global conformation within the tubulin monomer, which has the potential to impact concentration-response. This chapter aims to determine if mutant $\beta$ I-tubulin subunit presence alters proliferation in the presence of PTX and IXA, so any changes seen in Pel A and Lau treatments are likely the consequence of disrupted ligand interactions at amino acid residue 297. 


\section{C) Examine microtubule polymerisation states for D297I- $\beta$ I-tubulin point mutation transfected cells in response to Pel A}

The presence of $\beta$ I-tubulin subunits containing the D297I amino acid substitution may display alterations in dynamic behaviours in response to drug treatment by Pel A compared to WT cultures. It was hypothesised that the presence of D297I in a portion of the total $\beta \mathrm{I}-$ tubulin may allow a level of dynamic instability in microtubule polymerisation behaviours to be maintained in the presence of drug. This chapter aims to investigate these potential alterations and if found, rationalise the contribution of this effect in relation to any changes seen in the concentration-response of transfected cells treated with Pel A. 


\subsection{Materials and Methods}

\subsubsection{Cellular proliferation of D297I mutant $\beta 1$-tubulin HEK293 by MTT in response to microtubule-stabilisers}

To assess the anti-proliferative activity of microtubule-stabilising compounds on HEK293 cells, a standard approach was used to find the concentration of Pel A, Lau, IXA or PTX at which $50 \%$ of proliferative activity was inhibited $\left(\mathrm{IC}_{50}\right)$ as determined by a half $\log$ concentration-response treatment. HEK293 cells transfected with D297I mutant tGFP- $\beta 1$ tubulin or wild type tGFP- $\beta 1$-tubulin and mock-transfected (treated with Lipofectamine $3000^{\circledR}$ only) were seeded in 96-well tissue culture plates (Microtest ${ }^{\mathrm{TM}}$ Tissue culture plate, Falcon, Becton Dickson) at $1 \times 10^{4}$ cells / well in $100 \mu \mathrm{L}$ complete growth medium and allowed to adhere overnight. Microtubule-stabilising agents were added after a minimum of $48 \mathrm{~h}$ post-transfection to ensure consistency in levels of exogenous gene expression at the time of culture treatment. Cells were treated the following day with the various microtubule-stabilising agents by addition of $400 \mathrm{nM}$ compound to the first well in duplicate followed by a half-log concentration serial dilution down the 96-well plate. Media transfers were carried out as gently as possible so as not to detach any cells. The plates were then incubated for $72 \mathrm{~h}$ at $37^{\circ} \mathrm{C}$ before addition of $20 \mu \mathrm{L}$ MTT $(5 \mathrm{mg} / \mathrm{mL}$ in sterile-filtered PBS). Plates were incubated for $2 \mathrm{~h}$ at $37^{\circ} \mathrm{C}$ to allow the development of purple formazan products. Crystalline formazan precipitates were solubilised with the addition of $100 \mu \mathrm{L}$ per well solubilising solution (10\% SDS, 45\% DMF by volume in Milli-Q water $\mathrm{pH}$ adjusted by acetic acid to 4.50). After incubation at $37^{\circ} \mathrm{C}$ overnight, infrared absorbance at $570 \mathrm{~nm}$ was determined spectrophotometrically using a multiwell-plate reader (Enspire 2300, Perkin Elmer). Using growth medium-only well absorption as a background, untreated control wells served as a baseline for normal cellular proliferation. Cellular proliferation of drug-treated samples was calculated via subtraction of the background absorbance and expressed as a percentage of the control absorbance adjusted for 
background absorbance (sample absorbance - background absorbance / untreated absorbance - background absorbance) $\mathrm{x} 100$. These proliferation data were utilised to generate half maximal inhibitory concentration models using Sigma Plot v.11 (Systat Software Inc., Point Richmond, CA, USA) by fitting four parametric logistic curves to a log scale of each stabilising agent concentration against the corresponding cellular proliferation as a percentage of control. 
Sigma Plot v.11 utilises the following equation in the generation of these models:

$$
\text { Cellular Proliferation (\% Control) }=\frac{\text { Max }- \text { Min }}{1+\left(\frac{\text { concentration }}{I C_{50}}\right)^{\text {Hillslope }}}
$$

Max indicates the maximum measurement and Min the minimal value measured.

Each of the four compounds, Pel A, Lau, PTX and IXA, were assessed against each of the three HEK293 variants (mock-transfected, tGFP-tubulin transfected and the tGFP-D297Itubulin mutant transfected) to generate at least seven biological replicates for each cell variant/drug treatment combination with each carried out with at least two technical replicates. Prior investigations with three to four biological replicates indicated this study would benefit from a larger pool of biological replicates to improve on significance of any observed changes in concentration-response.

\subsubsection{In situ polymerisation of $\beta 1$-tubulin containing D297I mutant isotype subunits in response to peloruside $A$}

Cells were transfected in 6-well tissue culture plates as described in section 2.2 .5 with D297I mutant tGFP- $\beta 1$-tubulin and allowed to grow to confluence before the growth medium was aspirated and replaced with $1.5 \mathrm{~mL}$ complete growth medium supplemented with Pel A at concentrations of 0,40 or $100 \mathrm{nM}$ for $16 \mathrm{~h}$. Cells were briefly rinsed twice with $1 \times$ PBS before lysis by incubation at $37^{\circ} \mathrm{C}$ in the dark with $400 \mu \mathrm{L}$ hypotonic buffer (1 $\mathrm{mM} \mathrm{MgCl} 2,2 \mathrm{mM}$ EGTA, $1 \%$ Noniodet P-40 by volume, $50 \mathrm{mM}$ Tris-HCL (pH to 6.8) and $10 \mu \mathrm{L} / \mathrm{mL}$ protease inhibitor cocktail I (Sigma-Aldrich, Auckland, NZ) for $5 \mathrm{~min}$. Following lysis, polymerised and soluble tubulin fractions were separated by centrifugation at $14,400 \mathrm{~g}$ for $10 \mathrm{~min}$ at room temperature in $1.5 \mathrm{~mL}$ Eppendorf tubes. The soluble protein content was collected by the transfer of the supernatant to new $1.5 \mathrm{~mL}$ Eppendorf tubes and the insoluble fraction resuspended in $400 \mu \mathrm{L}$ hypotonic buffer. All samples were stored on 
ice during subsequent steps. Samples were prepared for electrophoretic separation by SDSPAGE by diluting $16 \mu \mathrm{L}$ of protein sample with $5 \mathrm{x}$ Laemmli reducing buffer containing $10 \% \beta$-mercaptoethanol before complete denaturation by boiling at $95^{\circ} \mathrm{C}$ for $5 \mathrm{~min}$. Twenty $\mu \mathrm{L}$ of each sample was run on a $1.5 \mathrm{~mm}, 10 \%$ polyacrylamide resolving gel with a $6 \%$ polyacrylamide stacking gel alongside $1.5 \mu \mathrm{L}$ of molecular weight ladder (Dual Colour Precision Plus ${ }^{\mathrm{TM}}$, Bio-Rad Laboratories). Electrophoretic separation of sample protein's was carried out at $120 \mathrm{~V}$ for $1.5 \mathrm{~h}$ or until the dye front had completely migrated through the resolving gel. Proteins were transferred by electrophoresis for $1 \mathrm{~h} 20 \mathrm{~min}$ at a constant $300 \mathrm{~mA}$ to a methanol-activated low fluorescence background PVDF membrane (Immobilon-P, Millipore Corp, MA, USA). Following protein transfer, membranes were blocked overnight at $4^{\circ} \mathrm{C}$ on a rocking platform using 5\% BSA in PBST. Acrylamide gels containing non-transferred proteins fixed for $1 \mathrm{~h}$ at room temperature in $50 \% \mathrm{EtOH}$ and $3 \%$ ortho-phosphoric acid diluted in Milli-Q water. Gels were then washed three times with Milli-Q water before submersion in Coomassie staining solution $\left(0.06 \%\right.$ Coomassie ${ }^{\circledR}$ Brilliant blue G-250 (Biorad Laboratories, Hercules, CA, USA) dissolved in 34\% methanol, $17 \%$ ammonium sulphate and 3\% phosphoric acid by volume) and left to develop on a rocking platform at room temperature for two days before being examined for transfer quality. Conditions for immunological probing of blocked membranes along with individual experimental control parameters are described in detail with the corresponding results sections. All membranes were washed two times between antibody incubations with PBST for $10 \mathrm{~min}$ at room temperature followed by a third wash with $1 \mathrm{x}$ PBS for $10 \mathrm{~min}$ at room temperature. Probed membranes were scanned and analysed as described in section 2.2.7. 


\subsubsection{Secondary assessment of D297I mutant $\beta 1$-tubulin presence in HEK293 cells}

Immunological detection methods described in section 2.2.7, while able to detect the presence of tGFP-tubulins, resulted in a surprisingly low level of tGFP- $\beta 1$-tubulin detected and may misrepresent the actual tGFP-D297I content within the cells utilised in proliferation assays. As a secondary assessment, tGFP-D297I-tubulin mutant lysates were analysed further by western blotting as described in section 2.2.7 with a focus placed on the detection of the tGFP fusion sequence rather than $\beta 1$-tubulin itself. tGFP containing samples were transferred to low background florescence (Immobilon-P, Millipore Corp, MA, USA) after electrophoretic separation by SDS-PAGE as described previously. Immunological probing was carried out using a 1:1000 dilution of mouse anti-tGFP monoclonal antibody (TA140041, Origene Technologies, Rockville, MD, USA) in 1\% BSA-TBS for $2 \mathrm{~h}$ at room temperature followed by the secondary goat anti-mouse AlexaFluor® 488 (A-11001, Life Technologies) in 1\% BSA-TBS for $1.5 \mathrm{~h}$ at room temperature. After each antibody incubation, membranes were washed twice in PBST for $10 \mathrm{~min}$ at room temperature, followed by a third $10 \mathrm{~min}$ wash in TBS at room temperature. The presence of tGFP- $\beta 1$-tubulin in samples was scanned and analysed as described in section 2.2.7.

\subsection{Results}

\subsubsection{Assessment of transfection impact on HEK293 in response to PTX and IXA}

HEK293 cells transfected with tGFP- $\beta$ I-tubulin, tGFP-D297I- $\beta$ I-tubulin or mock transfected cells were tested with PTX and IXA using the MTT cell proliferation assay as described in 3.2.1. This allowed for changes in drug sensitivity to be assessed in the D297I point mutation transfected populations. The D297 amino acid shares no predicted molecular interaction with either PTX or IXA, allowing these drugs to be used as negative controls for the assessment of any alterations to cell susceptibility to Pel A or Lau, attributed 
directly to the presence of mutant- $\beta$ I-tubulin. The D297I point mutation was designed to introduce a small, innocuous change to the $\beta$ I-tubulin amino acid sequence relative to the action of a taxoid site-binding drug. However, even this type of modification could result in changes to the tertiary protein structure. Since any change of this nature has the potential to interfere with a proteins normal biological function, treatment with PTX and IXA offers an opportunity to test whether the altered $\beta$ I-tubulin subunit can shift the concentrationresponse profile of transfected HEK293 cells where no change is expected in comparison to wild type HEK293 cells. No significant shift in $\mathrm{IC}_{50}$ values with either PTX or IXA was observed between either transfection group compared to the wild type mock-transfection control ( $\mathrm{p}>0.05$, Student's t-test). This suggested the D297I point mutation does not contribute any significant disruption to PTX or IXA drug association with $\beta$ I tubulin, nor does the mutation significantly alter the proliferation characteristics of HEK293 cells following transfection. The following figures depict concentration-response curves representative of a single experiment for mock-transfection control, tGFP- $\beta$ I tubulin and tGFP-D297I- $\beta$ I-tubulin transfected cells along with summary data from a minimum of 7 biological repeats. $\mathrm{IC}_{50}$ values were calculated from four- parameter logistic curves fitted to concentration-response data using Sigma Plot v.11.0. 
A)

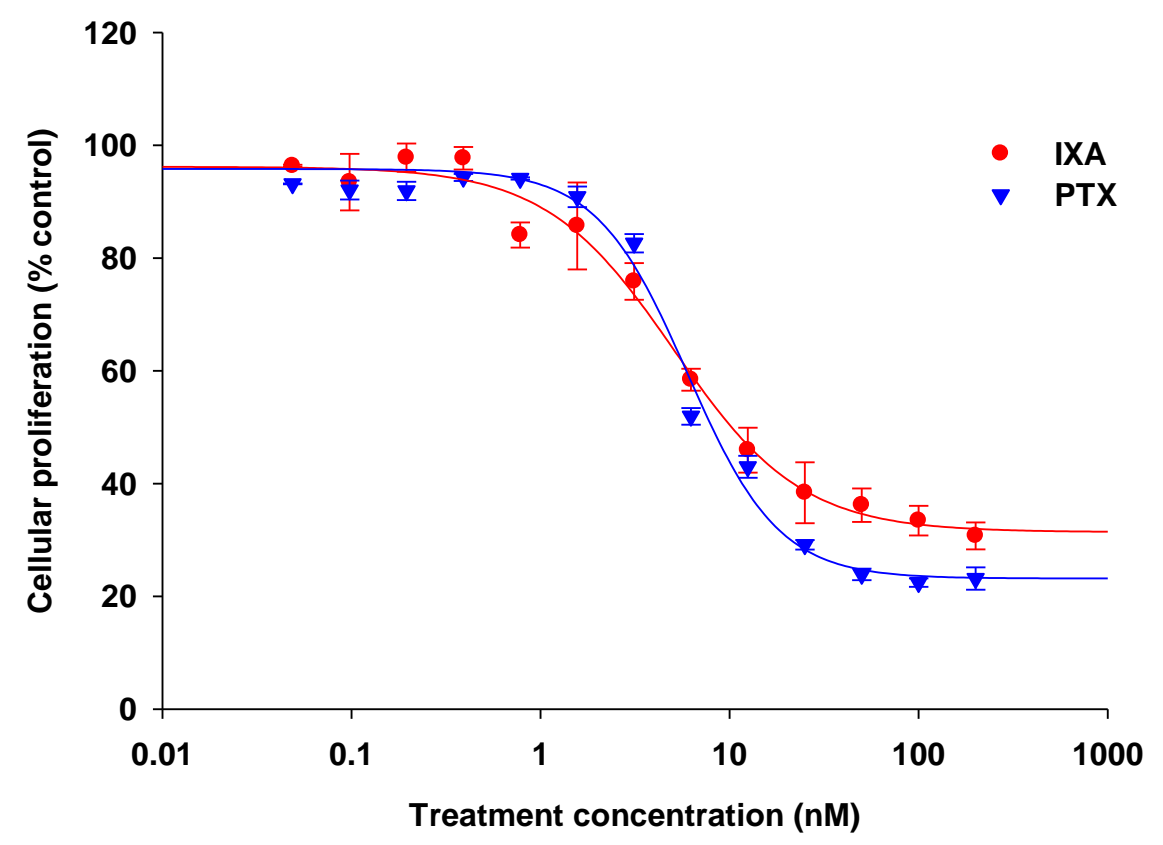

B) IC I $_{50}$ of IXA and PTX on mock-transfected HEK293

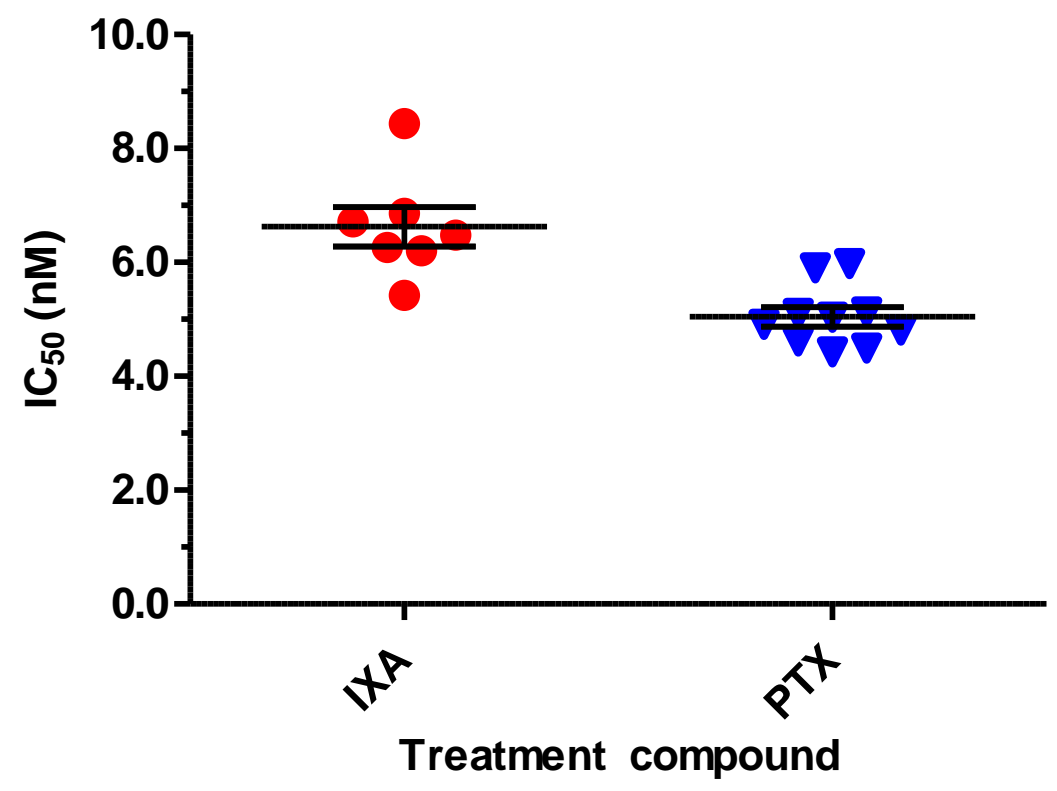

Figure 3.4.1 Concentration-response for mock control transfected HEK293 treated with PTX and IXA.

A) Representative concentration-response curves of mock-transfected (lipofectamine only control) HEK293 cells with IXA and PTX treatment. Mock-transfected HEK293 cells were treated for $72 \mathrm{~h}$ with different concentrations $(\leq 200 \mathrm{nM}$ of IXA or PTX. Cell proliferation was assessed using the MTT assay as described in the methods.

B) IC $C_{50}$ concentration (nM) of IXA and PTX in mock-transfected (lipofectamine only control) HEK293 cells. The $\mathrm{IC}_{50}$ for mock-transfected HEK293 cells were determined by the MTT assay as described in 3.2.1. $\mathrm{IC}_{50}$ data are presented as the mean concentration $(\mathrm{nM}) \pm \mathrm{SEM}(\mathrm{n}=7$ IXA, 10 PTX biological replicates). 
A)

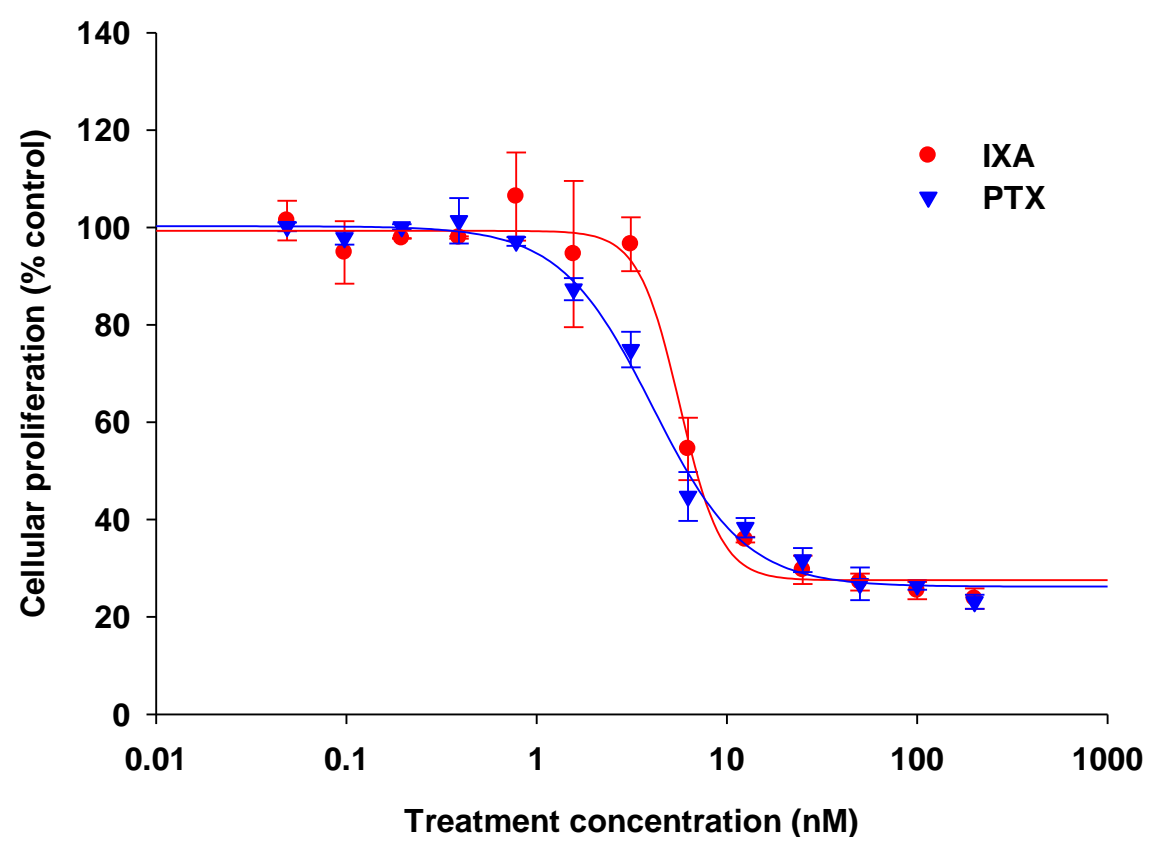

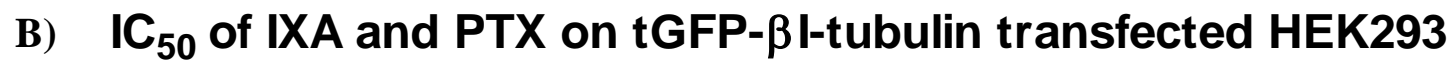

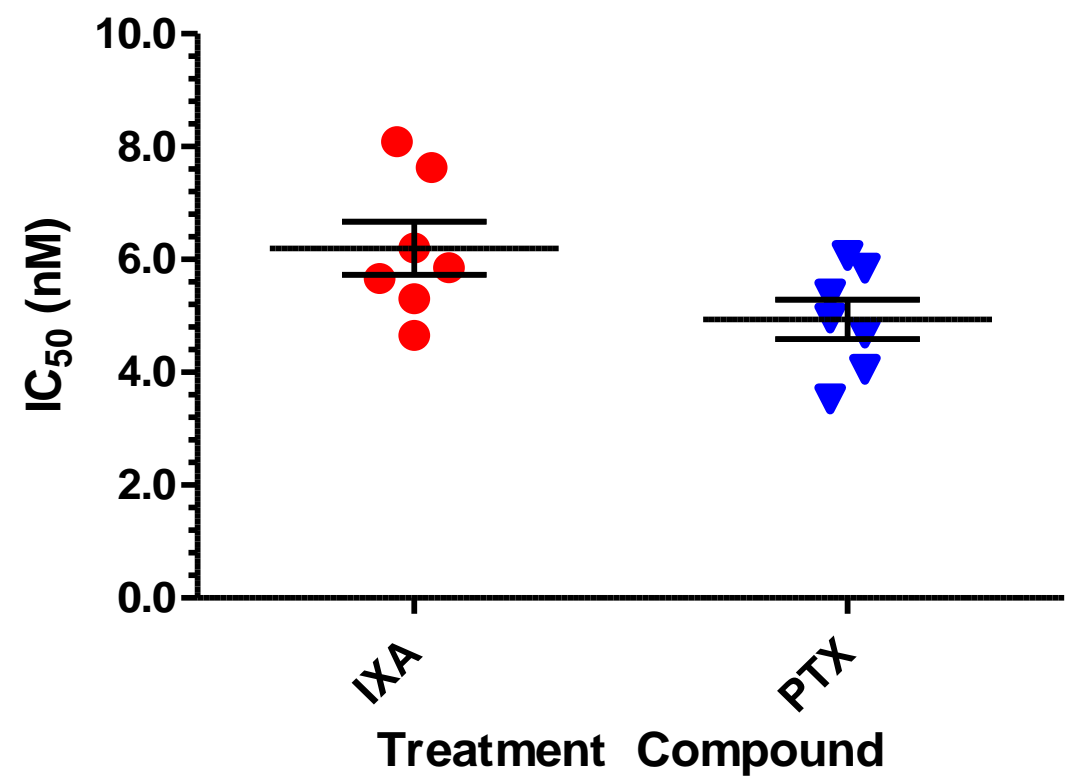

Figure 3.4.2 Concentration-response for tGFP-BI transfected HEK293 cells treated with PTX and IXA

A) Representative concentration-response curves of WT tGFP- $\beta I$-tubulin transfected HEK293 cells with IXA and PTX treatment. WT tGFP- $\beta$ I-tubulin transfected HEK293 cells were treated for $72 \mathrm{~h}$ with different concentrations $(\leq 200 \mathrm{nM})$ of IXA or PTX. Cell proliferation was assessed using the MTT assay as described in the methods.

B) IC $C_{50}$ concentration ( $\left.n M\right)$ of IXA and PTX in WT tGFP- $\beta$ I-tubulin transfected HEK293. The IC 50 for WT tGFP-BI-tubulin transfected HEK293 was determined by the MTT assay as described in 3.2.1. $\mathrm{IC}_{50}$ data are presented as the mean concentration $(\mathrm{nM}) \pm \mathrm{SEM}(\mathrm{n}=7$ biological replicates). 


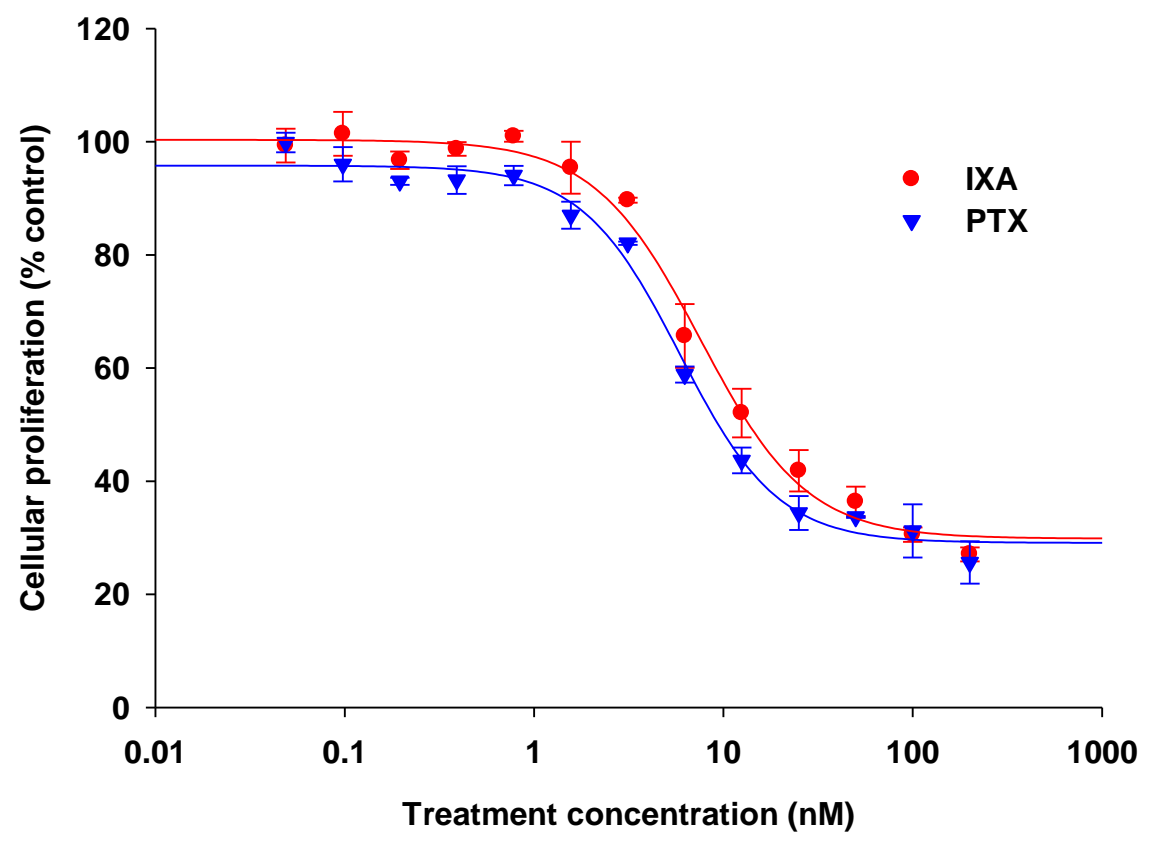

B) IC $50_{0}$ of IXA and PTX on tGFP-D297I $\beta$ I-tubulin transfected HEK293

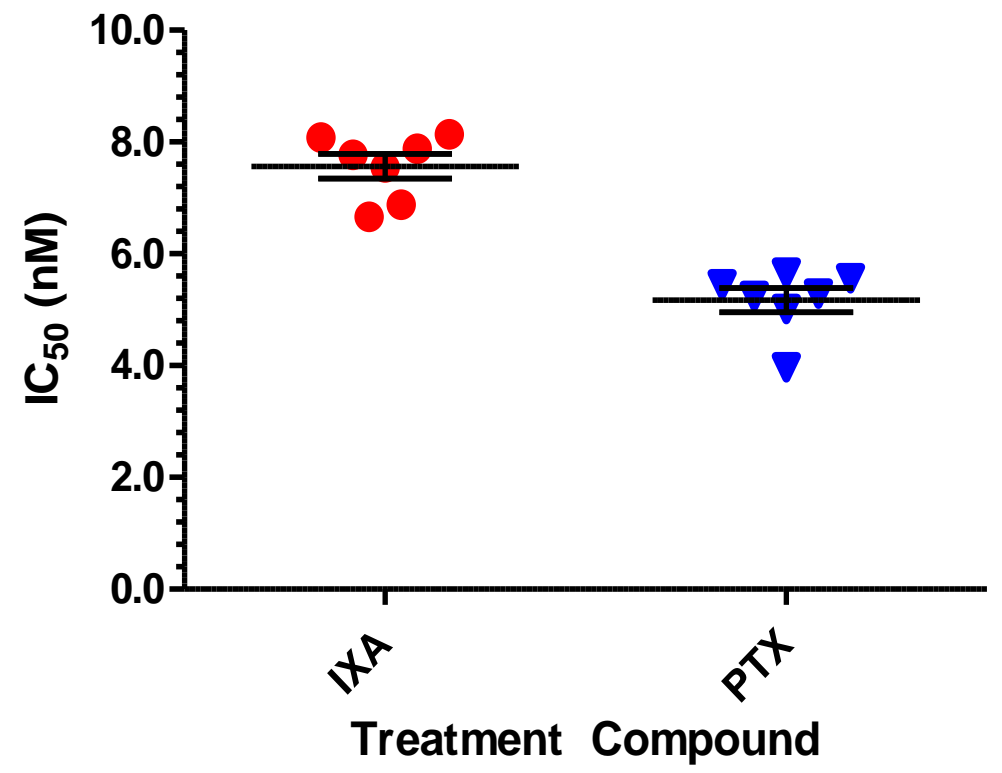

Figure 3.4.3 Concentration-response for tGFP-D297- $\beta$ I mutant transfected HEK293 cells treated with PTX and IXA

A) Representative concentration-response curves of tGFP-D297I- $\beta I$-tubulin transfected HEK293 cells with IXA and PTX treatment. tGFP-D297- $\beta$ I-tubulin transfected HEK293 cells were treated for $72 \mathrm{~h}$ with different concentrations $(\leq 200 \mathrm{nM})$ of IXA or PTX. Cell proliferation was assessed using the MTT assay as described in the methods.

B) IC $C_{50}$ concentration (nM) of IXA and PTX in tGFP-D297I- $\beta$ I-tubulin transfected HEK293. The IC $C_{50}$ for $t$ GFP-D297I- $\beta I$ transfected HEK293 was determined by the MTT assay as described in 3.2.1. $\mathrm{IC}_{50}$ data are presented as the mean concentration $(\mathrm{nM}) \pm \operatorname{SEM}(\mathrm{n}=7$ biological replicates). 


\section{IC $_{50}$ of PTX treated HEK293 transfectants}

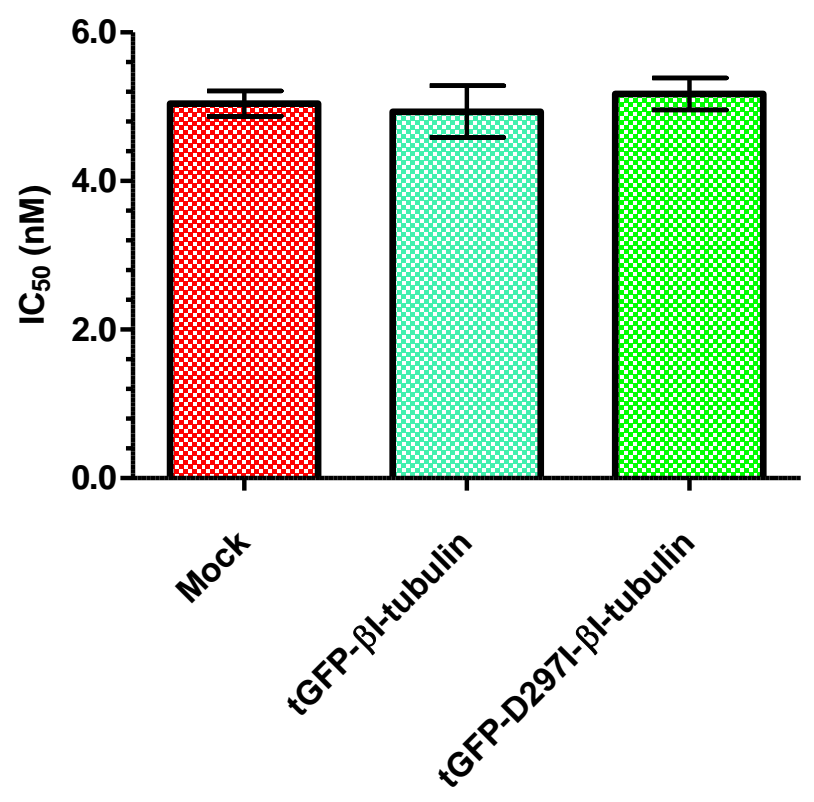

Transfection vector

\section{IC $_{50}$ of IXA treated HEK293 transfectants}

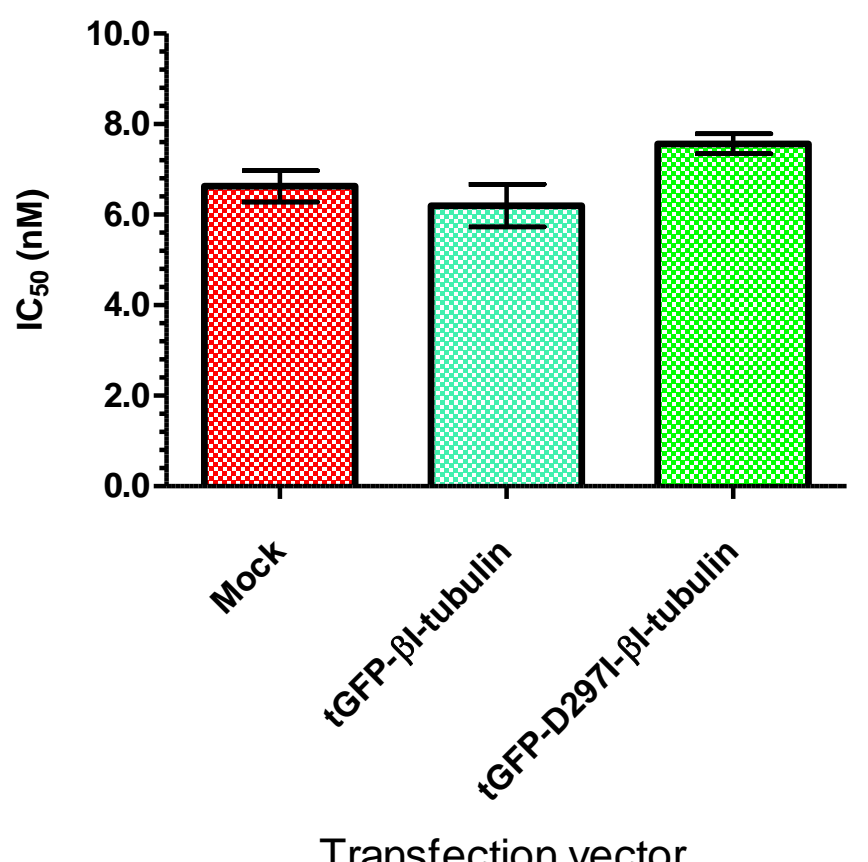

Figure 3.4.4 Summary of IC $C_{50}$ data for PTX or IXA concentration-response treatments in transfected HEK293 compared to mock transfected cells. No statistical significance between transfection vector groups was seen for either PTX or IXA treatment, indicating that the presence of mutant $\beta$ I-tubulin does not significantly alter cellular proliferation in response to PTX or IXA (unpaired Student's t-test $\mathrm{p}>.05)$. $\mathrm{IC}_{50}$ data are presented as mean concentration $(\mathrm{nM}) \pm \mathrm{SEM}(\mathrm{n}=$ 7 biological replicates). 


\begin{tabular}{|c|c|c|c|}
\hline Drug & $\begin{array}{l}\text { IC50 }(\mathbf{n M}) \\
\text { Mock-control } \\
(\mathrm{n}=7)\end{array}$ & $\begin{array}{l}\text { tGFP- } \boldsymbol{\beta} \text { I-tubulin } \\
(\mathrm{n}=7)\end{array}$ & $\begin{array}{l}\text { tGFP-D297I- } \boldsymbol{\beta I} \text { - } \\
\text { tubulin }(\mathrm{n}=7)\end{array}$ \\
\hline IXA & $6.2 \pm 0.3$ & $6.2 \pm 0.5$ & $7.6 \pm 0.2$ \\
\hline PTX & $5.0 \pm 0.2$ & $5.0 \pm 0.3$ & $5.2 \pm 0.2$ \\
\hline
\end{tabular}

Table 3.4.1 Summary IC $C_{50}$ data for IXA or PTX treated HEK293 cells. $\mathrm{IC}_{50}$ were determined by the MTT assay. Data are presented as the mean \pm SEM. No significant difference was found between the three groups with either drug as assessed by unpaired Student's t-test $(\mathrm{P}=>.05)(\mathrm{n}=7$ biological repeats).

\subsubsection{Assessment of transfection impact on HEK293 in response to Pel A or Lau}

As expected, the presence of the D297I mutation within the population had no effect on the sensitivity of the cells to the taxoid site microtubule-stabilising drugs IXA and PTX. This was expected as the introduced mutation is situated well outside the taxoid binding site on $\beta$-tubulin. This suggests that any change observed in the $\mathrm{IC}_{50}$ concentration for Pel A or Lau treatment in D297I point mutation transfected cultures is likely the result of an altered drugtubulin interactions. The following figures depict concentration-response curves for treatment with both Pel A and Lau, representative of a single experiment for mocktransfected control, tGFP- $\beta$ I tubulin and tGFP-D297I- $\beta$ I-tubulin transfected cells along with summary data from a minimum of 7 biological repeats. $\mathrm{IC}_{50}$ values were calculated from four- parameter logistic curves fitted to concentration-response data using Sigma Plot v.11.0. 
A)

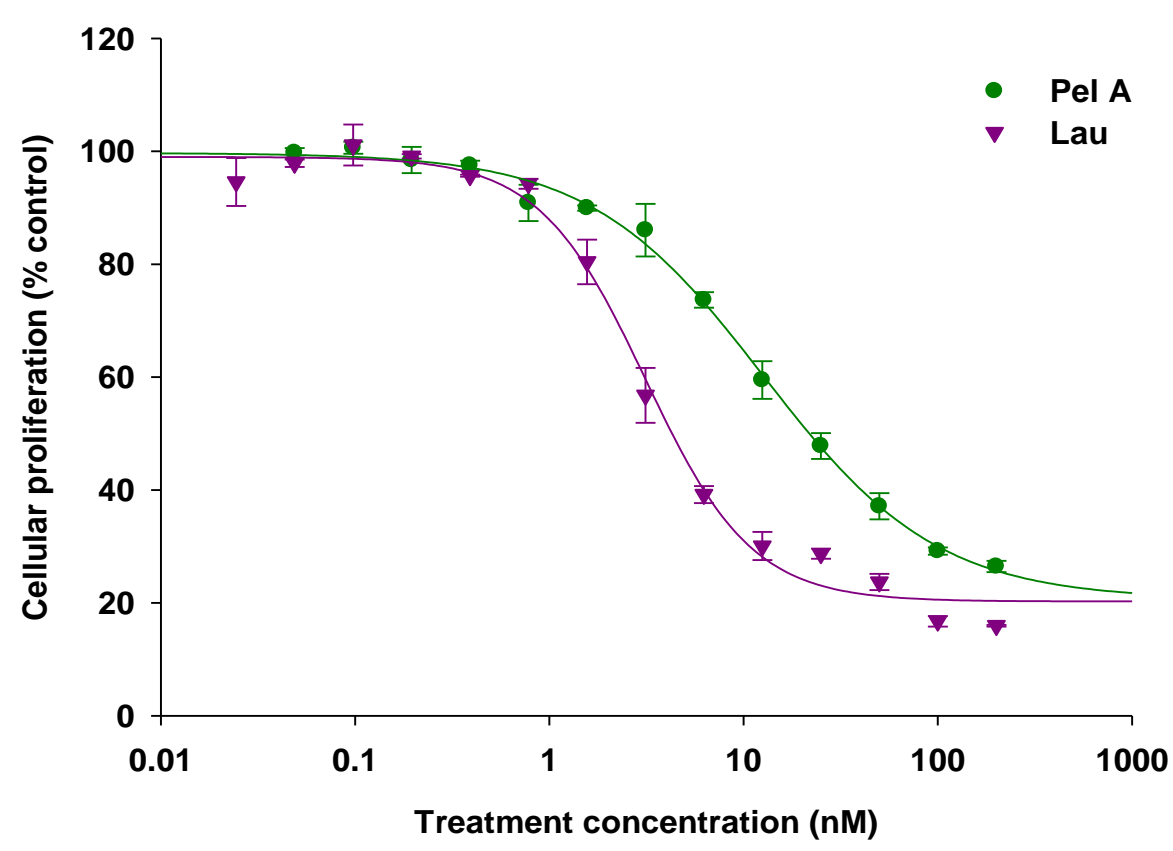

B)

IC $_{50}$ of Pel A and Lau on mock-transfected HEK293

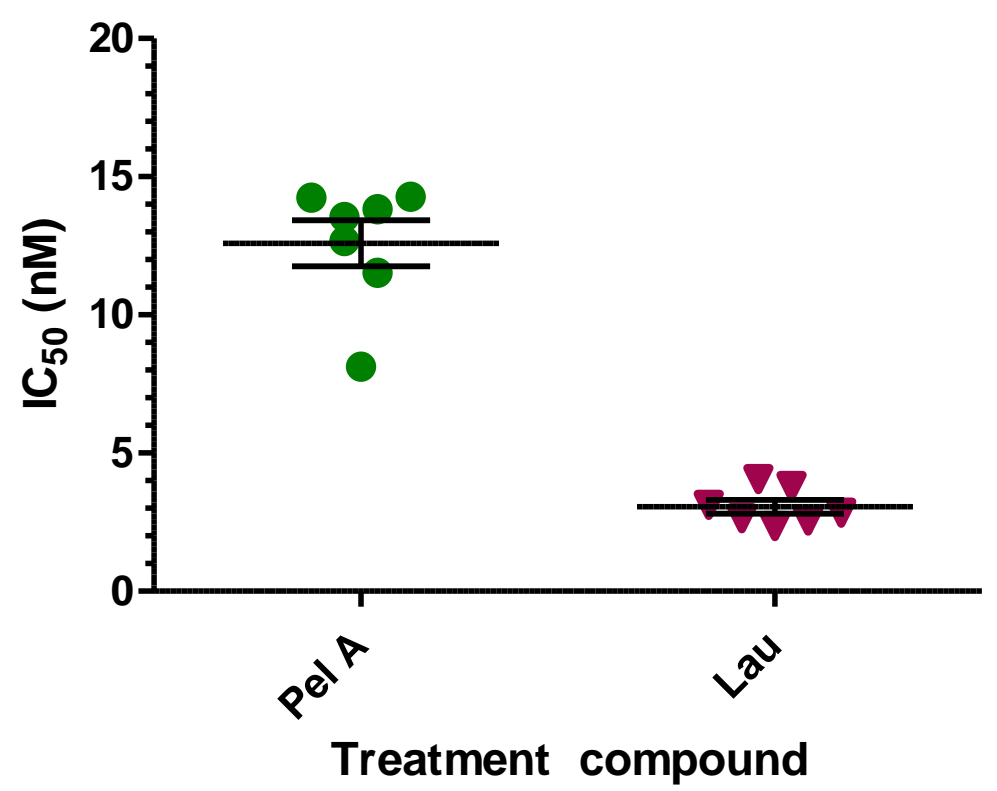

Figure 3.4.5 Concentration-response for mock-transfected HEK293 cells treated with Pel A and Lau.

A) Representative concentration-response curves of mock-transfected HEK293 cells treated with Pel A and Lau. Transfected cells were treated with Pel A or Lau for 72 before measurement of cell proliferation using the MTT assay as described in the methods. B) $\mathrm{IC}_{50}$ of Pel A and Lau in mocktransfected HEK293 cells. The IC 50 concentrations (nM) for mock-transfected HEK293 were determined by the MTT assay. IC $C_{50}$ data are presented as the mean $I C_{50}(n M) \pm S E M(n=7$ biological replicates). 
A)

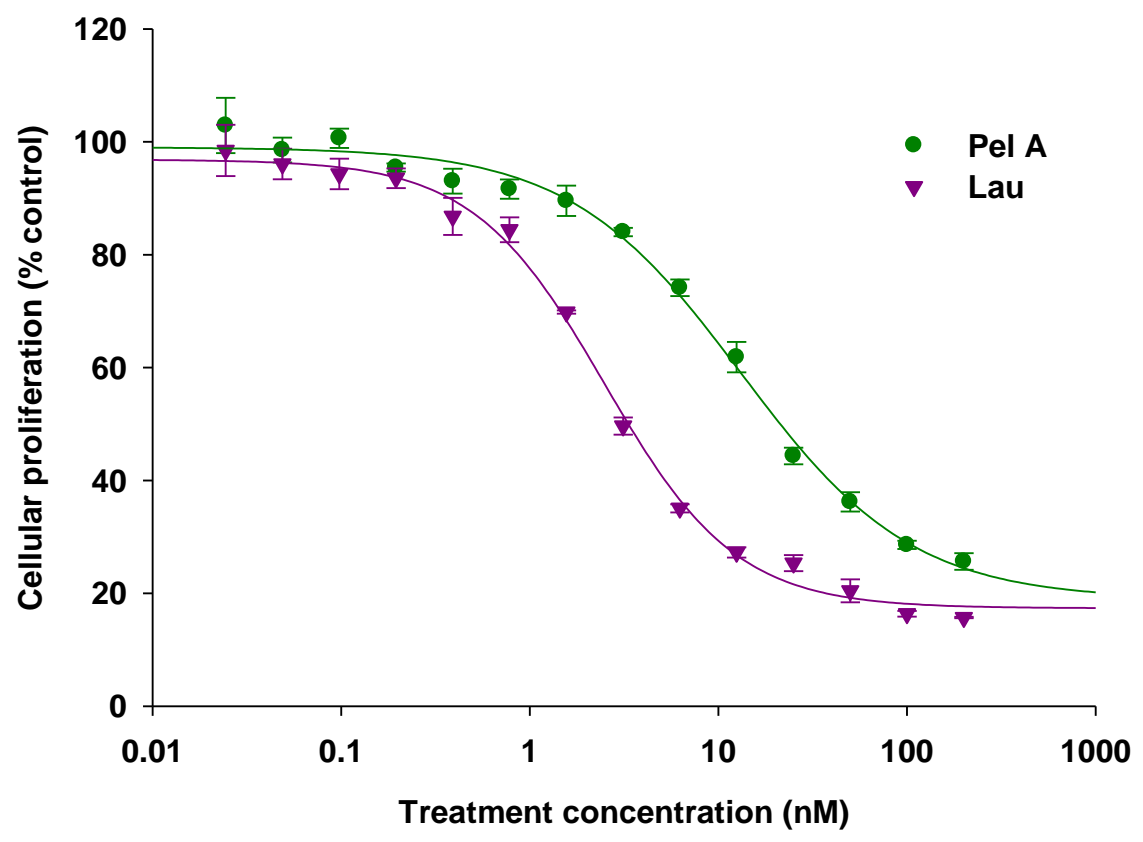

B) $\mathrm{IC}_{50}$ of Pel A and Lau on tGFP- $\beta$ I-tubulin transfected HEK293

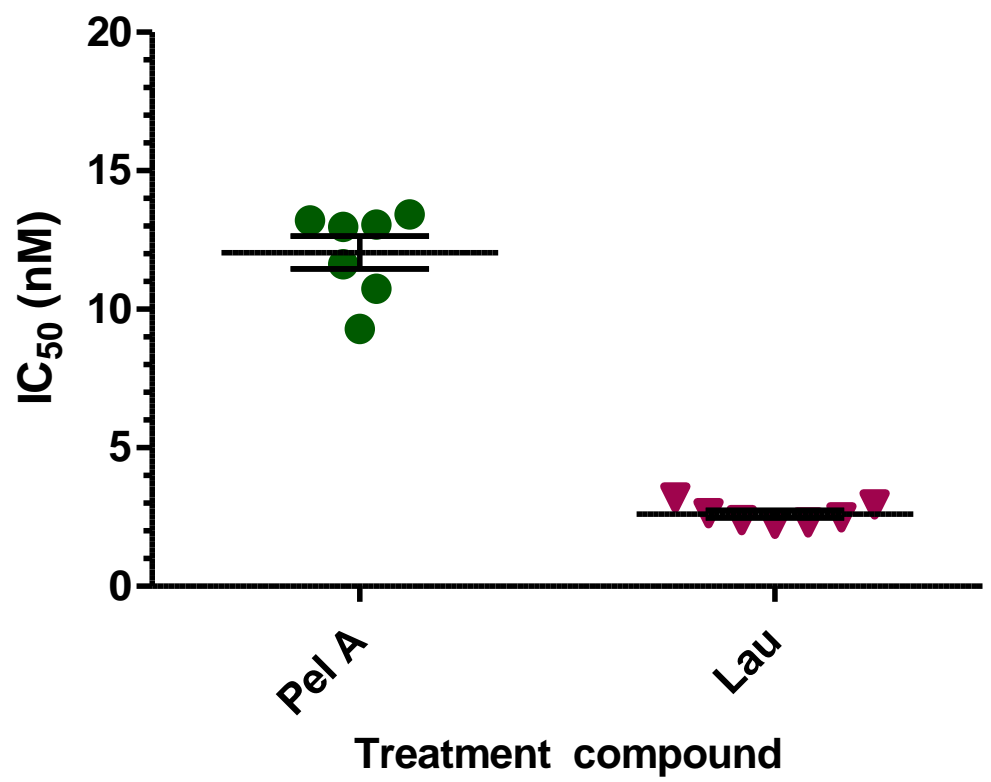

Figure 3.4.6 Concentration-response for WT tGDP- $\beta I$ transfected HEK293 cells treated with Pel $A$ and Lau.

A) Representative concentration-response curves of WT tGFP- $\beta I$-tubulin-transfected HEK293 cells treated with Pel A and Lau. Transfected cells were treated with Pel A or Lau for 72 before measurement of cell proliferation using the MTT assay as described in the methods. B) $\mathrm{IC}_{50}$ of $\mathrm{Pel}$ $A$ and Lau in $t G F P-\beta I$-tubulin transfected HEK293 cells. The $\mathrm{IC}_{50}$ for tGFP- $\beta \mathrm{I}$-tubulin transfected HEK293 cells was were determined by the MTT assay. $\mathrm{IC}_{50}$ data are presented as the mean $\mathrm{IC}_{50}$ $(\mathrm{nM}) \pm \mathrm{SEM}(\mathrm{n}=7$ biological replicates $)$. 
A)

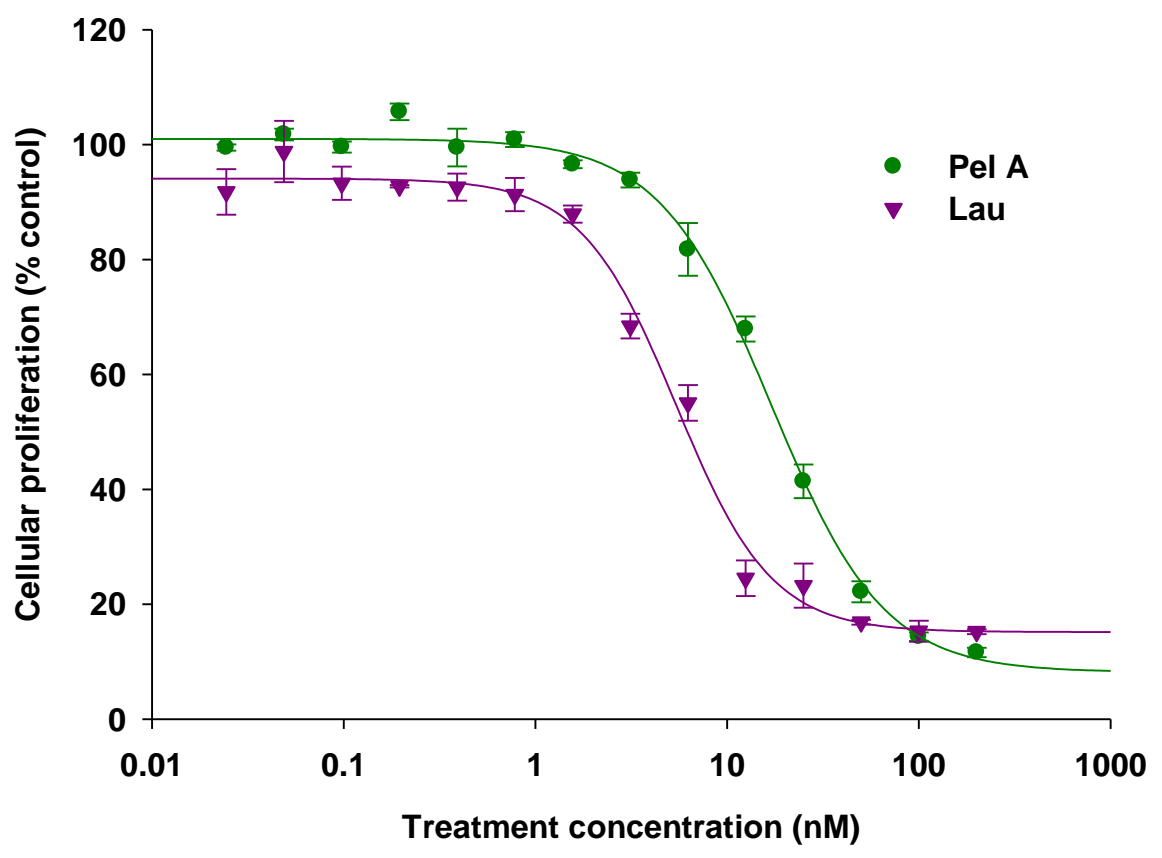

B)

IC $_{50}$ of Pel A and Lau on tGFP-D297I- $\beta$ I-tubulin transfected HEK293

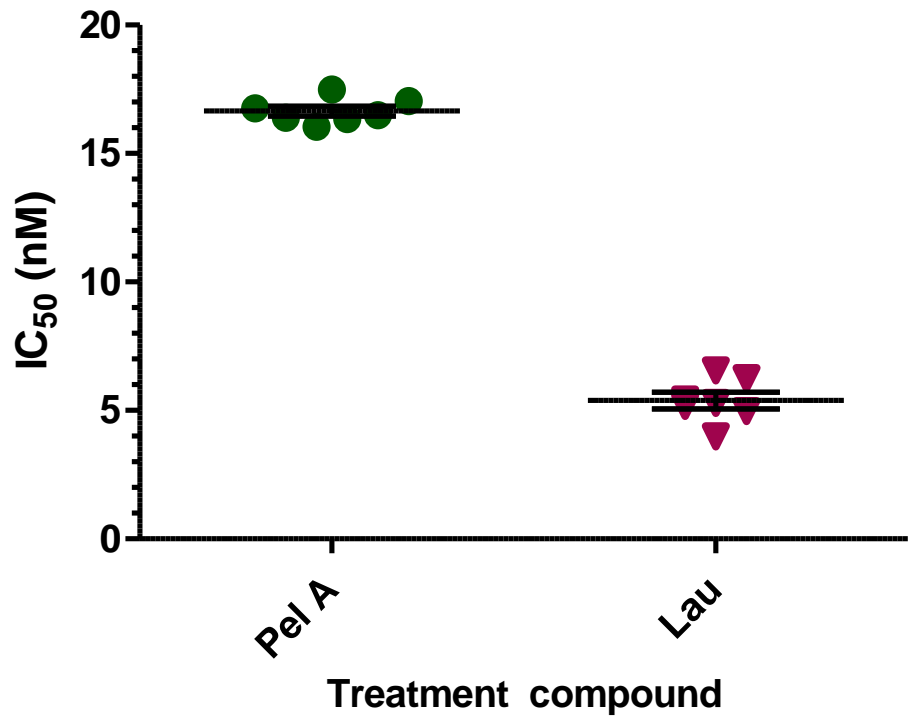

Figure 3.4.7 Concentration-response for tGDP-D297I- $\beta$ I mutant transfected HEK293 cells treated with Pel A and Lau.

A) Representative concentration-response curves of WT tGFP-D297I- $\beta I$-tubulin-transfected HEK293 cells treated with Pel A and Lau. Transfected cells were treated with Pel A or Lau for 72 before measurement of cell proliferation using the MTT assay as described in the methods. B) $I C_{50}$ of Pel $A$ and Lau in tGFP-D297I- $\beta$ I-tubulin transfected HEK293. The IC $_{50}$ for tGFP-D297I- $\beta$ I transfected HEK293 cells was determined by the MTT assay. $\mathrm{IC}_{50}$ data are presented as the mean $\mathrm{IC}_{50}(\mathrm{nM}) \pm \operatorname{SEM}(\mathrm{n}=7$ biological replicates $)$. 


\section{IC $_{50}$ of Pel A treated HEK293 transfectants}

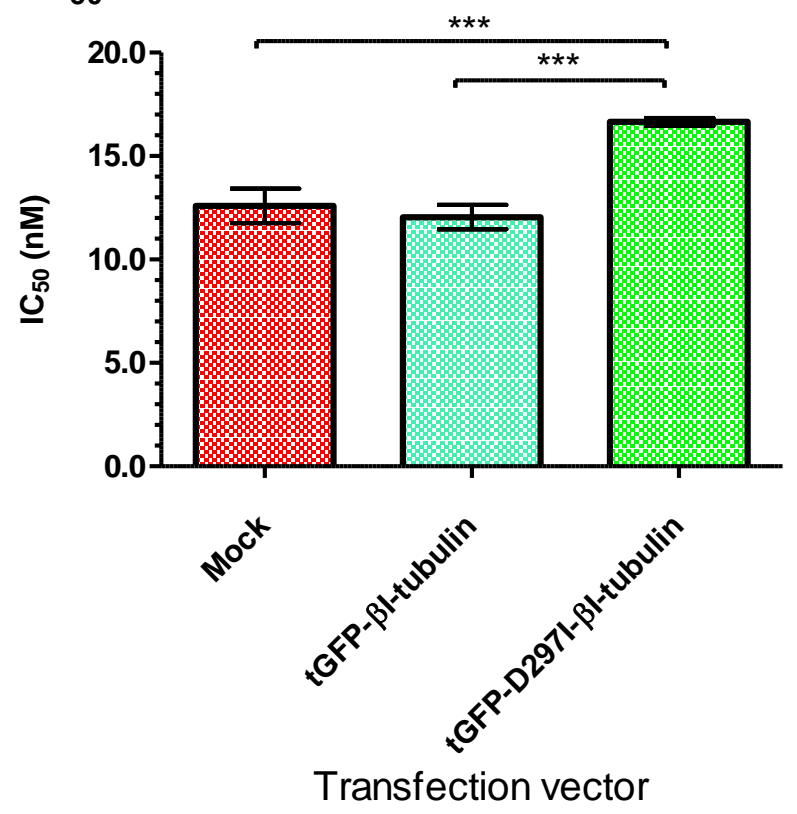

$\mathrm{IC}_{50}$ of Lau treated HEK293 transfectants

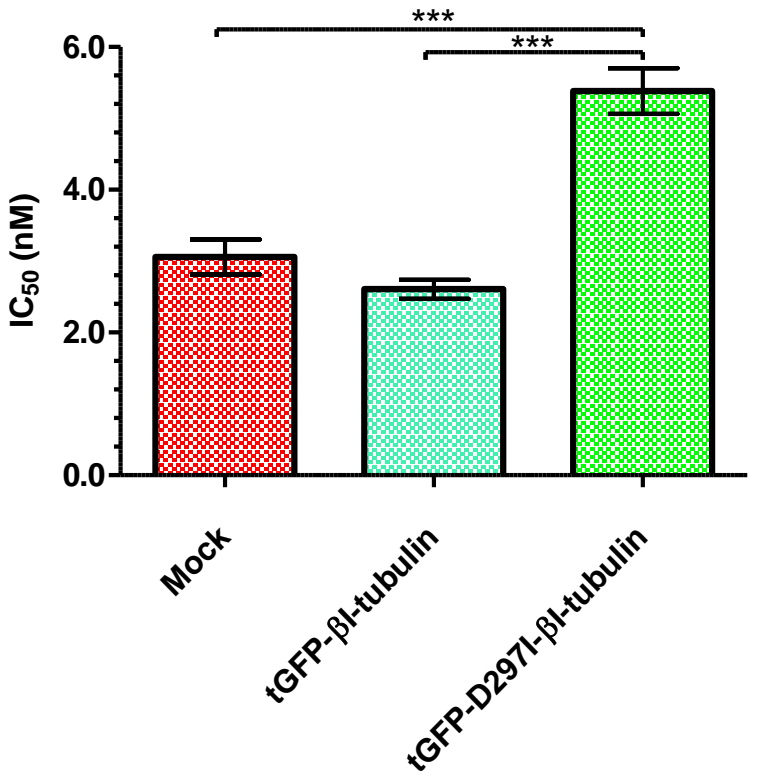

Transfection vector

Figure 3.4.8 Summary of $I C_{50}$ data for Pel A and Lau treatments of HEK293 cells.

Cells transfected with mock-control (Lipofectamine only), WT tGFP- $\beta$ I-tubulin or mutant tGFPD297I- $\beta I-t u b u l i n$ expression vectors. HEK293 expressing the mutant tGFP-D297I- $\beta I$-tubulin exhibit a small but consistent resistance to both Pel A and Lau indicating D297I mutant $\beta I$-tubulin subunit presence within the total cell population confers a resistance phenotype in response to either drug treatment (Student's t-test $* * * p<0.0001$ compared to WT transfected tubulin). Data are presented as mean $\mathrm{IC}_{50}(\mathrm{nM}) \pm \mathrm{SEM}(\mathrm{n}=7$ biological replicates). 
HEK293 cells transfected with WT tGFP- $\beta$ I-tubulin, mutant tGFP- $\beta$-tubulin or mockcontrol transfected cells were tested with Pel A and Lau using the MTT cellular proliferation assay. Mock-control and tGFP- $\beta$ I-tubulin transfected HEK293 cells showed no significant difference in response to either transfected tubulin $(\mathrm{p}=>0.05$ Student's $t$ test), however tGFP-D297I- $\beta$ I-tubulin-transfected cells demonstrated a small but highly consistent resistance phenotype to both Pel A and Lau, indicating drug-tubulin interactions may have been impacted in some capacity. The data of Figures 3.3.5 to 3.3.8 and Table 3.3.2 indicate that expression of the mutant tGFP-D297I- $\beta$ I-tubulin causes a small but consistent increase in the $\mathrm{IC}_{50}$ values for both Pel A and Lau. The changes observed in $\mathrm{IC}_{50}$ concentrations for Pel A and Lau in HEK293 cells transfected with the D297I point mutation are likely to be a result of altered drug-tubulin interactions. Disruption of the predicted hydrogen bond interaction between D297 and the O2 hydroxyl of Pel A, or the O20 hydroxyl of Lau, was originally hypothesised to impact on the molecular interaction between $\beta$ I-tubulin and these stabilising agents, thus reducing their ability to stabilise microtubules in a polymerised conformation.

\begin{tabular}{|c|c|c|c|c|}
\hline \multirow[b]{2}{*}{ Compound } & \multicolumn{3}{|c|}{$\mathrm{IC}_{50}(\mathrm{nM}) \pm$} & \multirow{2}{*}{$\begin{array}{l}\text { Resistance Ratio } \\
\text { IC50 D297I I I mutant } \\
\end{array}$} \\
\hline & $\begin{array}{l}\text { Mock- } \\
\text { control }\end{array}$ & $\begin{array}{l}\text { tGFP- } \beta I- \\
\text { tubulin }\end{array}$ & $\begin{array}{l}\text { tGFP- } \beta \text { I-D297- } \\
\text { tubulin }\end{array}$ & \\
\hline Pel A & $12.6 \pm 0.8$ & $12.1 \pm 0.6$ & $16.0 \pm 0.2 * * *$ & $1.3 \pm 0.1 * * *$ \\
\hline Lau & $3.1 \pm 0.2$ & $2.6 \pm 0.1$ & $5.4 \pm 0.3 * * *$ & $2.1 \pm 0.1 * * *$ \\
\hline
\end{tabular}

Table 3.4.2 Summary IC $C_{50}$ data for Pel A and Lau treatment of HEK293 transfection variants. Presented here are $\mathrm{IC}_{50}$ data for Pel A and Lau treatment of mock-control, tGFP- $\beta \mathrm{I}$-tubulin and tGFP-D297I- $\beta$ I-tubulin transfected HEK293 cells. $\mathrm{IC}_{50}$ for each transfection species were determined by the MTT proliferation assay as described in 3.2.1. Data are presented as the mean \pm SEM of 7 biological replicates with ***p $<0.0001$ by Student's t-test. D297I- $\beta$ I-tubulin expression within transfected cell populations results in a resistance phenotype compared to tGFP- $\beta$ I-tubulin transfected cells. No significant difference between tGFP- $\beta$ I-tubulin and mock-control transfected populations was observed $(\mathrm{p}>0.05)$. 


\subsubsection{Effect of Pel A on in situ polymerisation of $\beta$ 1-tubulin containing D297I mutant subunits}

Dynamicity within the cytoskeletal systems of cells is critical to maintaining the normal function of many cellular processes. Tubulin $\alpha-\beta$ heterodimers exist in constant flux between soluble and insoluble states as microtubules undergo polymerisation (growth) or catastrophe depolymerisation (catastrophe). Treatment of cells with microtubule-stabilising agents introduces a major disruption in normal dynamic equilibrium, causing a decrease in dynamicity and, at high concentrations, promoting an increase in the polymerised fraction of tubulin heterodimers within cells. The in situ polymerisation state of cells can be assessed quantitatively (Giannakakou, Sackett et al. 1997) as an indicator for changes in drug-tubulin interactions such as that seen in Pel A/Lau resistance of tGFP-D297I- $\beta$ Itubulin-transfected cultures. Mock-transfected (Lipofectamine only and tGFP-D297I- $\beta$ Itubulin transfected cultures were treated with Pel A as described in section 3.2.2, and the polymerised and soluble fractions of tubulin were separated by centrifugation followed by SDS-PAGE (Figure 3.3.9). Immunoblotting for $\beta$ I-tubulin revealed an interesting shift in the solubility profile of $\beta$ I-tubulin between wild type and mutant- $\beta$ I transfected samples. In the absence of Pel A, mock-transfected HEK293 cells had an average soluble $\beta$ I-tubulin fraction of $52.8 \pm 1.3 \%(\mathrm{n}=4$ biological replicates); while, tGFP-D297I- $\beta$ I-tubulin transfected cell samples contained a significantly higher soluble $\beta$ I-tubulin fraction of 73.6 $\pm 11.2 \%(\mathrm{n}=5$ biological replicates mean $\pm \mathrm{SEM}$; with unpaired Student's $t$ test*p < 0.0001 compared to the mock transfected cells). This trend was observed consistently across all three treatment concentrations. Higher concentrations of Pel A led to a higher fraction of $\beta I$-tubulin in the polymerised fraction in the WT tubulin cells. Cell populations transfected with the D297I tubulin mutant, however, displayed an altered drug-tubulin response to Pel A compared to mock-control transfected HEK293 cells, indicating a change in drug- $\beta$ I-tubulin interactions., These results suggest that the tGFP-D297I- $\beta$ I-tubulin 
expression vector makes a more major contribution than anticipated from the low (approximately 5-15\%) transfection expression levels in the HEK293 cells. Thus, the mutant tubulin may have contributed a larger pool of $\beta$ I-subunits than first thought. In order to alter the soluble fraction of $\beta \mathrm{I}$-tubulin species to this extent, quantities of mutant $\beta \mathrm{I}-$ tubulin were potentially far greater than the 5 to $15 \%$ detected by Western blot (Fig. 2.3.10). 


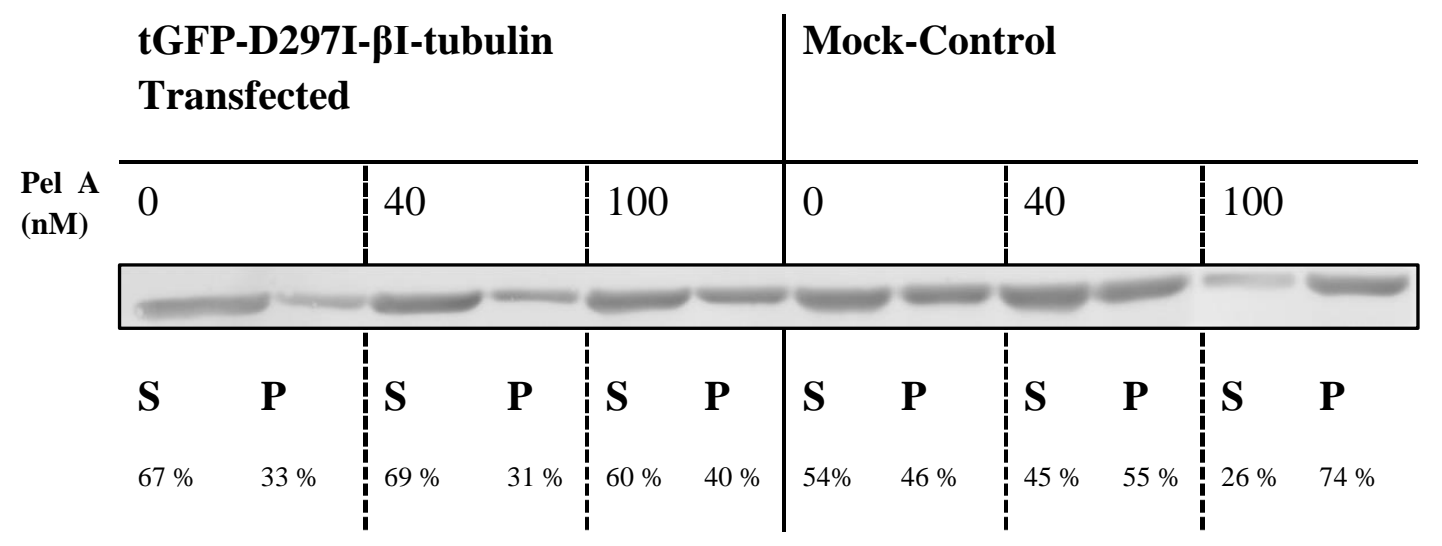

Figure 3.4.9. Representative in situ polymerisation experiment for HEK293 cells treated with Pel $A$. Presented here is a representative immunoblot from a single in situ polymerisation assay in HEK293 cells. Mutant tGFP-D297I- $\beta$ I-tubulin-transfected HEK293 cells and mock transfected cells were treated for $16 \mathrm{~h}$ with 0 , 40, or $100 \mathrm{nM}$ Pel A. The soluble (S) $\beta$ I-tubulin was electrophoresed alongside the corresponding polymerised fraction $(\mathrm{P})$.

\section{In situ polymerisation of transfected HEK293}

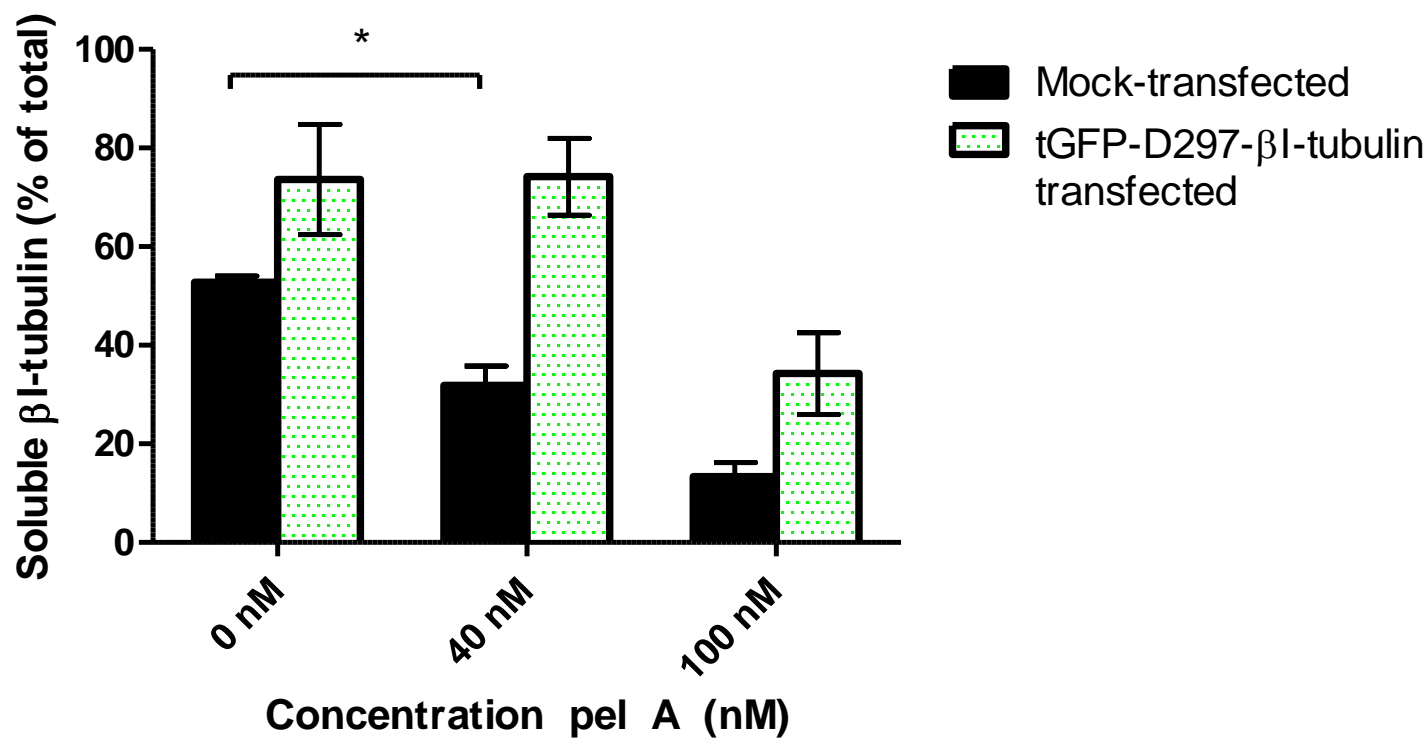

Figure 3.4.10 In situ polymerisation of HEK293 cells. This figure provides a summary of in situ polymerisation of HEK293 cells transfected with the tGFP-D297I- $\beta$ I-tubulin mutation plasmid after $16 \mathrm{~h}$ treatment with Pel A. Data are presented as the mean $\pm \mathrm{SEM} \%$ of the total $\beta \mathrm{I}$-tubulin in the soluble fraction from 5 biological replicates $(* \mathrm{p}=<.05)$. 
In the mock transfected cells, soluble $\beta$ I-tubulin content within samples decreased in response to higher Pel A concentrations, indicating a greater abundance of $\beta \mathrm{I}$-tubulin subunits stabilised within polymerised microtubules (Figure 3.4.10). tGFP-D297- $\beta$ Itubulin transfected HEK293 cells displayed an altered profile of $\beta I$-tubulin polymerisation compared to the mock-transfected cells, with a higher abundance of the soluble tubulin fraction at all drug concentrations. No significant difference was found in the portion of soluble $\beta$ I-tubulin in tGFP-D297- $\beta$ I-tubulin transfected samples at 0 or $40 \mathrm{nM}$ Pel A. Contrastingly, a significant change was seen in the mock-control between 0 and $40 \mathrm{nM}$ Pel A (Student's $t$-test, $\mathrm{p}=.016$ ) indicating the presence of D297I $\beta$ I-tubulin in transfected cells was altering the ability of Pel A to induce polymerisation. Both mock-transfected control and mutant transfected HEK293 demonstrate a significant decrease in the soluble $\beta$ I-tubulin fraction after treatment with $100 \mathrm{nM}$ Pel A, however the difference in the percentage change within each group did not reach significance when compared to each other.

\subsubsection{Secondary re-assessment of D297I mutant $\beta 1$-tubulin presence in HEK293}

The WT and mutant tGFP- $\beta$ I-tubulin content of transfected HEK293 cells as assessed by Western blot in section 2.3.6 was considerably lower than the expected values based on preliminary experiments in the $1 \mathrm{~A} 9$ cell line and similar work carried out in our laboratory using HEK293 cells (Kanakkanthara, A. unpublished). A number of independent observations made here in concentration-response assays, confocal imaging, and immunoblotting suggest the total tGFP- $\beta$ I-tubulin content of transfected cell samples may have in fact been more abundant than what was quantified by Western blot (5-15\% of total $\beta$ I-tubulin in the cell) in section 2.3.6. In a separate experiment, immunoblotting against exogenous tubulins was carried out by targeting the tGFP fusion construct on the exogenous $\beta I$-tubulin, rather than an epitope present within $\beta$ I-tubulin itself. Only a single blot of this 
type was carried out, though samples present within the preparation came from a range of different transfection experiments collected over the duration of this research. Due to differences in both antibody fidelity and concentration between $\beta I$ and tGFP-targeting primary antibodies, a relative quantification of mutant expression was not the intended purpose of this Western blot. All transfected samples were positive for a strong tGFP signal, indicating tGFP antigen was present in approximately equal density in each of the mutant tGFP- $\beta$ I-tubulin samples assessed. The intensity of this fluorescence of this GFP signal suggests tGFP antigen recognition sites were abundant in the samples, however; any effort to quantify mutant $\beta \mathrm{I}$-tubulin expression is not directly comparable to the signals detected in section 2.3.6. Thus, this immunoblot serves only as a small piece of indirect evidence questioning the validity of the low abundance of mutant- $\beta$ I-tubulin detected in section 2.3.6 using a $\beta I$-tubulin specific primary antibody against these fusion sequences. 


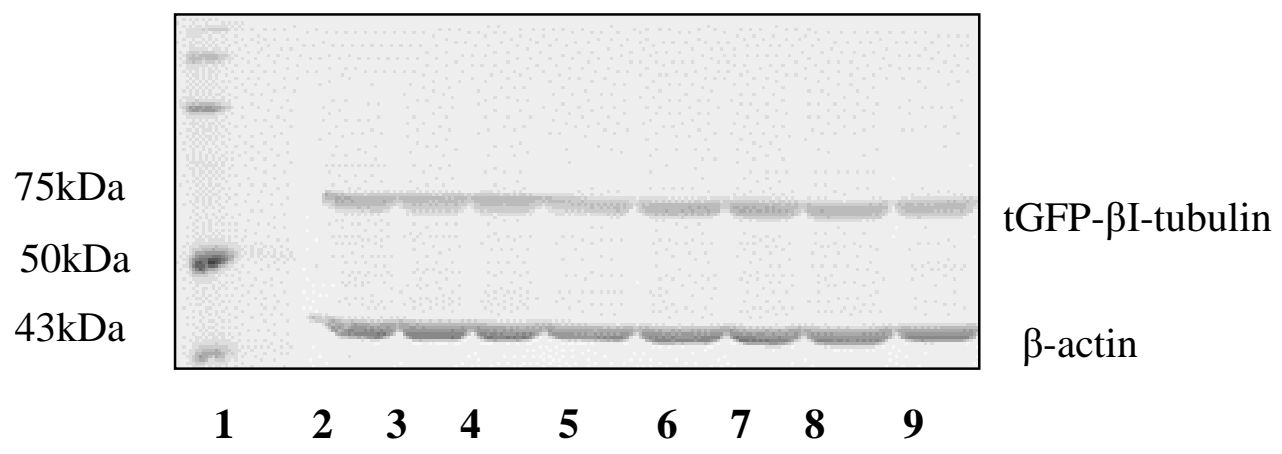

Figure 3.4.11 Secondary reassessment of tGFP presence in tGFP-D297I transfected HEK293. Presented here is an immunoblot detecting tGFP within tGFP-D297I-BI-tubulin transfected samples in HEK293 cells. Lane 1: Molecular weight ladder. Lanes 2-9: tGFP-D297- $\beta$ I-tubulintransfected samples corresponding to 8 individual concentration-response experiments at the $144 \mathrm{~h}$ time point. $\beta$ I-actin serves as a loading control in each of the sample lanes, and a tGFP-positive signal was detected within all samples. The intensity of the tGFP signal was strong in all lanes, indicating tGFP- $\beta$ I-tubulin expression was relatively stable across these 8 particular transfection experiments. The signal present is comparatively stronger than that of tGFP- $\beta$ I-tubulins detected as described in section 2.3.6, however it is not possible to directly compare these signals in a quantitative manner. 


\subsubsection{Discussion}

The proliferative behaviour of tGFP-D297I- $\beta$ I-tubulin transfected HEK293 cells in PTX and IXA as an indicator of mutant $\beta I-t u b u l i n$ fidelity in cellular processes

Before any investigation into the specific role of the D297I point mutation in the predicted $\beta$ I-tubulin binding association with Pel A or Lau, it was critical to determine that the introduction of mutant $\beta$ I-tubulin subunits into HEK293 cells did not alter the proliferative phenotype of these cells treated with drugs targeting the taxane region of $\beta$-tubulin. The introduction of irregular $\beta$ I-tubulin subunits, including in our case, subunits with tGFPfusion tags, had potential to significantly alter the normal concentration-response of cells to treatment with PTX or IXA by impacting global cytoskeletal functions. In this study, a thorough evaluation of proliferation characteristics in transfected HEK293 cells following treatment with PTX and IXA revealed no significant differences between the concentrationresponses of cells containing tGFP-D297- $\beta$ I-tubulin, tGFP-WT $\beta$ I tubulin or mock transfected cultures. This was expected as the mutated amino acid was a long distance away from the taxoid binding site. The concentration-response $\mathrm{IC}_{50}$ values determined for cells treated with PTX or IXA were consistent with other reports of low nanomolar concentration activities of these drugs in untransfected cells (Hopper-Borge, Chen et al. 2004, Chen, Sun et al. 2012, Kathawala, Wei et al. 2014) (Kanakkanthara, A. Victoria University of Wellington. Unpublished data.), although PTX and IXA concentration-response data has been more fully explored in other cell lines, primarily those derived from cancerous tissue such as 1A9 cells (Giannakakou, Sackett et al. 1997, Dumontet, Jordan et al. 2009).

Drug competition studies carried out in early stages of Pel A and Lau characterisation revealed a distinct mode of action from that of traditional stabilising agents with binding associations within the well-characterised taxoid binding-region of $\beta$ I-tubulin (Nogales, 
Whittaker et al. 1999). Pel A was found to not compete PTX, yet was displaced from binding associations by Lau, the conclusion being that Pel A and Lau share the same or an overlapping binding region, distinct from the taxoid site (Gaitanos, Buey et al. 2004). Lau had been shown previously to not compete with PTX for binding to tubulin (Pryor, O'Brate et al. 2002). Multiple modelling studies published in support of a distinct Pel A/Lau binding site have characterised this unique binding region over time (Huzil, Chen et al. 2007, Huzil, Chik et al. 2008, Bennett, Barakat et al. 2010, Prota, Bargsten et al. 2013, Prota, Bargsten et al. 2014).

The introduced D297I point mutation focused on within this study did not affect cellular proliferation following treatment with the taxoid site binding drugs PTX or IXA. This suggests the effects of the introduced point mutation are likely localised to a discrete region surrounding amino acid residue 297 within the Pel A and Lau binding region. The tGFPfusion tag expressed constitutively with the plasmid-encoded $\beta I$-tubulin sequence was tolerated within heterodimer polymerisation, supported by this same lack of concentrationresponse shift between untransfected and WT tubulin transfected cultures after treatment with PTX or IXA.

Amino acid polymorphisms as products of natural or artificially introduced mutations can cause significant alterations in protein function, particularly in cases whereby an alteration takes place in critical peptide sequences within secondary or tertiary folding motifs, or sites of catalytic activity. Additionally, mutations in the unique carboxy-terminal sequences of $\beta$-tubulin isotypes can affect post translational modifications, thus altering regular interactions with microtubule associated proteins (MAPs) (Luduena 1998, Verdier-Pinard, Pasquier et al. 2009). A multitude of $\beta I$-tubulin point mutations have been identified within the literature that impact on polymerisation, stability or function of microtubules (Hari, 
Loganzo et al. 2006, Kavallaris 2010). This has implications with regard to therapeutic options available for treating disease states with drug resistance phenotypes caused by specific alterations to drug binding associations within microtubule targets.

A single mutation can lead to global structural changes at long distances from the site of the mutation as the $\beta$ I-tubulin peptide sequence adjusts to altered electrostatic, hydrophobic, and steric interactions, adopting a variant conformation with more favourable thermodynamic properties. This alteration may disrupt electrostatic interactions between associated protein complexes (Sheinerman, Norel et al. 2000), which is of concern when working with a protein such as $\beta$ I-tubulin that is composed of heterodimer subunits. The D297I point mutation in this investigation was designed with consideration given to potential destabilizing interactions to reduce the likelihood that an introduced change would severely impact the global structure of tubulin, but would rather only alter electrostatic interactions between PelA or Lau and amino acid 297. This design was accomplished by using molecular modelling software analysis of the Pel A/Lau binding site on $\beta 1$-tubulin (Assoc. Prof Paul Teesdale-Spittle, personal communication). Subsequent to the design and construction of the mutant tubulin used in the present study, the x-ray crystallography model of Pel A and Lau bound to tubulin became available (Prota, Bargsten et al. 2014).

The predicted hydrogen bonding interaction between D297 and Pel A or Lau based on the X-ray crystallography analysis of Prota, Bargsten et al. (2014) suggested that a substitution of aspartic acid by isoleucine would affect the interaction of Pel A and Lau with the binding region on tubulin. Isoleucine is a small, non-polar amino acid with similar steric characteristics to aspartic acid; however, isoleucine lacks the polar functionality of a hydroxyl group within the side chain. This particular substitution would disrupt a favourable hydrogen bonding interaction between the $\mathrm{O} 2$ atom of Pel $\mathrm{A}$ and the associated 
hydroxyl of the aspartic acid side chain at position 297 of the $\beta$ I-tubulin peptide sequence. Similarly, the O20 atom of the Lau side chain should be able to form a hydrogen bond with the hydroxyl group of the aspartic acid side chain. Thus, the isoleucine replacement of aspartic acid at residue 297 of tubulin should disrupt the bond associations with both Pel A and Lau without introducing any widespread conformational change, as the aspartic acid and isoleucine are relatively small amino acids. Thus, localised steric hindrance would be avoided and there would be no propagation of these changes through the tertiary $\beta I$-tubulin structure. The results presented in this investigation demonstrated that HEK293 cells transfected with tGFP-D297I- $\beta$ I-tubulin did not exhibit large-scale changes in proliferative phenotype following transfection, indicating minimal disruption in tertiary protein structure or function. The D297I point mutation allows for further study into Pel A or Lau binding interactions in the region, with any alterations in drug sensitivity attributed directly to the disruption of discrete hydrogen bonding interactions and not non-specific changes in global conformation.

\section{tGFP-D297I- $\beta$ I tubulin-transfected HEK293 cells show resistance to Pel A or Lau inhibition of proliferation}

The D297I point mutation was demonstrated to have no impact on proliferation of transfected cultures treated with taxoid-site targeting drugs. This was consistent with our hypothesis, and validated the use of concentration-response data from PTX and IXA treatment as a control for global alterations in $\beta$ I-tubulin function as a result of mutation or the presence of the tGFP fusion-tag. Data were collected from 7 biological repeats, in duplicate - a total of 14 individual measurements - for each of the conditions, tGFP- $\beta$ Itubulin, tGFP-D297I- $\beta$ I-tubulin or mock-transfected HEK293 cells under each of the 4 drug treatments. This large dataset showed consistent responses and allowed even small changes in proliferative response to be detected as a result of D297I-tubulin presence in 106 
comparison to cell populations with wild type $\beta$ I-tubulin alone. Evidence presented within Chapter 2 of this thesis suggested that the mutant $\beta$ I-tubulin presence within transfected cultures was relatively low; however, the robust nature of the data collected for mocktransfected and tGFP- $\beta$ I-tubulin transfected cells allowed the detection of a small drug resistance shift in D297I- $\beta$ I-tubulin-expressing HEK293 cells with treatment by either Pel A or Lau.

In cultures treated with Pel $\mathrm{A}$, the $\mathrm{D} 297 \mathrm{I}$ point mutation conferred a $1.3 \pm 0.1$ fold increase in the $\mathrm{IC}_{50}$ of tGFP-D297I- $\beta$ I-tubulin transfected HEK293 cell cultures relative to the mock-transfected control. tGFP- $\beta$ I-tubulin transfected cultures demonstrated no significant change in concentration response, consistent with our hypothesis that expression of tGFPWT- $\beta$ I-tubulin should not impact binding properties within the target region of Pel A. These fusion-tagged subunits are expected to behave in a manner equivalent to endogenous WT $\beta$ I-tubulin. In the case of the D297I-mutant tubulin, the small but highly consistent drug resistant phenotype obtained provided support for disruption of Pel A binding interactions in the region of the point mutation. Within the main macrolactone structure of Pel A, the $\mathrm{O} 2$ hydroxyl likely functions as an enhancer of stability for Pel A binding within the $\beta \mathrm{I}-$ tubulin Pel $\mathrm{A} / \mathrm{Lau}$ binding pocket. The replacement of aspartic acid with isoleucine in our transfected model appears to have disrupted this predicted interaction within the mutant homodimers, leading to an altered state of molecular interactions in the binding region. The predicted mechanism of Pel A induced microtubule-stabilisation (Prota, Bargsten et al. 2014) suggests the binding association of Pel A induces multiple conformational shifts within the helical segment of residues Gln294 to Phe296, leading to a stabilized one-turn extension of the $\mathrm{H} 9$ helix. This in turn, promotes the formation of water-mediated hydrogen bonding between PelA and the H9 amino acid residues Gln294 
and Leu275 and additional stabilisation by hydrogen bonding to residue Asn300. Combined, these changes would promote a re-organisation of the M-loop substructure in $\beta \mathrm{I}$-tubulin.

The M-loop is an essential structural element within $\beta$ I-tubulin and is directly involved in the association of complete $\alpha-\beta$ protofilaments during polymerisation mechanics of the microtubule (Nogales, Whittaker et al. 1999, Li, DeRosier et al. 2002, Prota, Bargsten et al. 2013). Pel A-induced conformational change within the M-loop is predicted to reduce the thermodynamic requirements for interactions between neighbouring protofilaments, leading to enhanced polymerisation dynamics and stability in extended microtubule polymers. Any loss of these interactions would therefore decrease the ability of Pel A to cause microtubule-stabilisation, conferring resistance to the cell expressing the D297I mutant tubulin. Thus, the small shift in $\mathrm{IC}_{50}$ of Pel A is likely a result of a decrease in the compounds ability to induce organisational changes within the M-loop of an individual $\beta I-$ tubulin subunit in the presence of the D297I point mutation. This may result in a reduced level of drug-enhanced interprotofilament associations throughout the macrostructure of the microtubule.

With Lau, HEK293 cells transfected with tGFP-D297I- $\beta$ I-tubulin were found to have a 2.1 \pm 0.1 fold increase in $\mathrm{IC}_{50}$ value relative to the mock-transfected control. tGFP- $\beta$ I-tubulin transfected cultures again demonstrated no significant alteration in concentration-response, providing further support for the retention of normal function of tGFP- $\beta$ I tubulin subunits. The highly consistent shift reflected in concentration-response data of cultures transfected with tGFP-D297I- $\beta$ I-tubulin reinforces the role of the D297 residue in the formation of Lau binding interactions. Hydrogen bonding interactions by D297 with the O20 hydroxyl group in the side chain of Lau, as predicted by Prota et al., 2014, are disrupted in mutant $\beta I$-tubulin subunits presenting the non-polar isoleucine residue, leading to a reduction in compound 
binding stability within the region. This would reduce the ability of Lau to induce organisational changes in the M-loop of $\beta$ I-tubulin and reduce the extent of the enhanced protofilament interactions predicted to occur in wild-type $\beta$ I-tubulin by treatment with Lau. The higher fold-change of Lau resistance relative to Pel A in cultures presenting with the D297I mutation suggest D297 has a more central role than Pel A in stabilising the binding association of the compound and inducing M-loop re-organisation.

\section{The introduction of tGFP-D297- $\beta$ I-tubulin reduces intracellular polymerisation in the presence of Pel A}

To determine whether the presence of mutant D297- $\beta$ I-tubulin subunits affects Pel Ainduced tubulin polymerisation, an in situ assessment of tubulin solubility was carried out in transfected HEK293 cells. Induced microtubule polymerisation was evaluated after $16 \mathrm{~h}$ Pel A treatment by examining the relative abundance of $\beta I$-tubulin in the soluble and polymerised tubulin fractions. In mock-transfected cells, treatment with $40 \mathrm{nM}$ Pel A resulted in a $20.9 \pm 4.1 \%$ reduction in the abundance of $\beta$ I-tubulin detected within the soluble protein fraction compared to untreated cells. This is the result of a shift in polymerisation dynamics favouring the formation of insoluble tubulin polymers, stabilized in the presence of Pel A. In tGFP-D297I- $\beta$ I-tubulin transfected cells, this same shift polymer formation was not observed, with no significant difference in the portion of $\beta I-$ tubulin detected in the soluble fractions between treatments at 0 or $40 \mathrm{nM}$ Pel A. This indicates that the D297I point mutation present within a portion of $\beta$ I-tubulin subunits interfered with the level of Pel A-induced microtubule-assembly seen in mock-transfected cultures. A strong correlation exists between this observation and the higher $\mathrm{IC}_{50}$ value for Pel A, suggesting D297I- $\beta$ I-tubulin subunits induce resistance to Pel A. 
At a concentration of $100 \mathrm{nM}$, Pel A induced a significant level of tubulin-assembly in both the mock and tGFP-D297I- $\beta$ I-tubulin transfected cultures. The IC $_{50}$ values for inhibition of growth of mock and D297I mutant transfected cultures were determined to be $12.6 \pm 0.8$ and $16.0 \pm 0.2 \mathrm{nM}$, respectively. It is not surprising, therefore, that a significant level of tubulin polymerisation was observed in both cultures as the concentration used of $100 \mathrm{nM}$ is several-fold above the $\mathrm{IC}_{50}$. The $\mathrm{D} 297 \mathrm{I}$ point mutation is likely to alter the binding affinity of Pel A in the predicted binding region, reducing the level of organisation induced in the M-loop of $\beta I-$ tubulin.

Surprisingly, the percentage of soluble $\beta$ I-tubulin in untreated cells varied significantly between the tGFP-D297I- $\beta$ I-tubulin and mock-transfected cells $(73.6 \pm 11.2 \%$ and $47.8 \pm$ $6.0 \%$ respectively). Transfection with plasmid-encoded $\beta$ I-tubulins appeared to reduce the number of polymerised microtubules in the cells; however as an alternative hypothesis, this observation could support the idea that higher levels of plasmid-encoded mutant $\beta \mathrm{I}$-tubulin are present than was detected by Western blot. Protofilaments made up of $\alpha$ - $\beta$-tubulin heterodimers are arranged in a highly organised structure in the dynamic process of forming the polymerised microtubule structure. Before soluble (S) $\beta$ I-tubulin can shift to the insoluble tubulin fraction $(\mathrm{P})$, it must associate with $\alpha$-tubulin in a 1:1 stoichiometry to form the heterodimer. In conditions of $\beta$ I-tubulin overexpression, the relative abundance of $\alpha$ tubulin monomers does not satisfy the requirements for complete heterodimer formation, preventing a fraction of $\beta$ I-tubulin from contributing to microtubule polymers. This is put forward as a proposed explanation for the observation of a greater proportion of insoluble $\beta I$-tubulin seen across all three concentrations of Pel A. Interestingly, the bands corresponding to a molecular weight of $75 \mathrm{kDa}$ (GFP-tubulin) were not observed within any in situ polymerisation experiments, although lysates were prepared from highly 
fluorescent, transfected cultures as assessed by microscopy prior to sample preparation. This is not consistent with the detection of tGFP- $\beta$ I-tubulins by immunoblotting described in Chapter 2 of this investigation. The method of in situ lysate preparation for the analysis of tubulin polymerisation profiles varies significantly from the standard Western blot procedure used to detect plasmid-encoded expression levels, suggesting that at some stage during the in situ polymerisation process, it is possible that the tGFP peptide sequence is lost from some or all of the exogenous transfected $\beta$ I-tubulins; hence, the absence of the 75 $\mathrm{kDa}$ GFP-tubulin band. To prevent this occurring, the electrophoresis sample was not boiled prior to loading on the polyacrylamide gel, but some other step in the preparation of the samples for electrophoresis may be involved.

\section{Independent assessment of the presence of tGFP in transfected cultures supports the} need to quantify mutant $\beta I$-tubulin expression by an alternate method

As a brief check on tGFP protein in the electrophoretic gels, eight tGFP-D297I- $\beta$ I-tubulin transfected cell sample lysates from the $144 \mathrm{~h}$ time point of concentration-response studies were analysed by immunoblotting for the presence of tGFP specific antigens using an antitGFP primary antibody and a fluorescent secondary antibody. Bright signals at $75 \mathrm{kDa}$ were detected, corresponding to the presence of tGFP- $\beta$ I-tubulin species as expected within the lysate. However, there was no band seen at $25 \mathrm{kDa}$ to indicate loss of the GFP tag during the preparation of the sample for electrophoresis; one hypothesis for the low abundance of mutant protein detection by western blotting. The same fluorophore-conjugated secondary antibody was applied as in earlier immunoblotting experiments, but appeared to have a surprisingly strong signal with primary antibodies targeted to tGFP antigens as opposed to $\beta$ I-tubulin. The specific activities of each primary antibody, along with variation in the final concentration of the reagents, prevents direct comparison of the fluorescence-signal output 
within each experiment type; however, it is important to acknowledge the stark contrast seen between experiments. This observation supports the idea that mutant $\beta$ I-tubulins may have been present in higher abundance than detected by Western blot using primary anti$\beta I-t u b u l i n$ antibodies. This supports a need for future application of a proteomics-based approach to quantify mutant $\beta$ I-tubulin expression as discussed in Chapter 2 . The small resistance phenotype seen with Pel A and Lau induced by the presence of the D297I mutation in exogenous $\beta$ I-tubulin clearly indicates an altered state of drug-binding; however, the significance of this altered drug binding needs to be better correlated to the relative abundance of the mutant residues. If the mutant D297I residue is in fact only present at very low levels, as suggested by the Western blot of lysates from transfected cells, then the altered drug-binding affinity of this mutation may induce a far greater change in drug-sensitivity to Pel A or Lau when present at higher levels in the cells.

\section{Aspartic acid 297 of $\beta$ I-tubulin has a functional role in Pel A and Lau binding- associations}

Publications on various tubulin point mutations conferring drug-resistance phenotypes are widespread in literature, covering both mammalian and yeast models. Cancers resistant to traditional taxoid-targeted microtubule-stabilisers do so through a range of mechanisms including the upregulation of drug efflux processes (Gottesman 2002), adaptation of tubulin isotype content (Burkhart, Kavallaris et al. 2001) or point mutations that directly impact compound binding affinities (Giannakakou, Sackett et al. 1997, Begaye, Trostel et al. 2011, Kanakkanthara, Wilmes et al. 2011). By modelling $\beta$ I-tubulin point mutations, we saught to elucidate the discrete molecular mechanics of small molecule interactions within their targets and the ability of these molecules to induce changes in microtubule polymerisation and depolymerisation. By defining the relative importance of particular drug-amino acid 
interactions, the efficacy of small molecules may be greatly enhanced by rational designfocused synthetic chemistry.

Historically, microtubule-targeted agents have served as critical front line therapeutics in the treatment of hyper-proliferative disease states such as those seen in cancers. These chemotherapeutics will maintain clinical relevance until further advances in the biomedical sciences deliver even more targeted clinical solutions for the deeper, underlying cause of these diseases. Until such time, any insight into the mechanisms by which current therapeutic strategies may be improved are of high value. Although clinical testing has not yet begun for Pel A and Lau, the Pel A/Lau binding region of $\beta \mathrm{I}$-tubulin represents a valuable opportunity for the development of potential therapeutics, especially for use in cancers with drug-resistance phenotypes against the compounds currently in clinical application. Pel A is a poor substrate for P-glycoprotein drug efflux pumps and avoids the complication of drug-resistance conferred by point mutations in the taxoid binding-region of $\beta$ I-tubulin (Gaitanos, Buey et al. 2004). Likewise, Lau shares these characteristics in cell lines with drug resistance phenotypes (Mooberry, Tien et al. 1999, Pryor, O'Brate et al. 2002). Recent advances in the characterisation of the binding region shared by these compounds provides the necessary starting point for biological verification of drug-target interaction mechanics.

Amino acid D297 was predicted by x-ray crystallography to serve a functional role in the binding association of Pel A and Lau to $\beta$ I-tubulin (Prota, Bargsten et al. 2014). This study has provided biological evidence in support of this prediction through various measurement parameters, and has confirmed a functional role for D297 in the binding association of both 
Pel A and Lau during induced microtubule-assembly. Although this role has been established, the significance of the D297 amino acid amid a number of other predicted binding interactions remains unclear. Within our laboratory, we seek to expand investigations into the role of specific amino acid residues, with preliminary investigations underway into Q293, V335, N339, Y342, Y312, F296 (Kanakkanthara, Sundberg Carson, unpublished data) - residues predicted to alter drug-protein interactions with either Pel A or Lau. Further study of these predicted binding interactions is required in order assign a hierarchical weighting to the importance of each during the rational design of new synthetic analogues. 


\section{Chapter 4: Overall discussion and future directions}

\subsubsection{Closing remarks}

The unique binding region of $\beta$-tubulin associated with novel MSAs Pel A and Lau has been thoroughly characterised as a novel pharmacophore through a large number of studies carried out over the last decade. This region represents a significant alternative to the taxane binding region for the development of novel MSAs, as compounds like Pel A and Lau demonstrate potentially advantageous properties in overcoming some of the clinical limitations of currently available MTAs. In order to continue advancement in the study of compounds that associate to this region, research must look toward the generation of a complete, mechanistic basis of understanding in the specific drug-residue interactions that lead to enhanced polymerisation dynamics in microtubules with these novel compounds. With these insights, the synthesis of highly specific molecules with fewer off-target interactions may finally come to fruition, improving medical outcomes for those affected by cancers that previously, may have lacked efficacious chemotherapeutic options.

To continue our progress towards these goals, current research directions include a move towards highly specific investigations into the role of individual amino acid residues in the binding region in order to establish the functional significance of each interaction in the promotion of microtubule-assembly. Mutagenesis serves as a valuable tool for this purpose, and allows the examination of small, introduced changes in a protein target, such as $\beta$ tubulin, to be assessed at the level of individual amino acids for perturbations in normal drug-responses. Classically, randomly introduced mutations conferring drug-resistance phenotypes to Pel A and Lau have been incredibly valuable in further characterisation in the binding region of these compounds (Begaye, Trostel et al. 2011, Kanakkanthara, Wilmes et al. 2011, Kanakkanthara, Eras et al. 2014), however the random nature of introduced genetic mutations in the presence of a drug or other mutagen can lead to drug- 
resistance phenotypes through mechanisms other than the introduction of a discrete mutation in the protein target of interest. Recently, the genetically amenable nature of $S$. cerevisiae has been utilised in a site-directed mutagenesis approach, finding introduced point mutations A298T, R308H, V335W and N339L in a humanised yeast $\beta$-tubulin sequence confer resistance to Pel A (Hanna, Maass et al. 2014). These particular mutations are located within the binding pocket of Pel A, and show that disruptions at these specific sites reduces drug-induced microtubule assembly. This puts these particular residues forward as candidate effectors of drug-binding; useful knowledge for developing druganalogues with functional enhancements to these residues.

In the present study, we sought to move the investigation of single amino acid interactions with Pel A and Lau forward into mammalian cell lines, where physiological interactions may be more representative of how these compounds affect human cells. Emerging HDXMS data (Huzil, Chik et al. 2008, Khrapunovich-Baine, Menon et al. 2011), along with computational (Bennett, Barakat et al. 2010, Nguyen, Xu et al. 2010, Churchill, Klobukowski et al. 2014) and crystallographic (Prota, Bargsten et al. 2014) studies have generated insights that have allowed for the rational selection of individual amino acids to study, such as D297 as in the current investigation.

By introducing $\beta \mathrm{I}$-tubulin containing the mutant D297I substitution to HEK293 cells within a transfected cell population, this study demonstrated a small but highly consistent drug resistance effect in these cells to both Pel A and Lau relative to tGFP- $\beta$ I-tubulin transfected and WT HEK293 cells. Primary investigations revealed that the tGFP- $\beta$ I-tubulin and tGFPD297I-tubulin mutant proteins were present in relatively low abundance in transfected HEK293 cultures, however populations with this mutation present were more resistant to Pel A and Lau. This raised questions regarding the true nature of mutant protein abundance 
as discussed in earlier chapters, and warrants further assessment by alternative quantitative methods in order to better understand the significance of these observed changes in concentration-response of D297I- $\beta$ I-tubulin transfected cells treated with either Pel A or Lau.

Transfected cultures of both tGFP- $\beta$ I-tubulin or tGFP-D297I-tubulin exhibited no significant concentration-response alterations under treatment with PTX or IXA, suggesting the biological function of mutant $\beta \mathrm{I}$-tubulin subunits was not significantly different to that of WT $\beta$ I-tubulin, and introduced mutations did not significantly hinder normal biological function through global 3-D conformational change in these proteins. tGFP-tagged $\beta I$-tubulin subunits were observed while incorporated within polymerised microtubule structures by live cell confocal microscopy, demonstrating these mutant components were actively involved in typical microtubule functions. The observed concentration-response shift of D297I substitution mutant-transfectants under treatment with Pel A or Lau was a small fold change, however this is may be the result of incomplete distribution of mutant D297I- $\beta$ I-tubulin subunits throughout the total cell population. The data presented within this thesis suggest that the introduction of mutant D297I $\beta$ I-tubulin by means of transfection allows for retention in dynamic-instability at a higher concentrations of Pel A or Lau. This implicates amino acid D297 of $\beta$ I-tubulin as likely to be directly involved in the normal binding association of both Lau and Pel A in the region of the mutation.

\subsubsection{Future Directions}

In this investigation, the introduction of the mutant $\beta$ I-tubulin isotype by means of transient transfection allowed the functional role of this particular mutation in Pel A and Lau binding to be evaluated, as seen through changes observed in concentration-response studies. As 
the shift in response was a small fold-change, any further studies into the functional role of individual amino acids, if carried out utilising similar methodologies, have a need for adaptations to enhance the resolution of any detectable changes in concentration-response. Variations in the approach designed to improve the abundance of mutant $\beta I$-tubulin, or even replace endogenous $\beta$ I-tubulin contributions entirely may result in the generation of highly resistant cells, such as those generated through clonal selection in the presence of drug (Begaye, Trostel et al. 2011). These improved models are likely to demonstrate larger shifts in concentration-response to drugs of interest. An improved model may allow for greater insight into the hierarchical nature of each amino acid-drug interactions importance during induced-stabilisation, guiding strategic design of future Pel A/Lau compound analogues.

During this investigation, significant consideration was given to how such cell models would be developed. A useful first step would be towards the generation of a clonal subpopulation with stable integration of $\beta$ I-tubulin sequence containing the point mutation of interest. This would allow the establishment of clonal cell lines, with mutant $\beta I-t u b u l i n$ sequence present throughout the entire population. Following the generation of these cells, further genetic manipulations may be of use in the knockdown of wild type $\beta$-tubulin sequence utilising emerging biotechnologies capable of doing this in mammalian cells.

One particular approach for achieving gene silencing of the endogenous $\beta \mathrm{I}$-tubulin content within cells may be through the application of promoter targeted shRNA knockdown techniques. RNA mediated knockdown traditionally utilises an RNA sequence with antisense homology to a target RNA transcript of interest, which is introduced to cells via expression from a transfected plasmid, or by directly transfecting the appropriate nucleic acid sequence itself, thus mimicking the endogenous regulation mechanisms of non-coding RNAi (Hannon 2002, Meister and Tuschl 2004). These sequences form double stranded 
RNA molecules which are then targeted for degradation by Dicer and RISC associated regulatory mechanisms (for review see Tomari and Zamore (2005), Carthew and Sontheimer (2009)), leading to the degradation of RNA transcripts and suppression of a cells ability to produce the target protein of interest. In recent years, our growing understanding of the role of short non-coding RNA in epigenetic regulation has led to the development of a useful new tool for suppression of a target protein. It has been shown that shRNAs targeted to the promoter sequence of a target gene of interest can be utilised to induce long term epigenetic silencing in the target promoter element of mammalian cells. This occurs through the initiation of chromatin remodelling enzymes such as DNA methyltransferase Dnmt3A, Hystone deacetylase HDAC1, lysine methyltransferases KMT6 and KMT1C (for full review see Malecová and Morris (2010), Sibley, Seow et al. (2010), Roberts, Andaloussi et al. (2012)). This alternative approach, known as RNA mediated transcriptional gene silencing, was first reported in mammalian cells by Morris, Chan et al. (2004). Emergent applications in the silencing of highly active gene sequences such as demonstrated by the in vivo suppression of HIV-1 infection (Suzuki, Hattori et al. 2013), demonstrates the potent capabilities of this technique as it moves into mainstream applications among our available gene silencing techniques (Ross and Kassir 2014).

In the current study, traditional gene silencing by siRNA mediated knockdown is an inappropriate strategy as the RNA transcript of endogenous $\beta I$-tubulin sequence differs from our mutant $\beta$ I-tubulin variant of interest in only a single point mutation. By utilising a transcriptional gene silencing approach in cells with a stable integration of a $\beta I$-tubulin mutant sequence of interest, shRNA guide sequence with homology to a unique sequence within the $\beta$ I-tubulin promoter may be a useful mechanism through which to induce transcriptional gene silencing, as our mutant $\beta$ I-tubulin sequences are under the transcriptional regulation of a viral promoter sequence and thus able to avoid mechanisms 
of transcriptional gene silencing. This represents a potentially useful approach for increasing the resolution of concentration-response studies on individual $\beta$ I-tubulin point mutants in mammalian cells, dependent on the level of transcriptional gene silencing induced within the population. In addition, approaching the knockdown of endogenous $\beta I-$ tubulin sequence utilising transcriptional gene silencing techniques represents a potentially low cost strategy in comparison to current genome editing techniques such as zinc finger nucleases (ZFNs), transcription activator-like effector nucleases (TALENs) or clustered regularly interspaced short palindromic repeat targeting by CRISPR associated proteins (CRISPR/Cas).

Once developed, a method producing mutant $\beta I$-tubulin homodimers in high abundance would be advantageous for the verification of D297, or other interactions of interest, as contributors to stabilising $\beta$ I-tubulin interactions with Pel A and Lau. Expanding the investigation of D297 to additional cell lines in any immediate investigations would be highly valuable in generating further supporting evidence for the biological role of D297 in Pel A/Lau binding-associations. 


\section{References:}

Akhmanova, A. and M. O. Steinmetz (2008). "Tracking the ends: a dynamic protein network controls the fate of microtubule tips." Nat Rev Mol Cell Biol 9(4): 309-322.

Al-Lazikani, B., U. Banerji and P. Workman (2012). "Combinatorial drug therapy for cancer in the post-genomic era." Nat Biotech 30(7): 679-692.

Amos, L. A. and D. Schlieper (2005). Microtubules and Maps. Advances in Protein Chemistry. M. S. John and A. D. P. David, Academic Press. Volume 71: 257-298.

Andreu, J. M., J. Bordas, J. F. Diaz, J. Garcia de Ancos, R. Gil, F. J. Medrano, E. Nogales, E. Pantos and E. Towns-Andrews (1992). "Low resolution structure of microtubules in solution. Synchrotron X-ray scattering and electron microscopy of taxolinduced microtubules assembled from purified tubulin in comparison with glycerol and MAP-induced microtubules." J Mol Biol 226(1): 169-184.

Asthagiri, A. R., N. Pouratian, J. Sherman, G. Ahmed and M. E. Shaffrey (2007). "Advances in Brain Tumor Surgery." Neurologic Clinics 25(4): 975-1003.

Ballantyne, A. and S. Dhillon (2013). "Trastuzumab Emtansine: First Global Approval." Drugs 73(7): 755-765.

Begaye, A., S. Trostel, Z. Zhao, R. E. Taylor, D. C. Schriemer and D. L. Sackett (2011). "Mutations in the $\beta$-tubulin binding site for peloruside A confer resistance by targeting a cleft significant in side chain binding." Cell Cycle 10(19): 3387-3396.

Begaye, A., S. Trostel, Z. Zhao, R. E. Taylor, D. C. Schriemer and D. L. Sackett (2011). "Mutations in the $\beta$-tubulin binding site for peloruside A confer resistance by targeting a cleft significant in side chain binding." Cell Cycle 10(19): 3387.

Belarbi, E. H., A. Contreras Gómez, Y. Chisti, F. García Camacho and E. Molina Grima (2003). "Producing drugs from marine sponges." Biotechnology advances 21(7): 585-598.

Bennett, M. J., K. Barakat, J. T. Huzil, J. Tuszynski and D. C. Schriemer (2010). "Discovery and Characterization of the Laulimalide-Microtubule Binding Mode by Mass Shift Perturbation Mapping." Chemistry \&amp; Biology 17(7): 725-734.

Berridge, M. V., P. M. Herst and A. S. Tan (2005). "Tetrazolium dyes as tools in cell biology: new insights into their cellular reduction." Biotechnol Annu Rev 11: 127-152.

Bishop, C. L., M. Ramalho, N. Nadkarni, W. M. Kong, C. F. Higgins and N. Krauzewicz (2006). "Role for centromeric heterochromatin and PML nuclear bodies in the cellular response to foreign DNA." Molecular and cellular biology 26(7): 2583-2594. 
Black, P. M. and S. F. Ronner (1987). "Cortical mapping for defining the limits of tumor resection." Neurosurgery 20(6): 914-919.

Blackstock, W. P. and M. P. Weir (1999). "Proteomics: quantitative and physical mapping of cellular proteins." Trends in Biotechnology 17(3): 121-127.

Bollag, D. M., P. A. McQueney, J. Zhu, O. Hensens, L. Koupal, J. Liesch, M. Goetz, E. Lazarides and C. M. Woods (1995). "Epothilones, a new class of microtubule-stabilizing agents with a taxol-like mechanism of action." Cancer Res 55(11): 2325-2333.

Bolton, E. E., Y. Wang, P. A. Thiessen and S. H. Bryant (2008). "PubChem: integrated platform of small molecules and biological activities." Annual reports in computational chemistry 4: 217-241.

Borisy, G. G. and E. W. Taylor (1967). "Mechanism of action of colchicine - binding of colchicine-3H to cellular protein." Journal of Cell Biology 34(2): 525-\&.

Bos, P. D., X. H. F. Zhang, C. Nadal, W. Shu, R. R. Gomis, D. X. Nguyen, A. J. Minn, M. J. van de Vijver, W. L. Gerald, J. A. Foekens and J. Massague (2009). "Genes that mediate breast cancer metastasis to the brain." Nature 459(7249): 1005-1009.

Buey, R. M., E. Calvo, I. Barasoain, O. Pineda, M. C. Edler, R. Matesanz, G. Cerezo, C. D. Vanderwal, B. W. Day, E. J. Sorensen, J. A. Lopez, J. M. Andreu, E. Hamel and J. F. Diaz (2007). "Cyclostreptin binds covalently to microtubule pores and lumenal taxoid binding sites." Nat Chem Biol 3(2): 117-125.

Burkhart, C. A., M. Kavallaris and S. Band Horwitz (2001). "The role of beta-tubulin isotypes in resistance to antimitotic drugs." Biochim Biophys Acta 1471(2): O1-9.

Buss, A. D. and M. S. Butler (2004). "A new model for utilising chemical diversity from natural sources." Drug Development Research 62(4): 362-370.

Caplow, M., R. L. Ruhlen and J. Shanks (1994). "The free energy for hydrolysis of a microtubule-bound nucleotide triphosphate is near zero: all of the free energy for hydrolysis is stored in the microtubule lattice." J Cell Biol 127(3): 779-788.

Carthew, R. W. and E. J. Sontheimer (2009). "Origins and mechanisms of miRNAs and siRNAs." Cell 136(4): 642-655.

Chen, J. J., Y. L. Sun, A. K. Tiwari, Z. J. Xiao, K. Sodani, D. H. Yang, S. G. Vispute, W. Q. Jiang, S. D. Chen and Z. S. Chen (2012). "PDE5 inhibitors, sildenafil and vardenafil, reverse multidrug resistance by inhibiting the efflux function of multidrug resistance protein 7 (ATP - binding Cassette C10) transporter." Cancer science 103(8): 1531-1537.

Chesnoy, S. and L. Huang (2000). "Structure and function of lipid-DNA complexes for gene delivery." Annual review of biophysics and biomolecular structure 29(1): 27-47. 
Chrétien, D., F. Metoz, F. Verde, E. Karsenti and R. Wade (1992). "Lattice defects in microtubules: protofilament numbers vary within individual microtubules." The Journal of cell biology 117(5): 1031-1040.

Churchill, C. D., M. Klobukowski and J. A. Tuszynski (2014). "The Unique Binding Mode of Laulimalide to Two Tubulin Protofilaments." Chem Biol Drug Des.

Corley, D. G., R. Herb, R. E. Moore, P. J. Scheuer and V. J. Paul (1988). "Laulimalides. New potent cytotoxic macrolides from a marine sponge and a nudibranch predator." The Journal of Organic Chemistry 53(15): 3644-3646.

Cormier, A., M. Knossow, C. Wang and B. Gigant (2010). "The binding of vinca domain agents to tubulin: structural and biochemical studies." Methods Cell Biol 95: 373-390.

DeAngelis, L. M. (2001). "Brain tumors." N Engl J Med 344(2): 114-123.

Dhillon, S. (2014). "Trastuzumab Emtansine: A Review of Its Use in Patients with HER2-Positive Advanced Breast Cancer Previously Treated with Trastuzumab-Based Therapy." Drugs 74(6): 675-686.

Du Toit, A. (2014). "Cytoskeleton: Remodelling the FtsZ network." Nat Rev Mol Cell Biol 15(1): 3-3.

Dumontet, C., G. E. Durán, K. A. Steger, G. L. Murphy, H. H. Sussman and B. I. Sikic (1996). "Differential expression of tubulin isotypes during the cell cycle." Cell Motility and the Cytoskeleton 35(1): 49-58.

Dumontet, C. and M. Jordan (2010). "Microtubule-binding agents: a dynamic field of cancer therapeutics." Nat Rev Drug Discov 9(10): 790-803.

Dumontet, C. and M. A. Jordan (2010). "Microtubule-binding agents: a dynamic field of cancer therapeutics." Nature reviews Drug discovery 9(10): 790-803.

Dumontet, C., M. A. Jordan and F. F. Lee (2009). "Ixabepilone: targeting $\beta I I I-t u b u l i n$ expression in taxane-resistant malignancies." Molecular cancer therapeutics 8(1): 17-25.

Dutcher, S. K. (2001). "The tubulin fraternity: alpha to eta." Current Opinion in Cell Biology 13(1): 49-54.

Elie-Caille, C., F. Severin, J. Helenius, J. Howard, D. J. Muller and A. A. Hyman (2007). "Straight GDP-tubulin protofilaments form in the presence of taxol." Curr Biol 17(20): 1765-1770.

Erickson, H. P. (1995). "FtsZ, a prokaryotic homolog of tubulin?" Cell 80(3): 367-370.

Erickson, H. P. (2007). "Evolution of the cytoskeleton." Bioessays 29(7): 668-677. 
Evan E. Bolton, Y. W., Paul A. Thiessen, Stephen H. Bryant*. (2014). "PubChem: Integrated Platform of Small Molecules and Biological Activities." from http://www.ncbi.nlm.nih.gov/pccompound.

Evans, W. E. and H. L. McLeod (2003). "Pharmacogenomics - Drug Disposition, Drug Targets, and Side Effects." New England Journal of Medicine 348(6): 538-549.

Faulkner, D. J. (2002). "Marine natural products." Natural Product Reports 19(1): 1-48.

Field, C., R. Li and K. Oegema (1999). "Cytokinesis in eukaryotes: a mechanistic comparison." Current Opinion in Cell Biology 11(1): 68-80.

Field, Jessica J., José F. Díaz and John H. Miller "The Binding Sites of MicrotubuleStabilizing Agents." Chemistry \& Biology 20(3): 301-315.

Field, J. J., A. Kanakkanthara and J. H. Miller (2014). "Microtubule-targeting agents are clinically successful due to both mitotic and interphase impairment of microtubule function." Bioorganic \& Medicinal Chemistry 22(18): 5050-5059.

Field, J. J., B. Pera, E. Calvo, A. Canales, D. Zurwerra, C. Trigili, J. Rodríguez-Salarichs, R. Matesanz, A. Kanakkanthara, S. J. Wakefield, A. J. Singh, J. Jiménez-Barbero, P. Northcote, J. H. Miller, J. A. López, E. Hamel, I. Barasoain, K.-H. Altmann and J. F. Díaz (2012). "Zampanolide, a potent new microtubule stabilizing agent, covalently reacts with the taxane luminal site in both tubulin $\alpha, \beta$-heterodimers and microtubules." Chemistry \& biology 19(6): 686-698.

Futreal, P. A., L. Coin, M. Marshall, T. Down, T. Hubbard, R. Wooster, N. Rahman and M. R. Stratton (2004). "A census of human cancer genes." Nature Reviews Cancer 4(3): 177-183.

Gaitanos, T., R. Buey, F. Díaz, P. Northcote, P. Teesdale-Spittle, J. Andreu and J. Miller (2004). "Peloruside A Does Not Bind to the Taxoid Site on $\beta$-Tubulin and Retains Its Activity in Multidrug-Resistant Cell Lines." Cancer Research 64(15): 5063-5067.

Gaitanos, T. N., R. M. Buey, J. F. Díaz, P. T. Northcote, P. Teesdale-Spittle, J. M. Andreu and J. H. Miller (2004). "Peloruside A does not bind to the taxoid site on $\beta$ tubulin and retains its activity in multidrug-resistant cell lines." Cancer research 64(15): 5063-5067.

García-Mata, R., Z. Bebök, E. J. Sorscher and E. S. Sztul (1999). "Characterization and Dynamics of Aggresome Formation by a Cytosolic Gfp-Chimera." The Journal of cell biology 146(6): 1239-1254.

Gelderblom, H., J. Verweij, K. Nooter and A. Sparreboom (2001). "Cremophor EL: the drawbacks and advantages of vehicle selection for drug formulation." Eur J Cancer 37(13): 1590-1598. 
Gerth, K., N. Bedorf, G. Hofle, H. Irschik and H. Reichenbach (1996). "Epothilons A and B: antifungal and cytotoxic compounds from Sorangium cellulosum (Myxobacteria). Production, physico-chemical and biological properties." J Antibiot (Tokyo) 49(6): 560563.

Giannakakou, P., D. L. Sackett, Y.-K. Kang, Z. Zhan, J. T. Buters, T. Fojo and M. S. Poruchynsky (1997). "Paclitaxel-resistant human ovarian cancer cells have mutant $\beta$ tubulins that exhibit impaired paclitaxel-driven polymerization." Journal of Biological Chemistry 272(27): 17118-17125.

Gonzalez-Garay, M. L. and F. Cabral (1995). "Overexpression of an epitope- tagged $\beta$ tubulin in Chinese hamster ovary cells causes an increase in endogenous $\alpha$-tubulin synthesis." Cell Motility and the Cytoskeleton 31(4): 259-272.

Gottesman, M. M. (2002). "Mechanisms of cancer drug resistance." Annu Rev Med 53: 615-627.

Hamel, E., B. Day, J. Miller, K. Jung, P. Northcote, A. Ghosh, D. Curran, M. Cushman, K. C. Nicolaou, I. Paterson and E. Sorensen (2006). "Synergistic Effects of Peloruside A and Laulimalide with Taxoid Site Drugs, but Not with Each Other, on Tubulin Assembly." Molecular Pharmacology 70(5): 1555-1564.

Han, D. K., J. Eng, H. Zhou and R. Aebersold (2001). "Quantitative profiling of differentiation-induced microsomal proteins using isotope-coded affinity tags and mass spectrometry." Nat Biotechnol 19(10): 946-951.

Hanahan, D. and R. A. Weinberg (2000). "The hallmarks of cancer." cell 100(1): 57-70.

Hanna, R., D. R. Maass, P. H. Atkinson, P. T. Northcote, P. H. Teesdale-Spittle, D. S. Bellows and J. H. Miller (2014). "Characterizing the laulimalide-peloruside binding site using site-directed mutagenesis of TUB2 in S. cerevisiae." Mol Biosyst 10(1): 110-116.

Hannon, G. J. (2002). "RNA interference." Nature 418(6894): 244-251.

Hari, M., F. Loganzo, T. Annable, X. Tan, S. Musto, D. B. Morilla, J. H. Nettles, J. P. Snyder and L. M. Greenberger (2006). "Paclitaxel-resistant cells have a mutation in the paclitaxel-binding region of $\beta$-tubulin (Asp26Glu) and less stable microtubules." Molecular cancer therapeutics 5(2): 270-278.

Harvey, A. L., R. L. Clark, S. P. Mackay and B. F. Johnston (2010). "Current strategies for drug discovery through natural products." Expert opinion on drug discovery 5(6): 559568 .

Hasse, A., W. A. Schulz and H. Sies (1992). "De novo methylation of transfected CAT gene plasmid constructs in F9 mouse embryonal carcinoma cells." Biochimica et Biophysica Acta (BBA)-Gene Structure and Expression 1131(1): 16-22. 
Hayden, J. H., S. S. Bowser and C. L. Rieder (1990). "Kinetochores capture astral microtubules during chromosome attachment to the mitotic spindle: direct visualization in live newt lung cells." The Journal of cell biology 111(3): 1039-1045.

Hessling, B., K. Buttner, M. Hecker and D. Becher (2013). "Global relative quantification with liquid chromatography-matrix-assisted laser desorption ionization time-of-flight (LC-MALDI-TOF)--cross-validation with LTQ-Orbitrap proves reliability and reveals complementary ionization preferences." Mol Cell Proteomics 12(10): 2911-2920.

Hood, J. D. and D. A. Cheresh (2002). "Role of integrins in cell invasion and migration." Nat Rev Cancer 2(2): 91-100.

Hood, K. A., B. T. Bäckström, P. T. Northcote and M. V. Berridge (2001). "The novel cytotoxic sponge metabolite peloruside A, structurally similar to bryostatin - 1 , has unique bioactivity independent of protein kinase C." Anti-cancer drug design 16(2): 155166.

Hood, K. A., L. M. West, B. Rouwé, P. T. Northcote, M. V. Berridge and J. H. Miller (2002). "Peloruside A, a novel antimitotic agent with paclitaxel-like microtubulestabilizing activity." Cancer research 62(12): 3356-3360.

Hopper-Borge, E., Z.-S. Chen, I. Shchaveleva, M. G. Belinsky and G. D. Kruh (2004). "Analysis of the Drug Resistance Profile of Multidrug Resistance Protein 7 (ABCC10) Resistance to Docetaxel." Cancer research 64(14): 4927-4930.

Huzil, J. T., K. Chen, L. Kurgan and J. A. Tuszynski (2007). "The Roles of $\beta$-Tubulin Mutations and Isotype Expression in Acquired Drug Resistance." Cancer Informatics 3: 159-181.

Huzil, J. T., J. K. Chik, G. W. Slysz, H. Freedman, J. Tuszynski, R. E. Taylor, D. L. Sackett and D. C. Schriemer (2008). "A unique mode of microtubule stabilization induced by peloruside A." Journal of molecular biology 378(5): 1016-1030.

Huzil, T., J. Chik, G. Slysz, H. Freedman, J. Tuszynski, R. Taylor, D. Sackett and D. Schriemer (2008). "A Unique Mode of Microtubule Stabilization Induced by Peloruside A." Journal of Molecular Biology 378(5): 1016-1030.

Janke, C., M. M. Magiera, N. Rathfelder, C. Taxis, S. Reber, H. Maekawa, A. MorenoBorchart, G. Doenges, E. Schwob, E. Schiebel and M. Knop (2004). "A versatile toolbox for PCR-based tagging of yeast genes: new fluorescent proteins, more markers and promoter substitution cassettes." Yeast 21(11): 947-962.

Janosi, I. M., D. Chretien and H. Flyvbjerg (2002). "Structural microtubule cap: stability, catastrophe, rescue, and third state." Biophys J 83(3): 1317-1330.

Jemal, A., F. Bray, M. M. Center, J. Ferlay, E. Ward and D. Forman (2011). "Global cancer statistics." CA Cancer J Clin 61(2): 69-90. 
Johnson, T. A., K. Tenney, R. H. Cichewicz, B. I. Morinaka, K. N. White, T. Amagata, B. Subramanian, J. Media, S. L. Mooberry, F. A. Valeriote and P. Crews (2007). "Spongederived fijianolide polyketide class: further evaluation of their structural and cytotoxicity properties." J Med Chem 50(16): 3795-3803.

Jordan, M. and L. Wilson (2004). "Microtubules as a target for anticancer drugs." Nat Rev Cancer 4(4): 253-265.

Jordan, M. A., K. Wendell, S. Gardiner, W. B. Derry, H. Copp and L. Wilson (1996). "Mitotic block induced in HeLa cells by low concentrations of paclitaxel (Taxol) results in abnormal mitotic exit and apoptotic cell death." Cancer research 56(4): 816-825.

Jordan, M. A. and L. Wilson (2004). "Microtubules as a target for anticancer drugs." Nat Rev Cancer 4(4): 253-265.

Kaelin, W. (2005). "The Concept of Synthetic Lethality in the Context of Anticancer Therapy." Nat Rev Cancer 5(9): 689-698.

Kanakkanthara, A., J. Eras, P. T Northcote, F. Cabral and J. H Miller (2014). "Resistance to Peloruside A and Laulimalide: Functional Significance of Acquired $\beta I$-tubulin Mutations at Sites Important for Drug-Tubulin Binding." Current cancer drug targets 14(1): 79-90.

Kanakkanthara, A., A. Wilmes, A. O'Brate, D. Escuin, A. Chan, A. Gjyrezi, J. Crawford, P. Rawson, B. Kivell and P. T. Northcote (2011). "Peloruside-and laulimalide-resistant human ovarian carcinoma cells have $\beta$ I-tubulin mutations and altered expression of $\beta$ IIand $\beta$ III-tubulin isotypes." Molecular cancer therapeutics 10(8): 1419-1429.

Kaplan, R. N., R. D. Riba, S. Zacharoulis, A. H. Bramley, L. Vincent, C. Costa, D. D. MacDonald, D. K. Jin, K. Shido, S. A. Kerns, Z. P. Zhu, D. Hicklin, Y. Wu, J. L. Port, N. Altorki, E. R. Port, D. Ruggero, S. V. Shmelkov, K. K. Jensen, S. Rafii and D. Lyden (2005). "VEGFR1-positive haematopoietic bone marrow progenitors initiate the premetastatic niche." Nature 438(7069): 820-827.

Kathawala, R. J., L. Wei, N. Anreddy, K. Chen, A. Patel, S. Alqahtani, Y.-K. Zhang, Y.J. Wang, K. Sodani and A. Kaddoumi (2014). "The small molecule tyrosine kinase inhibitor NVP-BHG712 antagonizes ABCC10-mediated paclitaxel resistance: a preclinical and pharmacokinetic study." Oncotarget.

Kavallaris, M. (2010). "Microtubules and resistance to tubulin-binding agents." Nat Rev Cancer 10(3): 194-204.

Kelling, J., K. Sullivan, L. Wilson and M. A. Jordan (2003). "Suppression of centromere dynamics by Taxol in living osteosarcoma cells." Cancer Res 63(11): 2794-2801.

Khrapunovich-Baine, M., V. Menon, C.-P. H. Yang, P. T. Northcote, J. H. Miller, R. H. Angeletti, A. Fiser, S. B. Horwitz and H. Xiao (2011). "Hallmarks of molecular action of 
microtubule stabilizing agents effects of epothilone $\mathrm{B}$, ixabepilone, peloruside $\mathrm{A}$, and laulimalide on microtubule conformation." Journal of Biological Chemistry 286(13): 11765-11778.

Komlodi-Pasztor, E., D. Sackett, J. Wilkerson and T. Fojo (2011). "Mitosis is not a key target of microtubule agents in patient tumors." Nat Rev Clin Oncol 8(4): 244-250.

Komlodi-Pasztor, E., D. L. Sackett and A. T. Fojo (2012). "Inhibitors targeting mitosis: tales of how great drugs against a promising target were brought down by a flawed rationale." Clin Cancer Res 18(1): 51-63.

Larocque, K., P. Ovadje, S. Djurdjevic, M. Mehdi, J. Green and S. Pandey (2014). "Novel Analogue of Colchicine Induces Selective Pro-Death Autophagy and Necrosis in Human Cancer Cells." PLoS ONE 9(1): e87064.

Larsen, E. M., M. R. Wilson, J. Zajicek and R. E. Taylor (2013). "Conformational Preferences of Zampanolide and Dactylolide." Organic letters 15(20): 5246-5249.

Ledbetter, M. C. and K. R. Porter (1963). "A "microtubule" in plant cell fine structure." J Cell Biol 19(1): 239-250.

Lewis, S. A., G. Tian, I. E. Vainberg and N. J. Cowan (1996). "Chaperonin-mediated folding of actin and tubulin." J Cell Biol 132(1-2): 1-4.

Li, H., D. J. DeRosier, W. V. Nicholson, E. Nogales and K. H. Downing (2002). "Microtubule Structure at 8 A Resolution." Structure 10(10): 1317-1328.

Li, J., A. L. Risinger and S. L. Mooberry (2014). "Taccalonolide microtubule stabilizers." Bioorg Med Chem 22(18): 5091-5096.

Li, J., A. L. Risinger, J. Peng, Z. Chen, L. Hu and S. L. Mooberry (2011). "Potent taccalonolides, AF and AJ, inform significant structure-activity relationships and tubulin as the binding site of these microtubule stabilizers." J Am Chem Soc 133(47): 1906419067.

Liang, P. and T. H. MacRae (1997). "Molecular chaperones and the cytoskeleton." Journal of Cell Science 110: 1431-1440.

Liu, J., M. Towle, H. Cheng, P. Saxton, C. Reardon, J. Wu, E. Murphy, G. Kuznetsov, C. Johannes, M. Tremblay, H. Zhao, M. Pesant, F. Fang, M. Vermeulen, Brian and B. Littlefield (2007). "In Vitro and In Vivo Anticancer Activities of Synthetic (-)Laulimalide, a Marine Natural Product Microtubule Stabilizing Agent." Anticancer Research 27(3B): 1509-1518.

Liu, J., M. J. Towle, H. Cheng, P. Saxton, C. Reardon, J. Wu, E. A. Murphy, G. Kuznetsov, C. W. Johannes, M. R. Tremblay, H. Zhao, M. Pesant, F. G. Fang, M. W. 
Vermeulen, B. M. Gallagher, Jr. and B. A. Littlefield (2007). "In vitro and in vivo anticancer activities of synthetic (-)-laulimalide, a marine natural product microtubule stabilizing agent." Anticancer Res 27(3b): 1509-1518.

Leach, A. R. and M. M. Hann (2000). "The in silico world of virtual libraries." Drug Discov Today 5(8): 326-336.

Lowe, J., H. Li, K. H. Downing and E. Nogales (2001). "Refined structure of alpha betatubulin at 3.5 A resolution." J Mol Biol 313(5): 1045-1057.

Luduena, R. F. (1998). "Multiple forms of tubulin: different gene products and covalent modifications." Int Rev Cytol 178: 207-275.

Ludueña, R. F. (1993). "Are tubulin isotypes functionally significant." Molecular Biology of the Cell 4(5): 445-457.

Malecová, B. and K. V. Morris (2010). "Transcriptional gene silencing mediated by noncoding RNAs." Current opinion in molecular therapeutics 12(2): 214-222.

Massarotti, A., A. Coluccia, R. Silvestri, G. Sorba and A. Brancale (2012). "The tubulin colchicine domain: a molecular modeling perspective." ChemMedChem 7(1): 33-42.

Matesanz, R., J. Rodriguez-Salarichs, B. Pera, A. Canales, J. M. Andreu, J. JimenezBarbero, W. Bras, A. Nogales, W. S. Fang and J. F. Diaz (2011). "Modulation of microtubule interprotofilament interactions by modified taxanes." Biophys J 101(12): 2970-2980.

Meister, G. and T. Tuschl (2004). "Mechanisms of gene silencing by double-stranded RNA." Nature 431(7006): 343-349.

Mitchison, T. and M. Kirschner (1984). "Dynamic instability of microtubule growth." nature 312(5991): 237-242.

Miyamoto, D. T., Z. E. Perlman, T. J. Mitchison and M. Shirasu-Hiza (2003). "Dynamics of the mitotic spindle--potential therapeutic targets." Progress in cell cycle research 5: 349-360.

Mooberry, S., G. Tien, A. Hernandez, A. Plubrukarn and B. Davidson (1999). "Laulimalide and Isolaulimalide, New Paclitaxel-Like Microtubule-Stabilizing Agents." Cancer Research 59(3): 653-660.

Morris, K. V., S. W. Chan, S. E. Jacobsen and D. J. Looney (2004). "Small interfering RNA-induced transcriptional gene silencing in human cells." Science 305(5688): 12891292. 
Mosmann, T. (1983). "Rapid colorimetric assay for cellular growth and survival: application to proliferation and cytotoxicity assays." Journal of immunological methods 65(1): 55-63.

Müller, A., B. Homey, H. Soto, N. Ge, D. Catron, M. E. Buchanan, T. McClanahan, E. Murphy, W. Yuan and S. N. Wagner (2001). "Involvement of chemokine receptors in breast cancer metastasis." nature 410(6824): 50-56.

Newman, D. J. and G. M. Cragg (2012). "Natural Products As Sources of New Drugs over the 30 Years from 1981 to 2010." Journal of Natural Products 75(3): 311-335.

Nguyen, T. L., X. Xu, R. Gussio, A. K. Ghosh and E. Hamel (2010). "The assemblyinducing laulimalide/peloruside A binding site on tubulin: molecular modeling and biochemical studies with $[3 \mathrm{H}]$ peloruside A." Journal of chemical information and modeling 50(11): 2019-2028.

Nogales, E. (2000). "Structural insights into microtubule function." Annu Rev Biochem 69: $277-302$.

Nogales, E., H. W. Wang and H. Niederstrasser (2003). "Tubulin rings: which way do they curve?" Curr Opin Struct Biol 13(2): 256-261.

Nogales, E., M. Whittaker, R. A. Milligan and K. H. Downing (1999). "High-resolution model of the microtubule." Cell 96(1): 79-88.

Panowski, S., S. Bhakta, H. Raab, P. Polakis and J. R. Junutula (2014). Site-specific antibody drug conjugates for cancer therapy. MAbs, Landes Bioscience.

Peng, J., A. L. Risinger, G. A. Fest, E. M. Jackson, G. Helms, L. A. Polin and S. L. Mooberry (2011). "Identification and Biological Activities of New Taccalonolide Microtubule Stabilizers." Journal of medicinal chemistry 54(17): 6117-6124.

Pointer, K. B., P. A. Clark, M. Zorniak, B. M. Alrfaei and J. S. Kuo (2014). "Glioblastoma cancer stem cells: Biomarker and therapeutic advances." Neurochemistry International 71(0): 1-7.

Prota, A. E., K. Bargsten, P. T. Northcote, M. Marsh, K. H. Altmann, J. H. Miller, J. F. Díaz and M. O. Steinmetz (2014). "Structural basis of microtubule stabilization by laulimalide and peloruside A." Angewandte Chemie 126(6): 1647-1651.

Prota, A. E., K. Bargsten, D. Zurwerra, J. J. Field, J. F. Díaz, K.-H. Altmann and M. O. Steinmetz (2013). "Molecular mechanism of action of microtubule-stabilizing anticancer agents." Science 339(6119): 587-590. 
Prota, A. E., M. M. Magiera, M. Kuijpers, K. Bargsten, D. Frey, M. Wieser, R. Jaussi, C. C. Hoogenraad, R. A. Kammerer and C. Janke (2013). "Structural basis of tubulin tyrosination by tubulin tyrosine ligase." The Journal of cell biology 200(3): 259-270.

Pryor, D. E., A. O'Brate, G. Bilcer, J. F. Díaz, Y. Wang, Y. Wang, M. Kabaki, M. K. Jung, J. M. Andreu, A. K. Ghosh, P. Giannakakou and E. Hamel (2002). "The Microtubule Stabilizing Agent Laulimalide Does Not Bind in the Taxoid Site, Kills Cells Resistant to Paclitaxel and Epothilones, and May Not Require Its Epoxide Moiety for Activity." Biochemistry 41(29): 9109-9115.

Qiu, G.-H., C. H.-W. Leung, T. Yun, X. Xie, M. Laban and S. C. Hooi (2011). "Recognition and Suppression of Transfected Plasmids by Protein ZNF511-PRAP1, a Potential Molecular Barrier to Transgene Expression." Mol Ther 19(8): 1478-1486.

Quinoa, E., Y. Kakou and P. Crews (1988). "Fijianolides, polyketide heterocycles from a marine sponge." The Journal of Organic Chemistry 53(15): 3642-3644.

Ravelli, R. B., B. Gigant, P. A. Curmi, I. Jourdain, S. Lachkar, A. Sobel and M. Knossow (2004). "Insight into tubulin regulation from a complex with colchicine and a stathminlike domain." Nature 428(6979): 198-202.

Risinger, A. L., C. D. Westbrook, A. Encinas, M. Mülbaier, C. M. Schultes, S. Wawro, J. D. Lewis, B. Janssen, F. J. Giles and S. L. Mooberry (2011). "ELR510444, A Novel Microtubule Disruptor with Multiple Mechanisms of Action." The Journal of Pharmacology and Experimental Therapeutics 336(3): 652-660.

Riu, E., Z.-Y. Chen, H. Xu, C.-Y. He and M. A. Kay (2007). "Histone modifications are associated with the persistence or silencing of vector-mediated transgene expression in vivo." Molecular Therapy 15(7): 1348-1355.

Roberts, T. C., S. E. Andaloussi, K. V. Morris, G. McClorey and M. J. Wood (2012). "Small RNA-Mediated Epigenetic Myostatin Silencing." Mol Ther Nucleic Acids 1: e23.

Rohena, C. C. and S. L. Mooberry (2014). "Recent progress with microtubule stabilizers: new compounds, binding modes and cellular activities." Natural product reports 31(3): $335-355$

Rohena, C. C. and S. L. Mooberry (2014). "Recent progress with microtubule stabilizers: new compounds, binding modes and cellular activities." Nat Prod Rep 31(3): 335-355.

Ross, J. P. and Z. Kassir (2014). "The Varied Roles of Nuclear Argonaute-Small RNA Complexes and Avenues for Therapy." Molecular Therapy. Nucleic Acids 3(10): e203.

Rusan, N. M., C. J. Fagerstrom, A. M. Yvon and P. Wadsworth (2001). "Cell cycledependent changes in microtubule dynamics in living cells expressing green fluorescent protein-alpha tubulin." Mol Biol Cell 12(4): 971-980. 
Schiff, P. B., J. Fant and S. B. Horwitz (1979). "Promotion of microtubule assembly in vitro by taxol." Nature 277(5698): 665-667.

Seddon, G., V. Lounnas, R. McGuire, T. van den Bergh, R. P. Bywater, L. Oliveira and G. Vriend (2012). "Drug design for ever, from hype to hope." Journal of computer-aided molecular design 26(1): 137-150.

Sheinerman, F. B., R. Norel and B. Honig (2000). "Electrostatic aspects of proteinprotein interactions." Current Opinion in Structural Biology 10(2): 153-159.

Shen, H., F. Y. Lee and J. Gan (2011). "Ixabepilone, a novel microtubule-targeting agent for breast cancer, is a substrate for P-glycoprotein (P-gp/MDR1/ABCB1) but not breast cancer resistance protein (BCRP/ABCG2)." J Pharmacol Exp Ther 337(2): 423-432.

Sibley, C. R., Y. Seow and M. J. Wood (2010). "Novel RNA-based strategies for therapeutic gene silencing." Mol Ther 18(3): 466-476.

Siegel, R., D. Naishadham and A. Jemal (2012). "Cancer statistics, 2012." CA: A Cancer Journal for Clinicians 62(1): 10-29.

Sipkema, D., M. Franssen, R. Osinga, J. Tramper and R. Wijffels (2005). "Marine Sponges as Pharmacy." Marine Biotechnology 7(3): 142-162.

Slautterback, D. B. (1963). "Cytoplasmic microtubules. I. Hydra." J Cell Biol 18: 367388.

Stratton, M. R., P. J. Campbell and P. A. Futreal (2009). "The cancer genome." Nature 458(7239): 719-724.

Stupp, R., W. P. Mason, M. J. van den Bent, M. Weller, B. Fisher, M. J. Taphoorn, K. Belanger, A. A. Brandes, C. Marosi, U. Bogdahn, J. Curschmann, R. C. Janzer, S. K. Ludwin, T. Gorlia, A. Allgeier, D. Lacombe, J. G. Cairncross, E. Eisenhauer and R. O. Mirimanoff (2005). "Radiotherapy plus concomitant and adjuvant temozolomide for glioblastoma." N Engl J Med 352(10): 987-996.

Sullivan, K. F. (1988). "Structure and utilization of tubulin isotypes." Annual review of cell biology 4(1): 687-716.

Suzuki, K., S. Hattori, K. Marks, C. Ahlenstiel, Y. Maeda, T. Ishida, M. Millington, M. Boyd, G. Symonds, D. A. Cooper, S. Okada and A. D. Kelleher (2013). "Promoter Targeting shRNA Suppresses HIV-1 Infection In vivo Through Transcriptional Gene Silencing." Molecular Therapy. Nucleic Acids 2(12): e137.

Taylor, S. and J. M. Peters (2008). "Polo and Aurora kinases; lessons derived from chemical biology." Current Opinion in Cell Biology 20(1): 77-84. 
Thakur, N. L. and W. E. Müller (2004). "Biotechnological potential of marine sponges." Curr Sci 86(11): 1506-1512.

Thepchatri, P., D. O. Cicero, E. Monteagudo, A. K. Ghosh, B. Cornett, E. R. Weeks and J. P. Snyder (2005). "Conformations of Laulimalide in DMSO-d6." Journal of the American Chemical Society 127(37): 12838-12846.

Tian, G. L., Y. Huang, H. Rommelaere, J. Vandekerckhove, C. Ampe and N. J. Cowan (1996). "Pathway leading to correctly folded beta-tubulin." Cell 86(2): 287-296.

Tinley, T. L., D. A. Randall-Hlubek, R. M. Leal, E. M. Jackson, J. W. Cessac, J. C. Quada, Jr., T. K. Hemscheidt and S. L. Mooberry (2003). "Taccalonolides E and A: Plant-derived steroids with microtubule-stabilizing activity." Cancer Res 63(12): 32113220 .

Tomari, Y. and P. D. Zamore (2005). "Perspective: machines for RNAi." Genes Dev 19(5): 517-529.

Verdier-Pinard, P., E. Pasquier, H. Xiao, B. Burd, C. Villard, D. Lafitte, L. M. Miller, R. H. Angeletti, S. B. Horwitz and D. Braguer (2009). "Tubulin proteomics: towards breaking the code." Anal Biochem 384(2): 197-206.

Wang, Y., G. Tian, N. J. Cowan and F. Cabral (2006). "Mutations affecting $\beta$-tubulin folding and degradation." Journal of Biological Chemistry 281(19): 13628-13635.

Wang, Y., S. Yin, K. Blade, G. Cooper, D. R. Menick and F. Cabral (2006). "Mutations at Leucine 215 of $\beta$-Tubulin Affect Paclitaxel Sensitivity by Two Distinct Mechanisms $\dagger$." Biochemistry 45(1): 185-194.

Wani, M. C., H. L. Taylor, M. E. Wall, P. Coggon and A. T. McPhail (1971). "Plant antitumor agents. VI. The isolation and structure of taxol, a novel antileukemic and antitumor agent from Taxus brevifolia." J Am Chem Soc 93(9): 2325-2327.

Weisenberg, R. C., G. G. Borisy and E. W. Taylor (1968). "Colchicine-binding protein of mammalian brain and its relation to microtubules." Biochemistry 7(12): 4466-4479.

West, L. M., P. T. Northcote and C. N. Battershill (2000). "Peloruside A: a potent cytotoxic macrolide isolated from the new zealand marine sponge Mycale sp." The Journal of organic chemistry $\mathbf{6 5}(2)$ : 445-449.

Wickstead, B. and K. Gull (2011). "The evolution of the cytoskeleton." The Journal of cell biology 194(4): 513-525.

Wilmes, A., K. Bargh, C. Kelly, P. T. Northcote and J. H. Miller (2007). "Peloruside A Synergizes with Other Microtubule Stabilizing Agents in Cultured Cancer Cell Lines." Molecular Pharmaceutics 4(2): 269-280. 
Witherup, K. M., S. A. Look, M. W. Stasko, T. J. Ghiorzi, G. M. Muschik and G. M. Cragg (1990). "Taxus Spp. needles contain ammounts of Taxol comparable to the bark of Taxus-Brevifolia - Analysis and isolation." Journal of Natural Products 53(5): 1249-1255.

Wittmann, T., A. Hyman and A. Desai (2001). "The spindle: a dynamic assembly of microtubules and motors." Nat Cell Biol 3(1): E28-E34.

Yang, H., A. Ganguly and F. Cabral (2010). "Inhibition of cell migration and cell division correlates with distinct effects of microtubule inhibiting drugs." Journal of Biological Chemistry 285(42): 32242-32250.

Younes, A., U. Yasothan and P. Kirkpatrick (2012). "Brentuximab vedotin." Nat Rev Drug Discov 11(1): 19-20.

Yue, Q.-X., X. Liu and D.-A. Guo (2010). "Microtubule-binding natural products for cancer therapy." Planta medica 76(11): 1037-1043.

Yue, Q. X., X. Liu and D. A. Guo (2010). "Microtubule-binding natural products for cancer therapy." Planta Med 76(11): 1037-1043. 


\section{Appendices}

\section{Appendix A: General Recipes}

Acrylamide Gels for SDS-PAGE:

$10 \%$ resolving gel - preparation for two gels

Milli-Q dd $\mathrm{H}_{2} \mathrm{O} \quad 8.0 \mathrm{~mL}$

1.5 M Tris (pH to 8.8 by $\mathrm{HCl}) \quad 5.0 \mathrm{~mL}$

$10 \%$ SDS $200.0 \mu \mathrm{L}$

$30 \%$ bis-acrylamide $\quad 6.66 \mathrm{~mL}$

$10 \%$ APS $\quad 100.0 \mu \mathrm{L}$

$\begin{array}{ll}\text { TEMED } & 10.0 \mu \mathrm{L}\end{array}$

(Overlay gel with $100 \%$ isopropanol while setting)

$4 \%$ Stacking gel - preparation for two gels

\begin{tabular}{ll} 
Milli-Q ddH & $\mathrm{O}$ \\
\hline
\end{tabular}

1.5 M Tris (pH to 6.8 with conc. $\mathrm{HCl}$ ) $2.5 \mathrm{~mL}$

$10 \%$ SDS $100.0 \mu \mathrm{L}$

$30 \%$ bis-acrylamide $\quad 1.33 \mathrm{~mL}$

$10 \%$ APS $\quad 50.0 \mu \mathrm{L}$

$\begin{array}{ll}\text { TEMED } & 10.0 \mu \mathrm{L}\end{array}$

DNA Separation Gels - Preparation of single gel

Agarose gel (1\%)

Agarose

$35.0 \mathrm{mg}$

$1 \times \mathrm{TBE}$

$35.0 \mathrm{~mL}$ 
Cell lysis buffers

RIPA buffer (100 mL)

1M Tris-HCL (pH 7.6) 2.5 mL

$\mathrm{NaCl}$

$0.88 \mathrm{~g}$

EDTA

$0.15 \mathrm{~g}$

SDS

$0.1 \mathrm{~g}$

Triton X-100

$1.0 \mathrm{~mL}$

Sodium deoxycholate

$1.0 \mathrm{~g}$

(pH to 7.5)

Dilute to $100 \mathrm{~mL}$ with Milli-Q ddH $\mathrm{H}_{2} \mathrm{O}$

Hypotonic lysis buffer (1 L)

$1.0 \mathrm{mM} \mathrm{MgCl} 2$

$95.211 \mathrm{mg}$

$2.0 \mathrm{mM}$ EGTA,

$0.7607 \mathrm{~g}$

$1 \%$ Nonidet P-40

$1.0 \mathrm{~mL}$

$50 \mathrm{mM}$ Tris- $\mathrm{HCl}$ $6.057 \mathrm{~g}$

$10 \mathrm{uL} / \mathrm{mL}$ protease inhibitor cocktail prior to lysis

Dilute to $100 \mathrm{~mL}$ with Milli-Q ddH $\mathrm{H}_{2} \mathrm{O}$

Colloidal Coomassie fixative $(500 \mathrm{~mL})$

$50 \%$ Ethanol

$250.0 \mathrm{~mL}$

$3.0 \%$ Ortho-phosphoric acid

$15.0 \mathrm{~mL}$

Dilute to $500 \mathrm{~mL}$ with Milli-Q ddH $\mathrm{H}_{2} \mathrm{O}$

Colloidal Coomassie staining solution $(500 \mathrm{~mL})$

Coomassie brilliant blue G250

$3.0 \mathrm{mg}$

$10 \%$ Acetic acid

$50.0 \mathrm{~mL}$

$34 \%$ Methanol

$170.0 \mathrm{~mL}$ 
Dilute to $100 \mathrm{~mL}$ with Milli-Q ddH $\mathrm{H}_{2} \mathrm{O}$

MTT Solution (50 mL)

3-(4,5-dimethylthiazol-2-yl)-2,5-diphenyltetrazolium bromide)

$250.0 \mathrm{mg}$

$1 \mathrm{x}$ PBS to $50 \mathrm{~mL}$

Sterile filter and protect from light exposure. Kept sterile

MTT solubilizer $(500 \mathrm{~mL})$

$10 \% \operatorname{SDS}$

$50.0 \mathrm{~g}$

$45 \% \mathrm{~N}, \mathrm{~N}-\mathrm{DMF}$

$225.0 \mathrm{~mL}$

Dilute to $500 \mathrm{~mL}$ with Milli-Q ddH $\mathrm{H}_{2} \mathrm{O}$

$\mathrm{pH}$ adjusted to 4.5 using glacial acetic acid

$10 \times$ PBS (1L)

$1.37 \mathrm{M} \mathrm{NaCL}$

$80.0 \mathrm{~g}$

$0.16 \mathrm{M} \mathrm{KCl}$

$2.0 \mathrm{~g}$

$0.101 \mathrm{M} \mathrm{Na}_{2} \mathrm{HPO}_{4}$

$14.4 \mathrm{~g}$

$0.18 \mathrm{M} \mathrm{KH}_{2} \mathrm{PO}_{4}$

$2.4 \mathrm{~g}$

Dilute to $1 \mathrm{~L}$ with Mill-Q ddH $\mathrm{d}_{2} \mathrm{O}$

$\mathrm{pH}$ adjusted to 7.4

5 x Laemmli sample buffer $(100 \mathrm{~mL})$

4.0 M SDS

6.1 M Glycerol

$5.618 \mathrm{~g}$

$0.22 \mathrm{M}$ Tris- $\mathrm{HCl}$ (pH adjusted to 6.8)

$0.347 \mathrm{~g}$

$0.75 \mathrm{mM}$ Bromophenol blue

$5.024 \mathrm{mg}$

Dilute to $100 \mathrm{~mL}$ with Milli-Q ddH $\mathrm{d}_{2} \mathrm{O}$

$10 \% \beta$-mercaptoethanol by volume added immediately before use 
$10 \times$ SDS running buffer (1L)

SDS

$10.0 \mathrm{~g}$

Tris-base

$30.3 \mathrm{~g}$

Glycine

$144.1 \mathrm{~g}$

Dilute to $1 \mathrm{~L}$ with Milli-Q dd $\mathrm{H}_{2} \mathrm{O}$

Transfer buffer for Western blot (1L)

Tris-base

$3.03 \mathrm{~g}$

Glycine

$14.4 \mathrm{~g}$

$20 \%$ Methanol

$200 \mathrm{~mL}$

Dilute to $1 \mathrm{~L}$ with Milli-Q $\mathrm{ddH}_{2} \mathrm{O}$

Prepare fresh and chill to $4^{\circ} \mathrm{C}$ prior to use

5X TBE (Tris-borate EDTA) buffer (1L)

$450 \mathrm{mM}$ Tris

$54.0 \mathrm{~g}$

$450 \mathrm{mM}$ Boric acid

$27.5 \mathrm{~g}$

10 mM EDTA

$3.72 \mathrm{~g}$

Dilute to $1 \mathrm{~L}$ with Milli-Q ddH $\mathrm{H}_{2} \mathrm{O}$

TBS (1 L)

$50 \mathrm{mM}$ Tris

$6.0 \mathrm{~g}$

$150 \mathrm{mM} \mathrm{NaCl}$

$8.76 \mathrm{~g}$

Dilute to $1 \mathrm{~L}$ with Milli-Q ddH $\mathrm{H}_{2} \mathrm{O}$

$\mathrm{pH}$ to 7.5 with conc. $\mathrm{HCl}$

TBST (1 L)

Tween-20

$1.0 \mathrm{~mL}$ 
Dilute to $1 \mathrm{~L}$ with TBS 